\title{
Numerical Study of Lymph Mechanics
}

\author{
by \\ Daniel J. Watson
}

Submitted to Swansea University in fulfilment of the requirements for the Degree of Doctor of Philosophy

in the

Zienkiewicz Centre for Computational Engineering

College of Engineering

February 2021

Copyright: The author, Daniel J. Watson, 2021. 



\section{Acknowledgements}

The author would like to acknowledge the support and advice of his supervisor Dr.

Raoul van Loon as well as his second supervisor Prof. Kenith Meissner. The author would also like to thank Dr. Jason Carson and Dr. Igor Sazonov for their input and many fruitful conversations. The author also thanks his collaborators Prof. David

Zawieja, Prof. James E. Moore Jr., Kevork Karakashian and Cheryl Pike. 


\section{Declaration of Authorship}

This work has not previously been accepted in substance for any degree and is not being concurrently submitted in candidature for any degree.

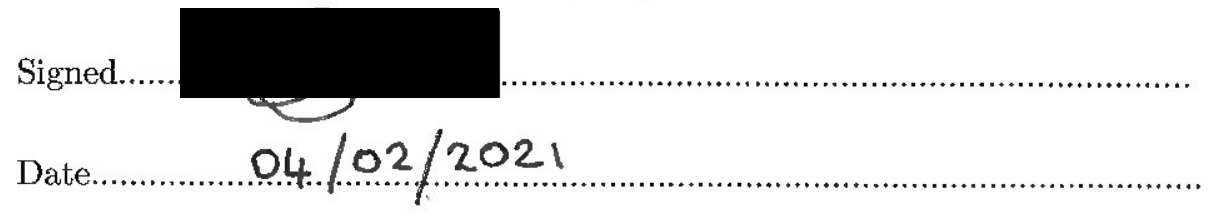

This thesis is the result of my own investigations, except where otherwise stated. Other sources are acknowledged by footnotes giving explicit references. A bibliography is appended.

Signed.......

Date $.04102 / 2021$

I hereby give consent for my thesis, if accepted, to be available for photocopying and for inter-library loan, and for the title and summary to be made available to outside organisations.

Date.........14.102/2021 


\section{Contents}

$\begin{array}{lll}\text { Acknowledgements } & \text { iii }\end{array}$

Declaration of Authorship iv

List of Figures $\quad$ ix

List of Tables $\quad$ Xv

Abbreviations $\quad$ xvii

1 Introduction. $\quad 3$

1.1 The Lymphatic System _ . . . . . . . . . . . . . . . . . . 3

1.1 .1 Lymph . . . . . . . . . . . . . . . . . . 4

1.1 .2 Lymphoid Organs _. . . . . . . . . . . . . . . . . 5

1.2 The Lymphatics of the Upper Limb and Their Impact in Breast Cancer

Related Lymphoedema. . . . . . . . . . . . . . . . . . . 6 6

1.2.1 The Initial Lymphatics and the Lymphatic Capillaries. . . . . . . . 7

1.2 .2 The Collecting Lymphatics . . . . . . . . . . . . . . . . 8

1.2.3 The Lymph Nodes of the Upper Limb . . . . . . . . . . . . 8

1.2.3.1 Axillary Nodes . . . . . . . . . . . . . . . . . . 8

1.3 Biomechanics of Lymphangions . . . . . . . . . . . . . . . . . . . . . . . . . . 9

1.3.1 Behaviour of the Wall . . . . . . . . . . . . . . . . 10

1.3 .2 Behaviour of the Valve . . . . . . . . . . . . . . . . . 12

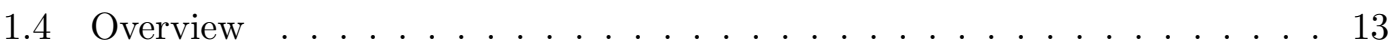

2 Modelling a Single Lymphangion: 3D FEA.

2.1 Introduction . . . . . . . . . . . . . . . . . . . . . . 15

2.2 Methods . . . . . . . . . . . . . . . . . . . . . 17

2.2.1 Image Processing and Segmentation . . . . . . . . . . . . 17

2.2.1.1 Segmentation and Reconstruction . . . . . . . . 18

2.2.2 Material Properties of the Wall and Leaflets. . . . . . . . . . . . 20

2.2 .3 FEA Model Setup . . . . . . . . . . . . . . . . . . . . 23 
2.3 Results . . . . . . . . . . . . . . . . . . . . . 24

2.4 Discussion . . . . . . . . . . . . . . . . . . . 27

3 Developing a 0D Lumped Model for the Lymphatic System: From Lymphangion to Limb. $\quad \mathbf{2 9}$

3.1 Modelling Flow . . . . . . . . . . . . . . . . . . . 31

3.2 Lymphangion Valves . . . . . . . . . . . . . . . . . . . . . . 33

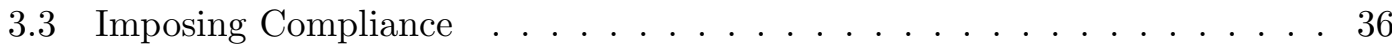

3.4 Imposing Contraction . . . . . . . . . . . . . . . . . . . . 39

3.4.1 Modulating Tension . . . . . . . . . . . . . . . . . . . . . 39

3.4.2 Refined Modulating Stiffness Model . . . . . . . . . . . . . . . 40

3.4.3 Increasing Contraction Frequency with Trans-mural Pressure. . . . 43

3.4.4 Discretisation and Assembling a Single Lymphangion . . . . . . . . 46

3.5 Assembling From a Vessel to a Lymphatic Network for the Upper Limb . 51

3.5.1 A Lymphatic Network for the Upper Limb . . . . . . . . . . . . . 52

3.6 Metrics for Exploring Lymphatic Function . . . . . . . . . . . . . . . . . . . . . 55

3.7 Convergence Study and Scheme Stability . . . . . . . . . . . . . . 57

4 Exploring a 0D Model of the Lymphatic System: From Lymphangion to Limb. $\quad 61$

4.1 Introduction . . . . . . . . . . . . . . . . . 61

4.2 Methods . . . . . . . . . . . . . . . . . . 62

4.2 .1 Sensitivity Analysis . . . . . . . . . . . . . . . 62 62

4.2 .2 Parameter Estimation . . . . . . . . . . . . . . . . . . 63

4.2 .3 Lymphangion Chain Study. . . . . . . . . . . . . . . . . . 65

4.2 .4 Limb Network Study. . . . . . . . . . . . . . . . . . . 66

4.3 Results. . . . . . . . . . . . . . . . . . . 67

4.3.1 Sensitivity Analysis . . . . . . . . . . . . . . 6 67 . . . . . 67

4.3.2 Coordination of Lymphatic Pumping . . . . . . . . . . . . . 68

4.3 .3 The Effects of Inertia . . . . . . . . . . . . . . . . . . 72

4.3.4 Exploring the Effects of Pressure Loading . . . . . . . . . . . . . 74

4.4 Discussion . . . . . . . . . . . . . . . . . . . . 77

4.4.1 Network Scale Model . . . . . . . . . . . . . . . . . . . . . 77

4.4.1.1 Parameter Set . . . . . . . . . . . . . . . . . . . . . . . . 77

4.4.1.2 The Limb Network . . . . . . . . . . . . . . . . . 77

4.4.1.3 A Regulatory Mechanism . . . . . . . . . . . . . 78

4.4.2 Discrepancies Between the Single Lymphangion Model and Previously Published Experimental Data. . . . . . . . . . . . . . . . 79

4.4.2.1 A: Pressure Curves . . . . . . . . . . . . . . . . 79

4.4 .2 .2 B: Insufficient Contraction . . . . . . . . . . . . . 80

4.4.2.3 C: Slope of Contraction . . . . . . . . . . . . . . 80

4.4.2.4 D: Relaxation Slope . . . . . . . . . . . . . . . . . 81

4.4.2.5 E: Loss of Volume . . . . . . . . . . . . . . . 81 
4.4.2.6 F: Lack of Retrograde Fluid Motion . . . . . . . . . . 82

4.4.2.7 G,H: Refractory Period . . . . . . . . . . . 82

4.5 Conclusion . . . . . . . . . . . . . . . . . . . 84

5 Breast Cancer Related Lymphoedema and its Effect on Limb Shape. 87

5.1 Introduction . . . . . . . . . . . . . . . 87

5.1 .1 The Diagnosis of Lymphoedema . . . . . . . . . . . . . . 88

5.1.1.1 Consequences of an Earlier Diagnosis . . . . . . . . . . 94

5.2 Developing a New Diagnostic Method . . . . . . . . . . . . . . . . 94

5.3 Methods . . . . . . . . . . . . . . . . . . . 95

5.3 .1 Manifold Harmonic Analysis . . . . . . . . . . . . . . . . . 96

5.3 .2 The Laplace-Beltrami Operator . . . . . . . . . . . . . . . . . . . . . . . . . . . . . . . . . . .

5.3 .3 Global Point Signature . . . . . . . . . . . . . . . . . . 98

5.3 .4 G2-distribution Metrics . . . . . . . . . . . . . . . . . . 100

5.3.4.1 Entropy and Redundancy . . . . . . . . . . . . . . . 101

5.3 .4 Noise . . . . . . . . . . . . . . . . . . 102

5.3 .5 Finite Element Analysis . . . . . . . . . . . . . . . 103

5.4 Results . . . . . . . . . . . . . . . . . . . . 105

5.4 .1 In-silico Study . . . . . . . . . . . . . . . . . 105

5.4.1.1 Sensitivity to Noise . . . . . . . . . . . . . . . . . 105

5.4.1.2 Pressurisation . . . . . . . . . . . . 106

5.4 .2 In-vivo Study . . . . . . . . . . . . . . . . . . . 106

5.5 Discussion . . . . . . . . . . . . . . . . . . . . . 109

5.6 Conclusion . . . . . . . . . . . . . . . . . . 110

6 Concluding Remarks $\quad 113$

A Kálmán Filter Overview

$\begin{array}{ll}\text { B Contraction Function Derivation } & 119\end{array}$

$\begin{array}{lr}\text { C Upper Limb Network } & 121\end{array}$

$\begin{array}{ll}\text { Bibliography } & 137\end{array}$ 



\section{List of Figures}

1.1 An overview of the lymphatics of the upper limb. A single vessel is traced from the hand to the shoulder. Bottom: The initial lymphatics with button-junctioned endothelial cells forming oak-leaf shaped single leaflet valves in the wall of the vessels. Middle: Collecting lymphatics with semilunar leaflet valves to prevent fluid from flowing back toward the tissues. Top: a supratrochlear lymph node with its afferent sub-capsular sinus. . .

2.1 An overview of the lymphatic valve configuration and nomenclature. A) Nomenclature for the valve leaflets. B) The configuration of the leaflets to form the valve. C) Shows the Segmented structures of a subject-specific valve. Leaflets are shown in red and green, and the wall is shown in blue.

2.2 A series of z-y slices illustrating a step by step overview of the segmentation process. A) A z-y image slice. B) A segmentation of the wall (yellow). C) The guidelines of leaflet edges used in reconstruction (cyan). D) Manual reconstruction of the leaflet structures (red and blue). . . . . 20

2.3 A plot of the mean $D / D_{0}$ for ten equally spaced bands taken axially along the wall geometry during pressurisation. Colour indicates the location of the bands, shown on the lymphangion geometry in the sub-figure. The black line represents the pressure diameter relationship found by Rahbar et al. [1].

2.4 A plot of the peak axial displacement of the trailing edge against transvalvular pressure. Also shown is the original valve at three different trans-valvular pressures with the Euclidean displacement field coloured. A marks the first contact between the leaflets. B marks the point of minimal orifice area and $\mathrm{C}$ is the maximum applied trans-valvular pressure.

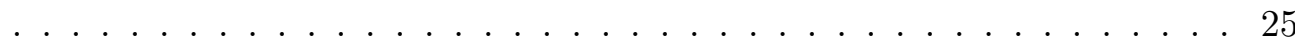

2.5 Trans-valvular pressure required to close the valve for differing leaflet shear moduli. The black lines represent experimental data for a lymphatic valve without tone and a trans-mural pressure of $5 \mathrm{cmH}_{2} \mathrm{O}[2] \ldots 26$

2.6 Retrograde volume displaced for differing leaflet shear moduli. . . . . . . 27

3.1 A circuit equivalent overview of a lymphangion . . . . . . . . . . 31 
3.2 A) The trans-valvular pressure required to close the valves at differing trans-mural pressures, reproduced from Davis et al. [2]. The black line indicates a linear fit with the equation shown. B) Shows a trans-valvular pressure - normalised resistance plot for the valve demonstration shown in C. The line colour shows the time allowing the increase in valve open bias present at higher pressures. The hysteresis can be seen which appears diminished at higher pressures. Shown, in black is the relationship in the Bertram et al. model without hysteresis or trans-mural pressure dependent biasing. C) Shows a sinusoidal trans-valvular pressure with an increasing trans-mural pressure. Markers indicate a change in valve states for the Bertram et al. and proposed model, found by identifying when the normalised resistance crosses a $50 \%$ threshold . . . . . . . . 36

3.3 The passive pressure diameter relationship proposed by Rahbar decomposed into the following functions [1]. A strain-stiffening function at high stretches, $P_{\text {Strain-Stiffening }}^{*}$, shown in blue. A strain-thinning function incipient to collapse, $P_{\text {Strain-Thinning, }}^{*}$, shown in magenta. $P_{\text {Residual }}^{*}$ the residual with the above functions removed, shown in green. Finally, the sum of the above three functions is shown in black. Left axis is the normalised pressure ratio. Right axis is the pressure if $P_{d}$ is 735 dynes $\mathrm{cm}^{-2} \ldots \ldots$.

3.4 The model defined in Section 3.4.4 is used to illustrate the effects of changing the contraction parameters against previously published experimental data. Apart from the parameters under investigation the coefficients are as stated in 4.2. Simulated diameters are shown in colour. Dashed line represents a contraction from Davis et al. [2]. . . . . . . . . . . .

3.5 The constitutive law in Section 3.3 is used to rescale McHale's pressurefrequency data into pressure-stretch data[3]. Through which a piecewise linear approximation is fitted. . . . . . . . . . . . . . . . 44

3.6 Top: the normalised diameter $D / D_{d}$ is shown for a lymphangion pumping against an increasingly adverse pressure gradient. Bottom: the corresponding frequency $\bar{\omega}$ for increasing values of $K_{\omega} \ldots \ldots \ldots$. . . . . . 45

3.7 The simplest representation of a lymphangion as a Hagen-Poiseuille resistance between two pressures . . . . . . . . . . . . . . 46

3.8 A representation of a lymphangion with a compliance representing the walls 47

3.9 An equivalent circuit diagram for a lymphangion including a valve . . . . 49

3.10 The complete equivalent circuit diagram for a lymphangion including inertia 50

3.11 The equivalent circuit diagram for a vessel of a single lymphangion. Note the addition of valve block at the left hand side. . . . . . . . . . . . 52

3.12 A vessel is extended through the addition of more lymphangion elements. Note the lumping of elements leaves one pressure node per lymphangion as discussed in section 3.4.4 . . . . . . . . . . . . . . 5 52

3.13 A: Planche III from Sappey is shown with the traced vessels overlaid in green [4]. B: The reconstructed network is shown after the traced sections were projected onto a series of fustra $\ldots \ldots \ldots \ldots \ldots$ 
3.14 A: The final lymphatic limb network is presented in cylindrical form with the axilla located at the bottom of the image and the hand at the top. B: The distribution of vessel diameters in the lymphatic limb network. C: the distribution of vessel connectivities in the lymphatic limb network. . . 55

3.15 Plots of lymphangion volume, pressure, flow rate and the upstream, $\zeta_{u}$ and downstream $\zeta_{d}$ valve state are shown with a single contraction cycle in bold. . . . . . . . . . . . . . . . . . . . . 5 57

3.16 The temporal convergence is shown for each of the solution variables and

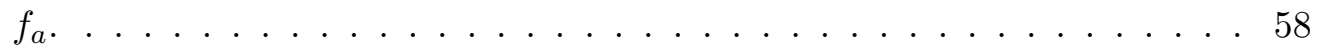

3.17 The flow rate, diameter, pressure and upstream valve state are shown for a "fluttering" lymphangion. The red line indicates without an inertial term and the blue line with an inertial term. Note the relatively high consequences of flutter on the flow rate and diameter but small consequences on the pressure. . . . . . . . . . . . . . . . . . . . . . 59

4.1 A cylindrical representation of the limb network model with the boundaries marked. . . . . . . . . . . . . . . . . 66 66

4.2 Left: the first order and total sensitivity index of each parameter on average flow rate, $\bar{Q}_{c}$, is shown with the $95 \%$ confidence interval. Right: the first order and total sensitivity index of each parameter on pumping efficiency, $\eta_{\text {pump }}$, is shown with the $95 \%$ confidence interval. . . . . . . 68

4.3 The contraction state, $\hat{M}_{t}$, of a chain of 10 lymphangions pumping against a $1 \mathrm{cmH}_{2} \mathrm{O}$ pressure difference is shown at four different time points over 10 minutes of simulation. Coloured by distance from the inlet, blue, to the outlet, red. . . . . . . . . . . . . . . . . . 69 69

4.4 Four cylindrical representations of the lymphatic limb network coloured from blue to yellow proportional to the contraction state $\hat{M}_{t}$ showing the initialisation of contractions against a $1 \mathrm{cmH}_{2} \mathrm{O}$ pressure difference. . . . . 69

4.5 The probability density of contraction duration of a lymphatic limb network pumping against a $1 \mathrm{cmH}_{2} \mathrm{O}$ pressure difference over 600 seconds. $\quad 70$

4.6 A radial representation of the limb network is shown coloured by the final contraction duration of 600 second simulation pumping against a 1 $\mathrm{cmH}_{2} \mathrm{O}$ pressure difference . . . . . . . . . . . . . 71

4.7 A radial representation of the limb network is shown coloured by the average $D / D_{0}$ ratio for the final contraction cycle of a 600 second simulation pumping against a $1 \mathrm{cmH}_{2} \mathrm{O}$ pressure difference . . . . . . . . . . 72

4.8 Pressure and diameter traces showing the consequences of omitting the inertial term is shown for a single lymphangion after 200 seconds of simulation. Also shown is the average absolute relative error in both diameter and pressure over the entire simulation period . . . . . . . . 73

4.9 The relative error in the average flow rate, $\bar{Q}_{c}$, is shown for differing pressure conditions. . . . . . . . . . . . . . . . 74

4.10 The effects of different pressure states on the average flow rate, mean $\bar{Q}_{c}$. Vertical bars indicate the standard distribution of $\bar{Q}_{c} \ldots \ldots 75$ 
4.11 Violin plots of the distribution of $\bar{Q}_{c}$ is shown for the network model with differing pressure boundary conditions. The mean of $\bar{Q}_{c}$ is also shown in blue. . . . . . . . . . . . . . . . . . 76

4.12 Violin plot of the distribution of the pumping efficiency, $\eta_{c}$, for differing pressure boundary conditions. . . . . . . . . . . . . 76

4.13 Left: the frequency response found by Seki et al. [5]. Right: Experimental data[2] is presented along with the fit shown from Figure 4.13 also shown is the filtered simulation result using a filter defined by the frequency response shown on the left. . . . . . . . . . . . . . . 8 80

4.14 A comparison of the model fitted against previously published experimental results. In the experimental data, a lymphangion was presented with an initial upstream and downstream pressure of $1 \mathrm{cmH}_{2} \mathrm{O}$. After the seventh contraction the downstream pressure was increased at a rate of $4 \mathrm{~cm} \mathrm{H}_{2} \mathrm{Omin}^{-1}$ until the downstream pressure was $8 \mathrm{cmH}_{2} \mathrm{O}$ at which point the upstream pressure was returned rapidly to $1 \mathrm{cmH}_{2} \mathrm{O}$ [2]. This process is repeated for the single lymphangion model. Key discrepancies between the simulated and experimental data are highlighted and annotated. These are discussed in section 4.4.2. . . . . . . . . . . . . .

5.1 A diagram explaining the operating principle of a perometer. A frame containing two perpendicular arrays of infra-red LEDs opposed by sensors are moved with both arrays orthogonal to the arm. The cross-section is assumed to be a ellipse, with the semi-major and minor axis having the same lengths as the shadows cast by the limb. . . . . . . . . . .

5.2 A flowchart showing the study design considering upper limb scans for group of 24 lymphoedema patients. In an in-silico study, three patients of this group were used to develop a shape-based metric that was then used in a statistical shape analysis of the remaining 21 patients. . . . . . . 96

5.3 A figure demonstrating the Meyer et al. construction of the LaplaceBeltrami operator [6]. For a node, $i$, the area weight, $A_{i i}$, is the Voronoi area associated with the node. For each associated edge $(i, j)$ the weight $w_{i j}$ is found as a function of the two corners that oppose $i j, \beta_{i j}$. For the leading diagonal of $\mathbf{W}$ the values are the negation of the sum of every weight associated with that node. . . . . . . . . . . . 98

5.4 The first, sixth, eleventh and sixteenth eigenvectors of the Laplace-Beltrami operator are shown for the case one limb geometry. Colour is normalised eigenvector coffecient. . . . . . . . . . . . . . .

5.5 A plot showing the power-eigenvalue number relationship for the three insilico geometries. The solid lines represent least-squares fits of a 'dog-leg' function with the inflection point representing the point of noise dominance. The eigenvalue numbers are such that the Fiedler vector would have an eigenvalue number of 1 and the highest frequency would be numbered $n$. . . . . . . . . . . . . . . . . . . . . . . . . . . . . . . 103 
5.6 Relative error in G2-entropy and volume for each of the unaffected geometries removed from the original study with the presence of an additive (top) and multiplicative (bottom) Gaussian noise. The bars represent one standard deviation for 100 runs. . . . . . . . . . . . . . . . . . . 105

5.7 In-silico simulations illustrate the effect of limb swelling on the G2-distributions for GPS series that are truncated from the Fiedler vector to the point of noise dominance. The colours represent the hydrostatic pressure in $\mathrm{kPa}$. . 107

5.8 Redundancy change associated with pressurisation for each in-silico case. Also shown are cross-sections of case one for three applied loads. . . . . 107

5.9 Violin plots for affected and unaffected limbs. Top: G2-redundancy. Bottom: volume. A significant difference exists for both. On the G2redundancy plot a hypothetical threshold of $7.05 \%$ is shown as a horizontal dashed line. . . . . . . . . . . . . . . . . . . . . . . 108

5.10 The signed difference between the affected and unaffected arm in G2redundancy and volume is plotted for each patient. Difference $=$ AffectedUnaffected. No statistically significant relationship was found between the two. . . . . . . . . . . . . . . . . . . . . 108 



\section{List of Tables}

4.1 A table showing the range of parameters used in the sensitivity analysis. . 63

4.2 A table of the values found by manually fitting the model against the data from Davis et al.[2] . . . . . . . . . . . . . . . . 6 65

5.1 A table of diagnostic criteria used in clinical studies of BCRL and their reported incidence. $\dagger$ from Cheville et al.[7]. $\ddagger$ Only ALND patients are produced from a study which included non-ALND patients. Non-axilla targeted radiation is not considered though some patients received it. Chemotherapy was given to most but not all patients. Modified radical mastectomies are omitted, stated mastectomies are simple or segmented . 92

5.2 A table showing the CTCv3 grading for lymphoedema as defined in [7] . . 93

5.3 Published diagnostic sensitivities and specificities for upper-limb breast cancer related lymphoedema $[8] \ldots 110$ 



\section{Abbreviations}

BRCL Breast Cancer Related Lymphoedema

FEA Finite Element Analysis

MHA Manifold Harmonic Analysis

MHT Manifold Harmonic Transform

CFD Computational Fluid Dynamics

ODE Ordinary Differential Equations

PDE Partial Differential Equations

FIR Finite Impusle Response

ICG IdoCyanine Green

NIRF Near Infra-Red Fluorescence

LVA LymphaticoVenular Anastomosis

FSI Fluid Structure Interaction 

Dedicated in Loving Memory Jayne Watson

December 1962 - October 2016 

SWANSEA UNIVERSITY

\author{
Summary \\ Zienkiewicz Centre for Computational Engineering \\ College of Engineering \\ Doctor of Philosophy \\ by Daniel J. Watson
}

Methods taken from engineering and computer science were applied to the lymphatic system. Starting with a 3D analysis of a single subject-specific lymphatic valve. A mechanism was presented to explain previous experimental results showing the effect of trans-mural pressure on the pressure required to close lymphatic valves. The importance of wall motion in future FSI studies of lymphatic valve dynamics were identified. Previous approaches to lumped modelling of the lymphatic system were considered and modifications were proposed. A less-idealised valve model, incorporating trans-mural dependent bias, was proposed as well as a method of allowing self-organised contraction through a stretch-dependent frequency of contraction. A network of the superficial lymphatics of the upper-limb was reconstructed from an anatomical sketch. The network was used in conjunction with the lumped model to produce a 421 vessel lymphatic model consisting of 17,706 lymphangions. Several issues which impede large network scale modelling of the lymphatic system are identified. A simplified patient-specific biphasic model of lymphoedema was proposed and used to develop a novel shape-based metric for lymphoedema. A statistically significant relationship between the metric and the presence of lymphoedema was found. 



\section{Chapter 1}

\section{Introduction.}

In the field of biomechanics, the lymphatic system has not received the attention that has been afforded the arterial and vascular systems. As a result the physical mechanisms of many lymphatic diseases are poorly understood, as is the reasoning behind diagnostic and therapeutic interventions. This work builds on previous attempts to model fluid flows within the lymphatic system with the aim of providing a more holistic understanding of the mechanisms behind lymphatic insufficiency.

\subsection{The Lymphatic System}

Lymphatic insufficiency causes the tissues to swell with an accumulation of lymph the fluid transported by the lymphatic system. This work is an attempt to numerically model the transport of lymph. In order to begin it first necessary to consider a definition of lymph and by extension the lymphatic system. It is the difficulty encountered in these definitions that provides much of the interest and complexity to this undertaking. 


\subsubsection{Lymph}

The lymphatic system is often considered in relation to the cardiovascular system. This relationship is not without support. The lymphatic system emerges from the cardiovascular system during embryonic development [9]. Like blood in the cardiovascular system; a fluid, lymph, is transported through vessels and adapted by organs. However, two differences between the cardiovascular and lymphatic system complicate this understanding.

Firstly, the lymphatic system is not a circulatory system, it is directed, with fluid ultimately moving from the interstitia to the thoracic duct [10]. Unlike capillaries within the cardiovascular system, initial lymphatics rely on single leaflet valves within the lymphatic wall to draw fluid directly and solely from the interstitia. This fluid is then transported through larger collecting lymphatics, draining into the thoracic duct to sit in the cisterna chyli before draining into the vascular system.

During this process the constituents of the lymph change: lymph nodes present the opportunity for communication between lymph and blood across a semi-permeable barrier. Unlike the cardiovascular system, in which blood is mixed and recirculated giving it a systemic quality, the constituents of lymph at any point within the lymphatic system are dependent on their origin and journey [11]. In health, this can be seen in the mesenteric lymph which gathers fats from chyle in the intestines and transports it to the liver where they are exchanged for hepatic proteins, giving this lymph a unique constitution. In disease this can be observed in the presence of inflammatory factors in tumour sampling lymphatics [12]. This concept can and has been extended to vaccination, infection and metastasis [13][14]. The extracellular fluid around a local pathology could contain a mixture of cytokines, immune cells, and malignant tumour cells that are swept into the lymphatic system. Once in the lymph, they mix in varying proportions producing spacial gradients and discontinuities across the lymphatic system. The spatial variation of these species effects the recruitment of immune cells, the severity of an immune response and the location of metastasis. The directed nature of the lymphatic system could be exploited to develop diagnostic techniques and therapies. 
However, these methods would be dependent on understanding the flows which develop throughout the lymphatic system.

Secondly, the lymphatic system in mammals has no central pump unlike the heart in the analogous cardiovascular system. Rather pumping is distributed along collecting lymphatics which draw from the initial lymphatics that sample the interstitia [15]. Semi-lunar check valves along the length of the collecting lymphatic vessels demarcate lymphangions, which contract to propel fluid along the vessel. Whilst this property complicates the modelling of lymph, which will be discussed in later sections; it does allow for a phenotypical definition that will used heavily throughout the remainder of this work. Collecting lymphatic vessels consist of a series of contractile elements, lymphangions, which are separated by lymphatic valves.

\subsubsection{Lymphoid Organs}

The primary lymphoid organs are bone marrow and the thymus; while these have large immunological impact, they have little to no impact on the flows within lymphatic system. The secondary lymphoid organs consist of the spleen and lymph nodes. It is these lymph nodes which have a profound impact on the flows of the lymphatic system. It is often their removal during the course of cancer treatment which leads to secondary lymphoedema. Tertiary lymphatic organs such as gastric associated lymphatic tissue, Peyer's patches, and arterial tertiary lymphoid organs have important immunological roles to play but are considered of little importance in the understanding of lymphatic flow.

The lymph nodes filter lymph from collecting lymphatics allowing immunological sampling and dendritic cell T-cell interaction. They also place lymph and blood either side of a semi-permeable structure, allowing communication between the two. Not only does this allow the dendritic cells to return to systemic blood [16], it also allows the diffusion of small molecules. A large quantity of lymph is allowed to bypass the majority of the densely packed cells of the cortex by passing through a sub-capsular sinus. The 
complex and multifaceted behaviour of the lymphoid organs is beyond the scope of this work.

\subsection{The Lymphatics of the Upper Limb and Their Impact in Breast Cancer Related Lymphoedema.}

In lymphoedema the lymphatic system removes insufficient fluid from interstitia to prevent its accumulation, leading to a swelling of the tissues. The cause of this can be genetic in origin leading to primary lymphoedema or it can be caused by an injury to lymphatic system leading to secondary lymphoedema. The treatment of breast cancers often requires the removal of several nodes that receive lymph drained from the upper-limb; somewhere between 17 and $54 \%$ of these patients will develop secondary lymphoedema [7].

This work will focus on secondary lymphoedema of the upper limb in the context of breast cancer. In this section the anatomy of the lymphatic system within the upper limb is presented along with its relevance to understanding the condition. Figure 1.1 shows a single vessel traced from the hand to the shoulder with the differing microstructures of the lymphatic system shown. 


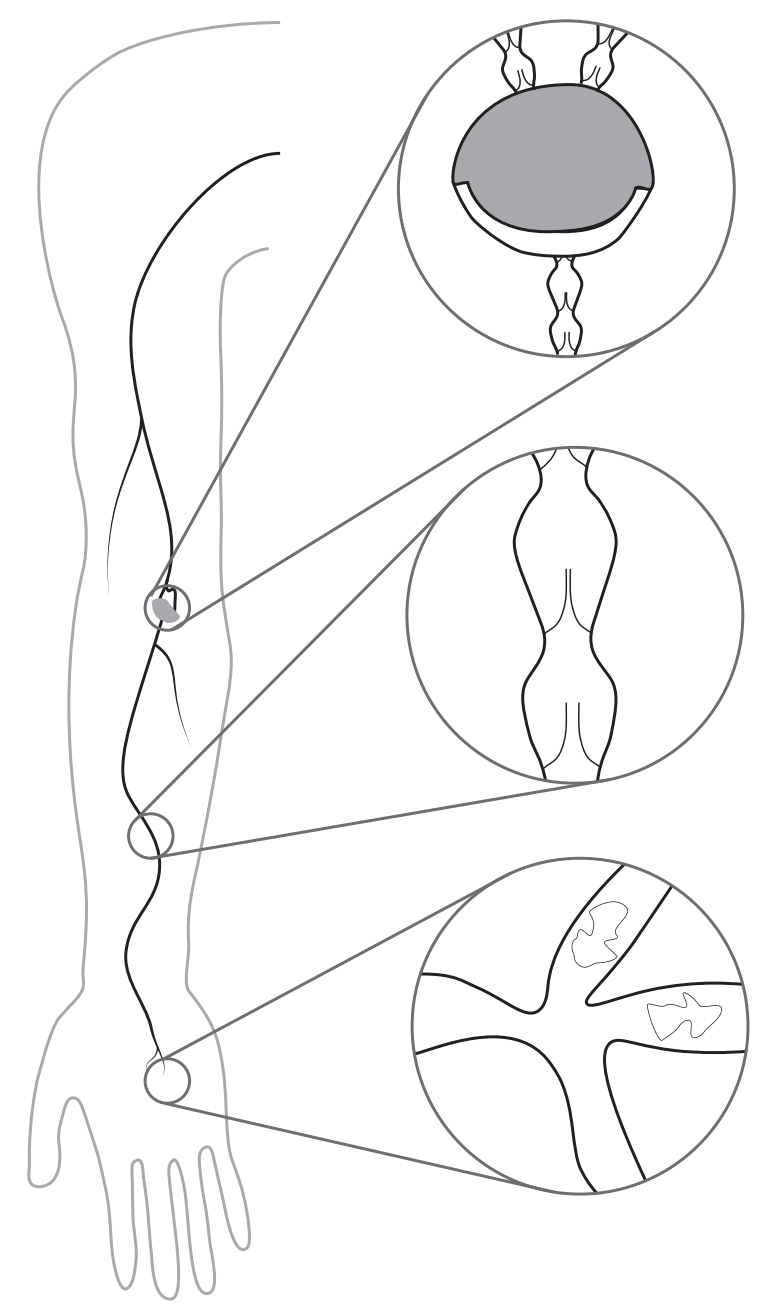

Figure 1.1: An overview of the lymphatics of the upper limb. A single vessel is traced from the hand to the shoulder. Bottom: The initial lymphatics with buttonjunctioned endothelial cells forming oak-leaf shaped single leaflet valves in the wall of the vessels. Middle: Collecting lymphatics with semi-lunar leaflet valves to prevent fluid from flowing back toward the tissues. Top: a supratrochlear lymph node with its afferent sub-capsular sinus.

\subsubsection{The Initial Lymphatics and the Lymphatic Capillaries.}

Along with the vascular capillaries, the tissues also contain lymphatic capillaries. These valveless structures draw interstitial fluid and represent the start of the lymphatic system. The lymphatic capillaries are interconnected independent of other lymphatic structures and can be used to allow lymph to flow "reflux" around an injury to the lymphatic 
system, albeit with increased resistance. The capillary bed is drained by pre-collecting lymphatics which are contractile but lack valves [13].

\subsubsection{The Collecting Lymphatics}

The collecting lymphatics carry lymph from the initial lymphatics to and between lymph nodes, until it reaches the cisterna chyli at the spine, where lymph resides until it enters the lymphatic ducts and is returned to the vascular system. The collecting lymphatics are commonly divided into two groups; the deep lymphatics, which drain primarily from the muscles and superficial lymphatics, which drain from the cutaneous tissues [17].

\subsubsection{The Lymph Nodes of the Upper Limb}

There are usually only a few lymph nodes within the limb itself. The supratrochlear sometimes called epitrochlear - glands which consist of one to four nodes present on the medial aspect of the elbow and one or two deltopectoral - sometimes called infraclavical - nodes present between the deltoid and pectoral muscle [18][19]. After leaving the limb the majority of lymph drains into the axillary.

\subsubsection{Axillary Nodes}

Breast cancer related lymphoedema, BCRL, is usually the result of injury to the lymph nodes of the axilla. The axillary lymph nodes are 20-49 nodes which integrate lymph flows from the arm with flows from the pectoral, breast and trunk [17]. These nodes have a longitudinal diameter between 5.4 and $23 \mathrm{~mm}(\mathrm{M}=14.82 \mathrm{~mm}, \mathrm{~S} . \mathrm{D}=3.45 \mathrm{~mm})$ and a transverse diameter of between 3 and $12 \mathrm{~mm}(\mathrm{M}=6.88$, S.D $=3.45 \mathrm{~mm})[20]$. In the majority of patients the lymphatics of the upper limb all flow into nodes in the axillary group. However, cases have been reported where drainage from some regions of the arm bypassed the axillary group and drained to the trunk or cervical nodes [19]. There is 
also evidence of pre-nodal lymphovenous communication within the arm of pre-operative axilla lymphandectomy breast cancer patients [21].

\subsection{Biomechanics of Lymphangions}

The anatomical study of the lymphatic system has its roots in several misinformed descriptions of the lymphatic system beginning in 500BC. However, it wasn't until the end of the Renaissance that the understanding was in place to allow considerations of the biomechanics of the lymphatic system [22]. There are two aspects of lymphangions which motivate the study of their biomechanics: the contractile and the valved nature of lymphangions. Sappey identified lymphatic valves in the vessels of the upper limb in 1855 and noted that they had first been observed by Rudbeck in 1653 [23]. Sappey concluded that lymphatic valves gave lymphatic vessels a directionality. By 1895, Ranvier was hypothesising the embryonic formation of lymphatic valves [24][25].

Unlike the lymphatic valves, the contractile nature of lymphangions went unreported until the 18th century. The discovery of the contractility of lymphatics is complicated by the simultaneous discoveries made by authors unaware of each other, Aukland's 2005 review of these works makes clear the progression of research [26]. As Aukland notes Hewson hypothesised in 1774 that lymph could be drawn into the lymphatic vessels by capillary forces and then propelled by contraction of the lymphangions. In 1784, Sheldon noted that the cervical lymphatic vessel walls were muscular in dogs. He hypothesised that the musculature, along with the motion of respiration and the motion of the heart, propelled lymph forward [27]. Hewson and Sheldon's theory was confirmed by Heller in 1896, without prior knowledge of those hypotheses [26]. Heller observed the contractility of mesenteric lymphatics in guinea pigs. In 1926, Carrier observed contractions in the lymphatic vessels of bats wings [26]. However, Carrier didn't appear to be aware of the nature this discovery and considered the contractility of lymphangions to be well known. Florey reported the observation of the contractility of mesenteric lymphangions in 1927, aware of Heller's work via Tigerstedt but unaware 
of Carrier, Sheldon, or Newson[23][26]. Florey also recorded observations of the valves moving with the peristalsis of the gut and suggested they were driven by differences in pressure [23]. The contractility of pre-nodal lymphangions of the extremities was first recorded in 1949 by Smith without reference to Carrier [28]. While Zhdanov's discovery of the lymphatic duct in 1949 completed the modern understanding of the organ-scale anatomy of the lymphatic system [29][10]; it would be another twenty years before a series of works began to explore the mechanisms and function of these behaviours and lymphatics entered the field of biomechanics.

\subsubsection{Behaviour of the Wall}

In 1973 Mawhinney and Roddie studied the spontaneous contraction of the bovine mesenteric lymphatics [30]. They constructed an apparatus which held a section of lymphangion submerged in a buffered solution and connected to a transducer. They recorded the axial force of contraction, and the frequency of contraction under the influence of several drugs. In 1975, McHale and Roddie expanded this apparatus to cannulate both ends of the vessel allowing not only pressure measurements just downstream of the lymphangion but also allowing differing trans-mural and trans-valvular pressure conditions to be imposed by adjusting the height of fluid reservoirs for the afferent and efferent cannula. This allowed the study of not only the effect of trans-mural pressure on bovine lymphatic vessels but also provided data on stroke volume, contraction frequency and flow rate [3]. This design of apparatus has been used in modern works with the only changes being improved instrumentation [2]. These techniques are used in the first study of the biomechanics of the lymphangion by Ohhashi et al. in 1980 [31]. Ohhashi et al. recorded the pressure-radius relationship for many lymphangions. They estimated the active components by comparing systole to diasystole, though this method would not account for the tone generated by the lymphatic muscle. They also estimated the Youngs' modulus from the pressure-radius relationship, making this the first attempt to find a constitutive law for the lymphatic vessel wall. For the purposes of this work we can split the remaining development of a material model into two groups. Firstly, 
works which attempt to understand the underlying biomechanics and advance these to a model which agrees well with experimental results. Secondly, works that attempt to produce functions which only recapitulate the experimentally observed pressure-radius or pressure-diameter relationships. Of these two tasks the second is simpler while the first is more informative. In the second case the range of functions to which experimental data can be fitted is infinite, while in the first case the range of material models with relevant microstructure is limited. The second case allows an understanding of why the material behaves as observed experimentally, this allows the model to be adjusted for inter-species variation in microstructure and also explains the material properties in situations outside of those observed i.e. uniaxial loading. This issue will be discussed in greater detail in Chapters 3 and 4.

For the first case, Reddy's model of the lymphatic system in 1979 was based on Hookean thin wall cylinder with an additional stress term to represent the contraction [32]. A similar method was used by Quick et al. but with a time-varying elastance used to impose contraction [33]. This tube-law approach continued with MacDonald et al. in 2008, adding viscous damping and longitudinal bending to this approach [34]. As MacDonald et al. noted, their experimentally found values for the modulus agreed with previous values found in thoracic ducts at low pressures but underestimated values found in bovine mesenteric lymphatics at higher pressures. This suggests that this model could not capture the highly non-linear shear-stiffening behaviour observed in lymphangions. This behaviour is believed to be caused by an elastin dominated response at low strains which transitions to a collagen dominated regime as initially wavy collagen fibres are pulled taut; in a similar mechanism to that found in arterial mechanics [34]. Contarino and Torro further modified this tube-law into a power law by raising each of the terms to a non-integer power [35]. Attempts at a microstructure motivated nonlinear constitutive law have also occurred. By using second harmonic generation for collagen and multiphoton excitation for elastin, Arkill et al. was able to achieve images of the elastin and collagen networks within bovine mesenteric lymphangions at differing strain states [36]. However, the authors didn't propose a strain - stress relationship or similar. Caulk et al. attempted to fit a four-fibre family model to experiments conducted 
on the thoracic duct of rats. However, as the author noted they struggled to recapitulate experimental data [37]. The use of thoracic ducts in the construction of lymphatic wall material models is motivated by their larger size. However, in a 1987 study Ohhashi found considerable site to site variation in the stiffness of lymphangions in canines, with a large difference between mesenteric lymphangions and the thoracic duct [38].

Attempts in the second case predominantly proceed from a 2012 study by Rahbar et al. [39]. As well as making qualitative statements of the composition of the wall and leaflet, in a manner similar to Arkill et al. [36]. The authors also recorded the pressure-diameter relationship for mesenteric rat lymphangions, in a manner similar to Ohhashi [31]. However, instead of calculating an equivalent initial Young's modulus the authors fitted an empirical relationship between the diameter and the pressure. This form was used in the lymphangion model proposed in 2014 by Bertram et al. [40]. With occasional modification and simplification it has been used in many subsequent works [41][42][43]. It is the only material model that has been used to predict and explain a phenomena that was subsequently demonstrated experimentally; the mechanism for sub-atmospheric lymphatic pumping [44]. It is also the material model that has been used at the greatest scale in terms of number of lymphangions [43].

Throughout the remainder of this work the approach to modelling the wall will be focused on the second case. The reliance on empirically derived relationships that do not attempt to model the underlying material behaviours that drive those relationships is a limitation of this work. However, these functions conveniently allow the capture of the highly non-linear wall behaviour.

\subsubsection{Behaviour of the Valve}

The development of electron microscopy allowed detailed observations of lymphatic valves in 1971 [45][46]. The absence of smooth muscle on the valve surface strengthened the observation by Florey that the valves appeared to move passively. In 1987, Mazzoni et al. published the first quantitive study of lymphatic valve structure [47]. Conducted 
on valves from the Spinotrapezium of rats, wax embedded cross-sections were prepared by microtome and imaged by light microscopy. Revealing the sparse collagen content of the leaflet and the "buttressing" of collagen around the annulus. Their paper considered the mechanics of the valve closure, hypothesising that the valves were driven by pressure differences between lymphangions. They also examined the opening and closing sequences and estimated the Reynolds number to be 0.0025 [47]. Mazzoni et al. did not consider wether there was an opening bias to the valves. Reddy's model considered these valves to be ideal and unbiased [32]. In 2008, MacDonald et al. included a valve bias term in their idealised valve model. In 2011, Davis et al. published a study on the valve gating a murine lymphatic vessels. This work expanded considerably on previous valve studies, observing behaviour in physiological media as well as considering the absence of tone by using calcium-free media [2]. They revealed that the valve gating depends on the trans-mural pressure as well as the presence of tone. Further discussion of previous modelling of valve behaviour can be found in Chapter 3 .

\subsection{Overview}

This work will seek to take techniques from engineering and computer science and use them to model aspects of the lymphatic system. This will be done with aim of improving the understanding of lymphatic disease and identifying new avenues of research. In Chapter 2 we will first consider a 3D approach to modelling lymphatic valve and wall. Lessons learned from this project will then inform Chapter 3. Where a 0D lumped model of a lymphangion is proposed. In Chapter 4, a chain of 0D lymphangions are used to form a vessel and these vessels connected to form a model of the superficial lymphatic system for the upper limb. Finally in Chapter 5 a consideration is then given to how lymphatic insufficiencies would effect limb shape and this is used to construct a shape based metric of lymphoedema which is then tested on limb scans from 21 unilateral lymphoedema patients. 



\section{Chapter 2}

\section{Modelling a Single Lymphangion: 3D FEA.}

Some work in this Chapter was published in The Journal of Biomechanics under the title "An Integrated geometric and mechanical analysis of an image-based lymphatic valve" in November of 2017 DOI:10.1016/j.jbiomech.2017.09.040. Permission to reproduce has been sought and given by Elsevier where appropriate.

\section{$2.1 \quad$ Introduction}

Lymphatic valves are essential to flow against gravity, their absence, or malformation in primary lymphoedema is associated with severe lymphatic insufficiency [48]. A previously published sensitivity analysis of lymphangion models has revealed that valve resistance is a determinant of lymphatic pumping function [42]. In reality, valve closing and opening is not instantaneous and this lymphatic resistance will evolve in time and in response to the trans-valvular pressure gradient. Understanding the dynamics of these lymphatic valves is complicated by their small size. Current published experimental 
data either occurs at a temporal resolution which allows the opening and closing to be observed [2] or at the spacial resolution which allows the geometry to be accurately captured [49]. The use of finite element modelling allows inference to be drawn between these scales by allowing statically images valves to be animated mathematically and the results compared to experimental data. Several authors have commented on the potential benefit of a lumped model of the lymphatic system [50]. A lumped model of a lymphangion is constructed by "lumping" behaviours together into a series of elements. These elements can be arranged in series or parallel to form networks. Constructing models this way overcomes the large computational expense involved in 3D CFD modelling. However, the model is dependent on the ability of these individual elements to recapitulate the behaviour of the $3 \mathrm{D}$ components they represent.

The differing approaches taken to "lumping" the valves into lumped model will be discussed in greater detail in Chapter 3. However, the greatest progress toward network scale modelling comes from the model constructed by Bertram et al. [40]. Briefly, Bertram et al. modelled the valves by imposing a fluid resistance, which was proportional to a logistic function of the applied trans-valvular pressure difference [40]. This approach has subsequently been used in network-scale models, by Jamalian et al. [43]. These valve models do not include experimentally demonstrated relationships between the trans-mural pressurisation and the trans-valvular pressure required to close the valves.

Previous work has considered the opening behaviour of three-dimensional idealised valves [51]. When the work in this chapter was undertaken, as far as the author was aware the only finite element analysis published about lymphatic valve closure, was flow around a two-dimensional valve [52]. Subsequently published work has considered $3 \mathrm{D}$ fluid structure interaction on idealised valves [53].

However, reconstruction from confocal imagery reveals several difficult to idealise features present on the valve, some of which have not been included in previous parametric models [49][51]. This process is intensive, and an idealised representative 
model would allow large parametric studies to find the relationships necessary to construct a lumped model of the lymphatic system. The valve represents the first 3D reconstruction of the lymphatic valve geometry. While, the features found may be artefacts of imaging, reconstruction and may not be found on technical or biological repeat of the experiment. They may also be unique to the mesentery or to mice in general. This work proceeds by including these features and leaving to future work the task of demonstrating their irrelevance. This work aimed to provide the first image-specific lymphatic valve and wall geometry and to use finite element analysis to study the closure of these compliant valves. Two metrics of valve closure were defined and sensitivity of this model is assessed against the shear modulus of the leaflet.

\subsection{Methods}

\subsubsection{Image Processing and Segmentation}

The image set used was acquired by Prof David Zawieja (Texas A\&M) and was produced by confocal imagery of a lymphangion isolated from the mesentery of a rat. A section of lymphatic vessel was extracted and placed in a calcium-free solution to prevent contraction. The vessel was cannulated and stained by intra-luminally loading with Cell Tracker Green CMFDA (5-Chloromethylfluorescein diacetate). The lymphangion was pressurised to a trans-mural pressure of $5 \mathrm{cmH}_{2} \mathrm{O}$, No trans-valvular pressure gradient was applied so that the valve was imaged in a rest state. The valve was scanned with a Leica AOBS SP2 confocal-multiphoton microscope with an U APO 40.0x1.15 W CORR objective, a $100 \mathrm{~mW} 488 \mathrm{~nm}$ laser was attenuated with an acousto-optical modulator, and acousto-optical beam-splitters were used to select the wavelengths from the emission spectrum between $510-525 \mu \mathrm{m}$. The vessel was aligned with the $x$ direction. A single $x-y$ confocal slice was acquired then the focal plane was advanced in the $z$-axis before acquiring the next $x-y$ image. The resolution in $x$ and $y$ is the same and dependent on the lens, scanning characteristics, confocal pinhole and staining intensity. However, the resolution in $z$ is lower and is dependent on the step size and confocal pinhole size. The 
scans represent a pack of 195 2D slices containing 512-by-512 pixels at a resolution of 0.6 -by- $0.6 \mu \mathrm{m}$. The distance between consecutive images is $1 \mu \mathrm{m}$. The image was converted to an intensity map which was then subjected to a median filter. A 307 micron length of wall containing a valve, was segmented from the image set by Dr Igor Sazonov (Swansea University). The valves length was 230 microns from the middle of the bases to the middle of the commissures. The diameter at the commissures is 160 microns, and the diameter at the base was 90 microns.

As can be seen in Figure 2.2.A, the images are very noisy, the objects blurred and the intensity is non-uniform. As the focal plan moves away from the laser source the fluorescent intensity decays exponentially due to scatter and adsorption. These issues complicate the reconstruction of the leaflets, which was ultimately performed manually, as described in Section 2.2.1.1. An overview of the lymphatic valve geometry is shown in Figure 2.1.A and B.

\subsubsection{Segmentation and Reconstruction}

The wall was segmented by an automatic active contour method implemented by Dr Igor Sazonov (Swansea University). In order to achieve this, we introduce the Cartesian coordinates: $x$ and $y$ in horizontal and vertical directions, respectively, in every slice and the $z$-axis, is the depth of a slice. Then wall contours are arranged in every $z$ - $y$ slice, see Figure 2.2.B. The initial contour in the first $z-y$ slice is an ellipse set manually by four points on the image in the middle of the wall. The contour displaces along its normal, moving up intensity gradients and stopping when it reaches the maximal intensity. In all subsequent slices, the final contour of the previous slice is taken as the initial. Thus the procedure is practically automatic except the setting of the first four points. When this procedure is completed the result is a series of points with each point representing a local maxima of intensity. Together these points describe a surface which is assumed to be at the centre of the wall. The thickness of the wall can then be determined by looking for the highest gradient of decay each side of the central surface. The wall surface is also smoothed axially along the surface to remove noise effects using a modified Laplacian 


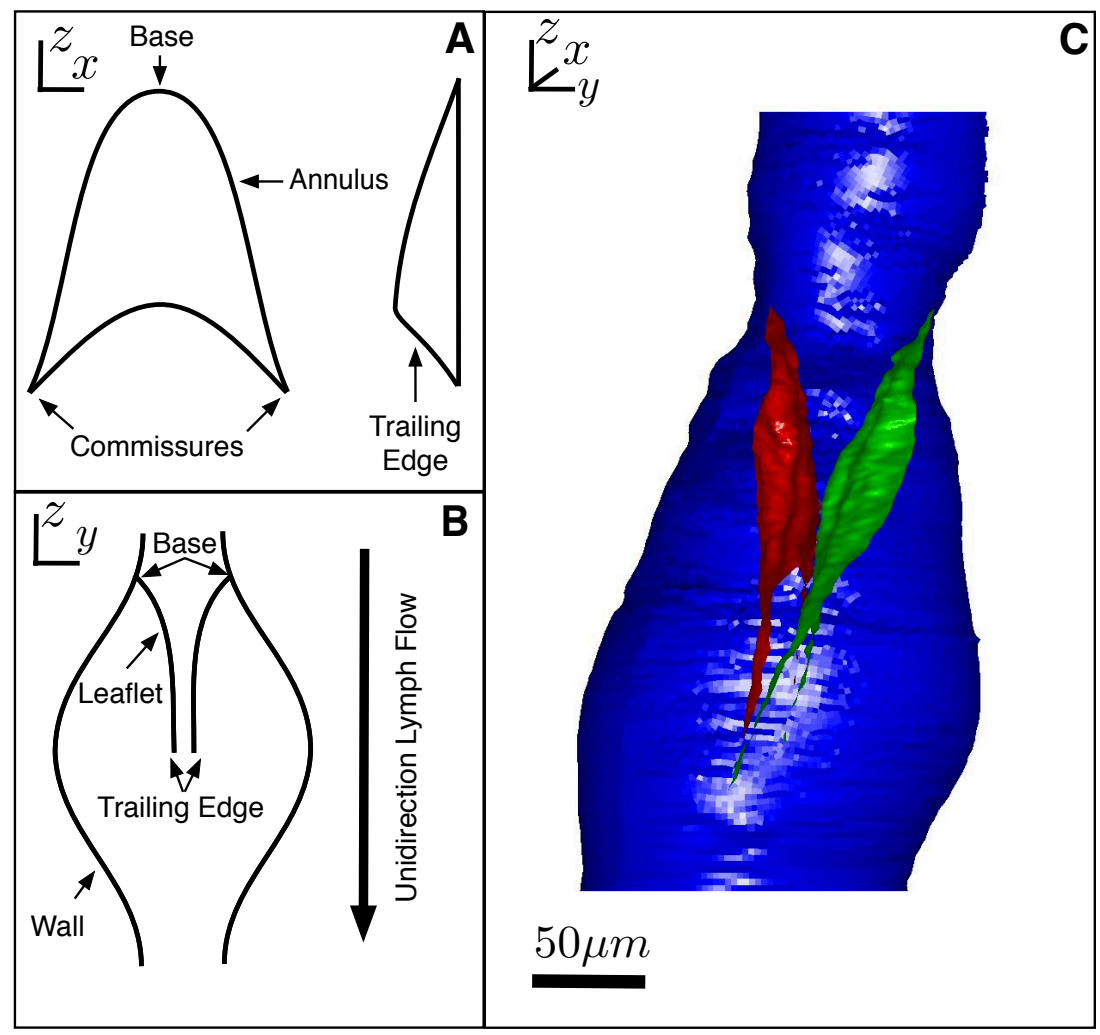

Figure 2.1: An overview of the lymphatic valve configuration and nomenclature. A) Nomenclature for the valve leaflets. B) The configuration of the leaflets to form the valve. C) Shows the Segmented structures of a subject-specific valve. Leaflets are shown in red and green, and the wall is shown in blue.

smooth algorithm. The Laplacian smooth algorithm usually displaces each point to the average of its neighbours. The method of segmentation results in each non-boundary point having two axial neighbours and two radial neighbours. The modified smooth algorithm moved each point to the average radial position of its axial neighbours. The smoothed wall geometry is shown in blue in Figure 2.1.C.

The leaflets were also segmented by Dr Igor Sazonov (Swansea University) using the following method. First, a separation line is manually placed between the two leaflets on each slice. These lines separate the voxels into two groups, those above the line belong to the top leaflet, those below the line belong to the bottom leaflet. The intensity for each leaflet is summed in the $z$ direction for both sides of the separation line creating two $2 \mathrm{D}$ images which reveal the outline of the leaflets. The trailing edges of the 


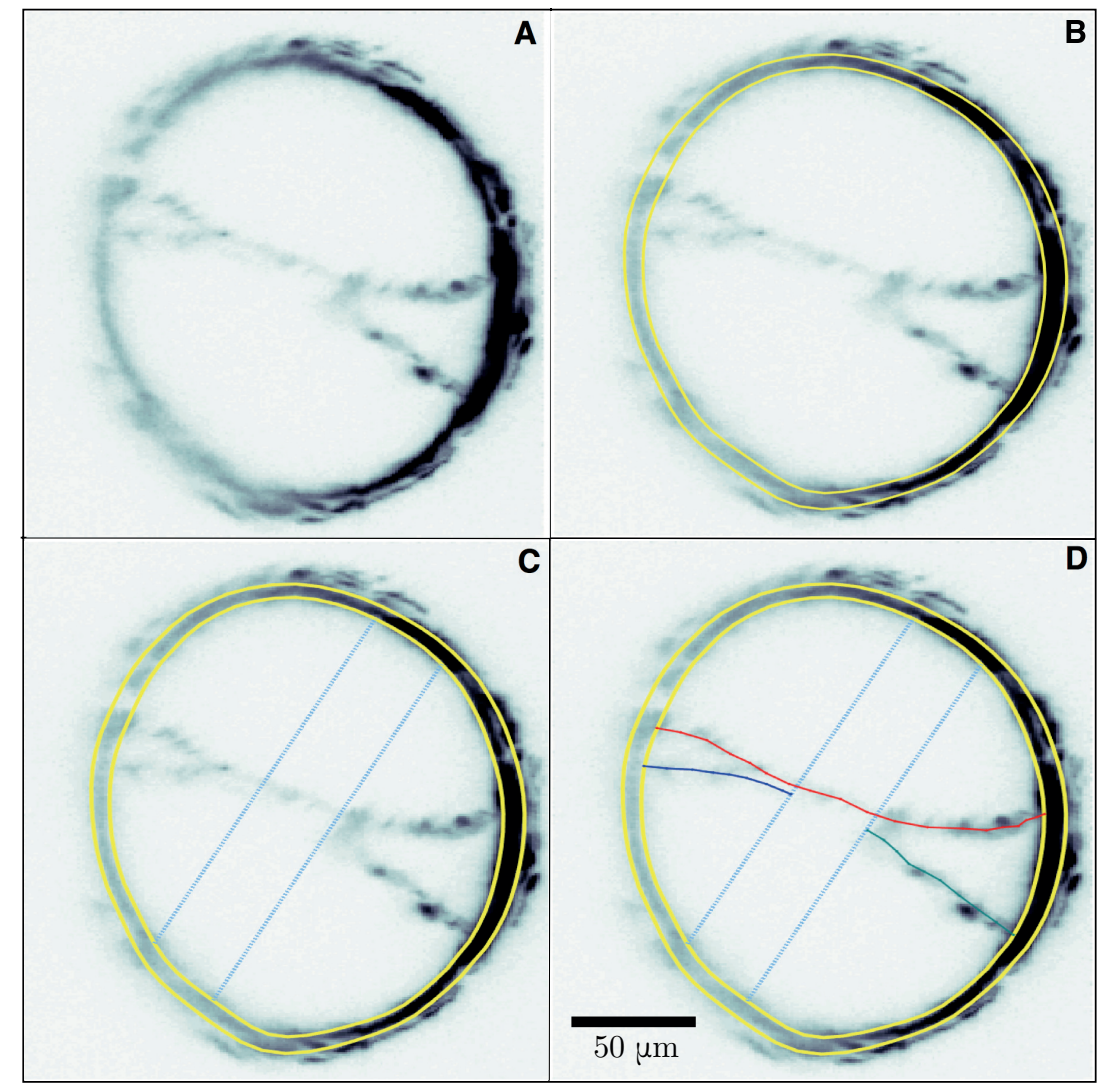

Figure 2.2: A series of z-y slices illustrating a step by step overview of the segmentation process. A) A z-y image slice. B) A segmentation of the wall (yellow). C) The guidelines of leaflet edges used in reconstruction (cyan). D) Manual reconstruction of the leaflet structures (red and blue).

valve leaflets are traced and used as a guide for manual reconstruction of the leaflets. A vector is constructed such that it is roughly perpendicular to the valve in the $z-y$ plane, the guidelines are parallel to this vector, as can be seen in Figure 2.2.C. For each slice, the user places a number of vertices resulting in a polygonal chain that describes the shape of the leaflet, see Figure 2.2.D. These points can then be post-processed and meshed to obtain the final geometry, see Figure 2.2.C.

\subsubsection{Material Properties of the Wall and Leaflets.}

The small size of lymphangions prohibits the use of classical methods to find material properties for the leaflet and the wall. The composition of lymphangions has also been 
established by immunostaining; with the leaflet consisting predominantly of elastin and the wall a collagen-elastin composite with collagen reinforcement at the annulus [1]. Existing experimentation on rats has found a highly non-linear pressure-diameter relationship for lymphangions, Rahbar et. al. fitted a pressure-diameter relationship of the form [1]:

$$
P_{\text {mural }}(D)=P_{0}\left(\exp \left[S_{p}\left(\frac{D}{D_{0}}-1\right)\right]-0.001\left(\frac{D}{D_{0}}\right)^{-3}+0.05\right)
$$

Where $P_{\text {mural }}$ represents the trans-mural pressure in $\mathrm{cmH}_{2} \mathrm{O}, D$ represents the vessel diameter in $\mu m, S_{p}$ the is a shape parameter which increases the rate of strain-stiffening and $D_{0}$ the normalising diameter measured at $P_{\text {mural }}=P_{0}$. For lymphangion sections taken upstream of valves the mean values for $P_{0}, S_{p}$ and $D_{0}$ were 18.0, 20.4 and 157.4 respectively. It is important to note that the normalising pressure, $P_{0}$, and diameter, $D_{0}$, are the largest considered. Ideally a microstructure motivated constitutive model of the lymphangion wall would be used, discussed in Section 1.3.1. However, experimentation is complicated by the collapse of the wall in a stress-free condition. When the lymphangion is un-stressed it collapses, preventing imaging and experimental analysis. As a result all imaging is conducted with a certain amount of pre-stress in the wall and leaflet. Material models have to account for this pre-stress. For linear material models the load conditions can be adjusted to remove the imaged load. For non-linear material models the stress-state of the lymphangion has to be known before analysis. An attempt to account for this without the complexity of an inverse-FEA study to find this stress is made below.

Rahbar et al. compared fits of their model for lymphangion sections upstream and downstream of a valve, the greatest change in parameters observed was $16 \%$ [1]. As the leaflet is predominantly devoid of collagen and much thinner than the wall. We take the assumption that the wall is dominant over the leaflets and the leaflets do not effect the motion of the wall. This allows a method by which representative wall motion can be achieved through an elasto-plastic model whereby the wall was considered very stiff elastically, and artificial plastic deformation was used to model the non-linear component 
of the behaviour described in Eq 2.1. This model allows representative wall motion for use in leaflet study. Firstly the wall is considered to be a homogenous thin walled cylinder described in $\{\Theta, R\}$ as $0<\Theta \leq 2 \pi, R=r\left(P_{\text {mural }}\right)$ with associated thickness, $t\left(P_{\text {mural }}\right)$; where $r$ is the radius of the wall at pressure $P_{\text {mural }}$. The assumption of symmetry allows only displacement $u$ in $R$. The assumption of incompressibility suggests that cross-sectional area is conserved.

$$
\pi\left[r\left(P_{\text {mural }}\right)+\frac{t\left(P_{\text {mural }}\right)}{2}\right]^{2}-\pi\left[r\left(P_{\text {mural }}\right)-\frac{t\left(P_{\text {mural }}\right)}{2}\right]^{2}=\text { Const }
$$

The principle strains can be written as:

$$
\boldsymbol{\epsilon}_{\Theta}=\frac{u}{r\left(P_{\text {mural }}\right)}, \boldsymbol{\epsilon}_{R}=\frac{t-t\left(P_{\text {mural }}\right)}{t\left(P_{\text {mural }}\right)}
$$

The principle stresses can be written as:

$$
\boldsymbol{\sigma}_{\Theta}=\frac{r\left(P_{\text {mural }}\right) P_{\text {mural }}}{t\left(P_{\text {mural }}\right)}, \boldsymbol{\sigma}_{R}=\frac{-P_{\text {mural }}}{2}
$$

This allows the creation of a von-Mises equivalent stress-strain relationship. The prestress of the cylinder was modelled by the addition of the existing stress; where $P_{1}$ is the trans-mural pressure at imaging.

$$
\boldsymbol{\sigma}_{v M}\left(P_{\text {mural }}\right)=\boldsymbol{\sigma}_{v M}\left(P_{1}\right)+\boldsymbol{\sigma}_{v M}\left(P_{\text {mural }}-P_{1}\right)
$$

This relationship was decomposed into a linear-elastic component representing the gradient at $P_{\text {mural }}=P_{0}$, in this case, a modulus of $413 \mathrm{kPa}$ and the remaining strain through artificial plastic deformation. This model was implemented in ANSYS workbench, and the wall was pressurised to $18 \mathrm{cmH}_{2} \mathrm{O}$ to cover the full range considered by Rahbar et al. [1]. Shown below in Figure 2.3 is the fit described in Eq 2.1 compared to the mean $D / D_{0}$ for 10 equally sized bands through the wall geometry. As can be seen, the reconstructed wall is slightly more compliant than the fit. In the absence of collagen an incompressible neo-Hookean model was used for the leaflets with a shear modulus of $50 \mathrm{kPa}$. This value has been used in other studies of lymphangions but is 


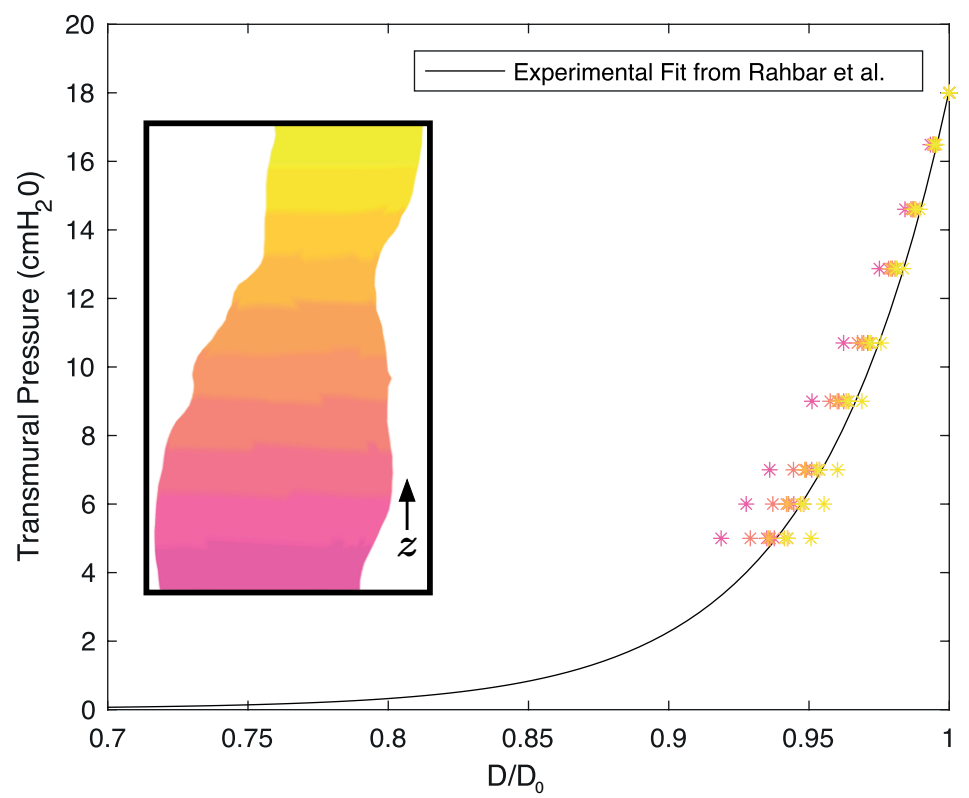

Figure 2.3: A plot of the mean $D / D_{0}$ for ten equally spaced bands taken axially along the wall geometry during pressurisation. Colour indicates the location of the bands, shown on the lymphangion geometry in the sub-figure. The black line represents the pressure diameter relationship found by Rahbar et al. [1].

based on experimental data from arterial elastin [51]. The sensitivity of this value was assessed in the material properties study.

\subsubsection{FEA Model Setup}

A steady-state elastic finite element analysis was used to assess the pressure required to close the valve. As immediately after valve closure there is no flow through the lymphangion this valve can be found without the need for a fluid-structure interaction study. ANSYS Workbench was used to solve the FEA problems. Meshes of varying densities were originally created for the reconstructed geometry. These meshes were then converged to a mean 1 percent relative error by the Euclidean norm of the entire displacement solution field, at a meshing density of 1 node per $3 \mu m^{2}$. The results of the manifold harmonic analysis were re-meshed, to preserve mesh quality, at a finer level of 1 node per $\mu m^{2}$. For the flexible wall studies an initial mesh of 1 node per $5 \mu m^{2}$ was 
refined until the pressure required to close the valve and the retrograde displacement of volume had a relative error of less than one percent. For every mechanical model, the leaflet was represented as a series of linear quadrilateral shells each with an associated thickness of 5 microns. Contact between the leaflets was modelled by an augmented Lagrange algorithm; the normal stiffness was relaxed to 0.5 of the default setting to allow convergence and an under-relaxation between equilibrium iterations of -0.5 the time derivative of displacement was used. The leaflets were considered rough, and slipping was discouraged through a penalty scheme. The effects of large displacement were modelled through the ramped application of load in up to 50 steps, during the static analysis. Both leaflets were pressurised with a trans-valvular pressure difference of $5 \mathrm{cmH}_{2} \mathrm{O}$ in order to close the valve. The afferent and efferent ends of the wall were held in-plane, and one node on the afferent end of the wall was fixed in space. The leaflets were bound to the wall by coupling displacements to nearest nodes on the wall. Rotations were also coupled in order to represent collagen buttressing of the leaflet previously observed [1].

\subsection{Results}

Three phases of displacement can be identified during the application of an increasing trans-valvular pressure gradient. The mean of the peak axial displacement of the trailing edge for both leaflets is shown in Figure 2.4. Firstly, the unimpeded motion of the trailing edges toward each other; secondly, a transition as the trailing edges start to coapt, and finally the leaflets deform against one another and the valve seal develops. As shown in Figure 2.4.D these regions can be delineated by the first trans-valvular pressure at which initial contact between the two leaflets occurs and closure of the valve, defined as the first load step for which a contiguous area spanned a leaflet which was within half a micron of the other. To examine the effect of the wall motion the trans-valvular pressure required to close was compared with imposed wall motion and with the annulus fixed in place. The trans-valvular pressure required to close the valve rose from 1.56 to 2.52 $\mathrm{cmH}_{2} \mathrm{O}$. A study was performed to assess the sensitivity of the model to changes in the leaflet shear modulus. The wall was included in this study though its material properties 


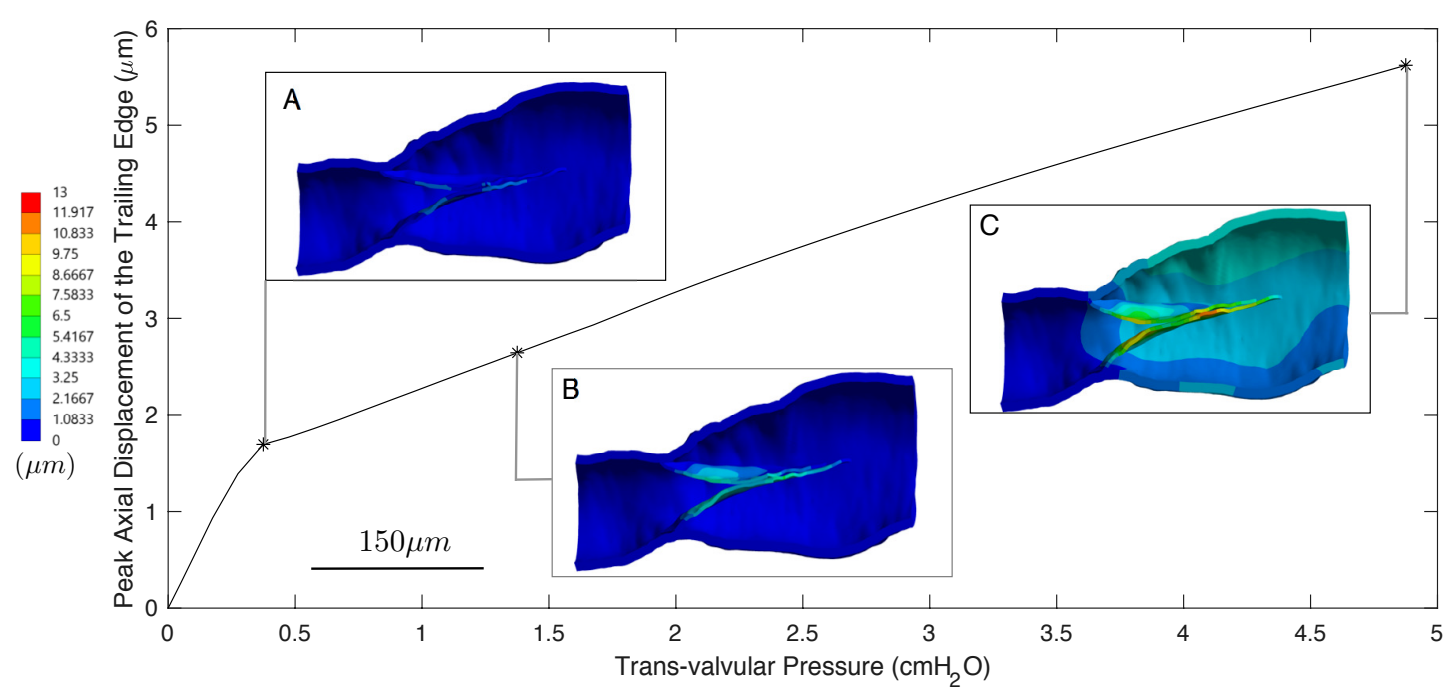

FiguRE 2.4: A plot of the peak axial displacement of the trailing edge against transvalvular pressure. Also shown is the original valve at three different trans-valvular pressures with the Euclidean displacement field coloured. A marks the first contact between the leaflets. $\mathrm{B}$ marks the point of minimal orifice area and $\mathrm{C}$ is the maximum applied trans-valvular pressure.

were kept constant as they were chosen to give representative motion, as discussed in Section 2.2.2. The shear modulus for the leaflets was varied from $10 \mathrm{kPa}$ to $60 \mathrm{kPa}$. A trans-valvular pressure of $5 \mathrm{cmH}_{2} \mathrm{O}$ was applied to the valve in a closing manner. Shown below in Figure 2.5 are the effects of leaflet shear modulus on the displacement of the leaflet. There is a clear relationship between decreasing stiffness and a reduced pressure required to close the valve. Davis et al. used calcium-free media to study lymphatic valve gating without tone comparing those results to the experimental data for a comparable valve it would appear the shear modulus is much less the $50 \mathrm{kPa}[2]$. After closure of the valve, the leaflets continue to displace axially under the applied trans-valvular pressure which results in retrograde displacement of the fluid within the lymphangion. This quantity is expected to play no role in lymphatic pumping however it is a metric of valve seal that is suited to subject-specific modelling as it doesn't rely on finding the apex of the commissure or defining a plane perpendicular to the commissures. Geometry specific quantities are easy to define for idealised geometries but difficult for subject specific geometries given their asymmetry and idiosyncrasies. This volume was estimated by calculating the volume between the two leaflets in the imaged state and 
then after the application of a $5 \mathrm{cmH}_{2} \mathrm{O}$ trans-valvular pressure difference in a closing manner. The change in retrograde volume displaced at $5 \mathrm{cmH}_{2} \mathrm{O}$ is shown in Figure 2.6. As can be expected the retrograde volume displaced is a function of the trans-valvular pressure required to close, but it also takes into account the compliance of the geometry.

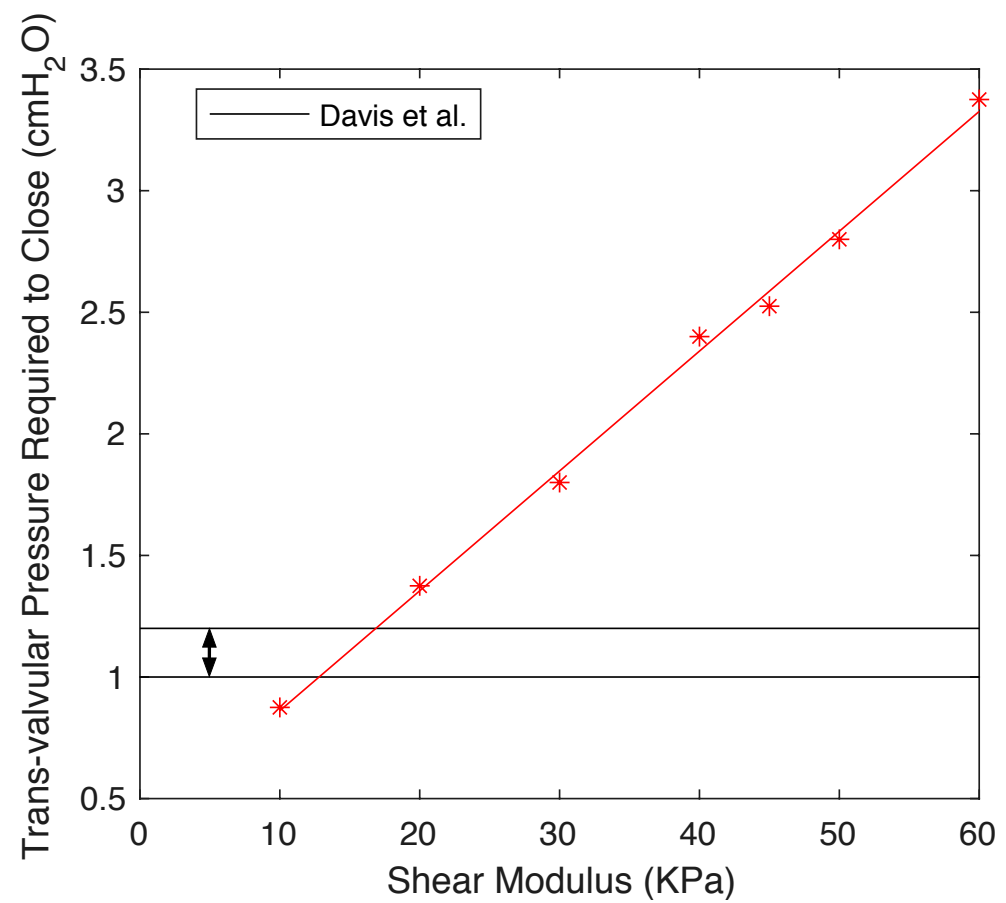

FiguRE 2.5: Trans-valvular pressure required to close the valve for differing leaflet shear moduli. The black lines represent experimental data for a lymphatic valve without tone and a trans-mural pressure of $5 \mathrm{cmH}_{2} \mathrm{O}[2]$ 


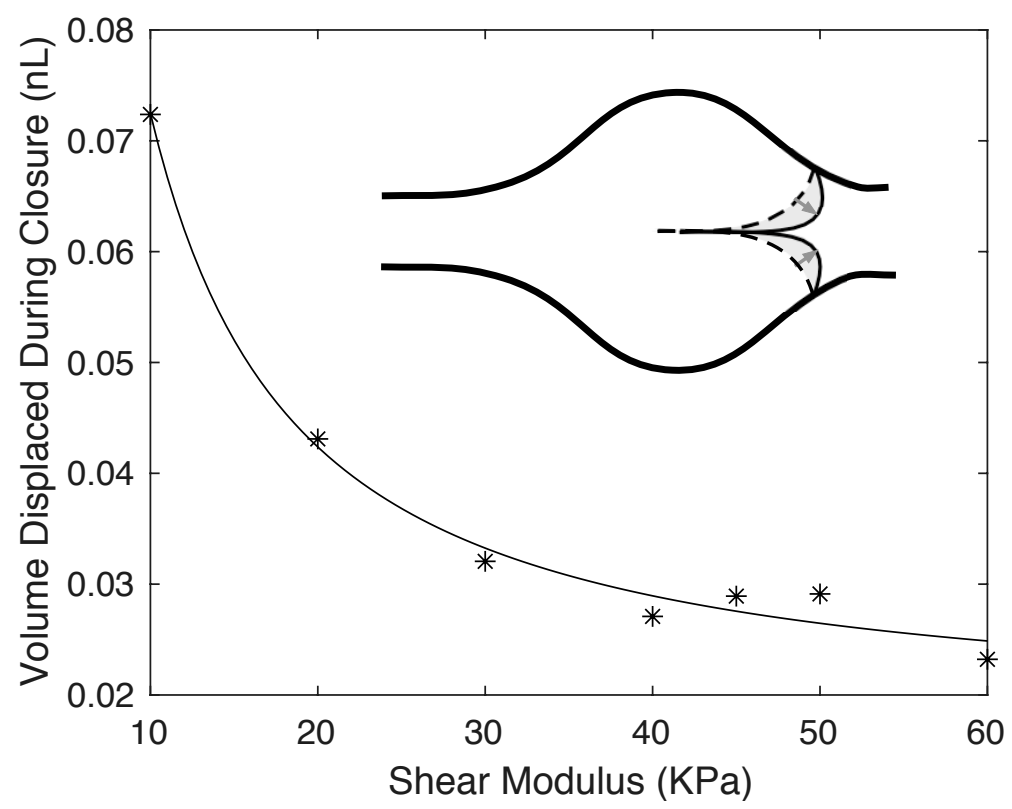

FIGURE 2.6: Retrograde volume displaced for differing leaflet shear moduli.

\subsection{Discussion}

A subject-specific model of a lymphangion has been presented and metrics of valve closure which account for the difficulties associated with subject-specific modelling proposed. A method of achieving representative wall motion has been proposed and the resultant $3.55 \%$ increase in diameter, as estimated by the pressure-diameter relationship, causes a two-fold increase in the trans-valvular pressure required to close the valve. Indicating that wall motion plays a significant role in valve gating. The estimated shear modulus for the leaflet of $50 \mathrm{kPa}$ based on arterial studies of elastin and previously used in studies of lymphatic valves appears to be an over-estimation [51]. There are several limitations to this study. The contractile nature of the wall was not included in any of the mechanical models. Secondly, the material properties of the wall in this study were designed only to produce representative motion, the creation of a constitutive model based on the interaction between collagen and elastin at various strain-states within the wall would allow for a greater consideration of its behaviour. The properties describing 
the elastin network in lymphatic leaflets are still unknown and might affect valve behaviour. Experimental determination of these properties is very challenging given the small size of the leaflets. Hence, future work could address these limitations by performing a material characterisation of the valves imaged in two positions and a two-way fluid-structure interaction study. 


\section{Chapter 3}

\section{Developing a 0D Lumped Model for the Lymphatic System: From Lymphangion to Limb.}

Mathematical modelling of the lymphatic system was first undertaken by Reddy et al. published in 1976 [32]. The model of Reddy et al. considered the entire human lymphatic system as 28 lumped lymphatic vessels. Since that work, studies have continued to develop the lumped approach down to the single lymphangion scale, while others have developed continuum mechanic models [50]. However, the largest models have considered networks of less than 100 lymphangions [43]. It is hoped that larger scale lymphatic models will offer a holistic understanding of disease, which will allow for greater understanding of experimental results and can elucidate the relationship between pathology and insufficiency which underpins lymphoedema. In this chapter an attempt will be made to expand lymphangion-scale lymphatic modelling to the tens of thousands of lymphangions necessary to model limb or organ scale lymphatic networks. This chapter presents the methods used to form a model with occasional results to illustrate behaviours. As a result the chapter is not presented in the methods, results, discussion structure used throughout the rest of the work; instead methods are 
grouped thematically. Throughout this chapter previous methods will be debated, new approaches considered, and a model for lymphatic vessels at the limb scale proposed. Chapter 4 will explore the behaviour of this model, present results, and discuss their implications.

Lumped lymphatic models "lump" basic elements together to produce a system of equations which can be solved to find the flows, diameters and pressures within the lymphatic system. The term "0D lumped model" describes models which have no remaining spatial dimensions at assembly. For example, compare the 1D approach published by MacDonald et al. to the 0D approach published by Bertram et al. [34][55]. Both reduce the Navier-Stokes equations to a 1D tube; MacDonald integrates this equation across a series of computational cells, allowing the parameters to change along the length; Bertram et al, represent them as Hägen-Poiseuille style resistance that has already been integrated along the length of the vessel. The first approach yields a 1D lumped model where additional elements will capture additional detail, provided the parameters, such as contraction state, change along the length. The second approach yields a 0D lumped model where the parameters are fixed along the element. Given the aim of this study is to produce the first limb-scale model of the lymphatic system the 0D approach is taken, as it offers reduced computational expense. As has been previously noted contractions appear to be almost instantaneous between lymphangions at the lymphangion scale [50]. Apart from the valve regions, lymphangions are assumed to be constant diameter. Meaning there may not be the clear benefit to a $1 \mathrm{D}$ approach that has been realised in cardiovascular modelling [56].

Beyond modelling the flow through the pipe like geometries of the lymphatic system, there are three essential properties of lymphangions which we seek to replicate. 1) Lymphangions are compliant. 2) Lymphangions are contractile. 3) Lymphangions are valved. In this section we will explore each of these properties in turn, discuss previous attempts to recapitulate observations of these properties, and propose modifications and adjustments which may better suit our aims of limb-scale lymphatic modelling. Each individual aspect of the model is explained in turn before an explanation of the assembly. 
A circuit equivalent overview of a lymphangion is shown below, Figure 3.1. It consists of four fundamental building blocks. The flow through the lymphangion is restricted by two resistors, a capacitance at the central pressure node is used to represent a compliance, and a variable resistor is used to represent a valve.

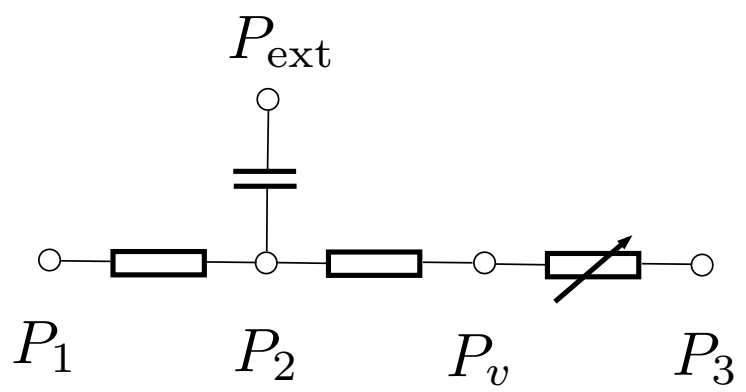

FiguRE 3.1: A circuit equivalent overview of a lymphangion

\subsection{Modelling Flow}

All lumped lymphatic models, of which the author is aware, treat lymph as an incompressible fluid with an axisymmetric flow. With the notable exception of Quick et al. an assumption is also made for quasi-steady flow [33]. These assumptions allow the Hägen-Poiseuille simplification of the Navier-Stokes equations. In order to examine these assumptions let us divide the flow through a lymphangion into three regions; Flow distant from a valve, flow around a valve and flow through a valve. In the first case the very low Reynolds numbers support a quasi-steady approach where changes in velocity do not have significant inertial consequences - in 1987 Mazzoni et al. estimated the Reynolds number for a lymphangion in the spinotrapezium muscle of a rat to be 0.0025. They assumed the length scale was the width of the vessel, the velocity scale was the mean fluid velocity, and the viscosity was twice that of water [47]. An attempt to find a scaling for Reynolds numbers within the lymphatic system is made below. For a thin-walled cylinder the relationship between wall tension and internal pressure is as follows.

$$
T=\frac{P D}{2}
$$


With $T$ being tension in the wall, $P$ being the internal pressure, and $D$ representing the diameter. If we assume that tension is directly proportional to the wall thickness and thickness directly proportional to diameter then the internal pressure for lymphangions should be scale-free. This concept has support in literature; Davis et al's valve experiments in the mesentery of mice and McHale et al's valve experiments in the mesentery of cows have approximately the same pressure scale despite the orders of magnitude difference in the diameter of the lymphangions. If we assume that this pressure alone drives flow, then we can say that lymphangion fluid velocity is proportional to the square of diameter. Taking a characteristic length as diameter, we can say that the Reynolds number scales with the third power of diameter.

$$
\operatorname{Re} \propto D^{3}
$$

This would imply that for lymphangions with diameter less than one millimetre the Reynolds number is expected to be less than 100. This assumption covers all lymphangion diameters considered in this work.

The above calculation is based on mean velocities and diameters which change over contraction cycles - of the order of 1-10 seconds [55]. The high length-diameter ratio and cylindrical nature of this region also support an assumption of axisymmetry. In the second region the assumption of quasi-steady flow may also be challenged. The valve motions and velocities associated with the ejection phase of lymphangion pumping occur over much shorter periods than the contraction cycles - of the order of 0.1-1 seconds [2]. Whilst Quick et al. expected viscosity to dominate in their consideration of lumped lymphatic modelling, they preserved an inertial term in order to correctly capture ejections [33]. Bertram et al. however, later omitted this term arguing that the Reynolds numbers where sufficiently small to justify doing so [55]. This assumption has continued in later works [43]. Prior to this consideration by Bertram et al., Rahbar through a 3D CFD model of a radial contracting lymphangion found the wall shear stresses to agree with those predicted by a Poiseuille model to within 4\% [39]. The pressure-flow relationship stated by Quick et al. is shown below, with the inertial term 
omitted by Bertram identified [33][40].

$$
\Delta P=\underbrace{\frac{8 \eta L}{\pi r^{4}} Q}_{\text {Viscous Term }}+\underbrace{\frac{\rho L}{\pi r^{2}} \frac{d Q}{d t}}_{\text {Inertial Term }}
$$

In flow through the valve itself the assumption of axisymmetry may be better restated as the assumption that the resistance of the valved section has an equivalent Hägen-Poiseuille resistance. The nature of this relationship may not be trivial, Wilson et al. performed 3D CFD simulations of idealised lymphangions [51]. As could be expected increasing the sinus-to-root ratio increases the area available for flow and reduces the resistance. Wilson et al. found, within a physiological range of sinus to root ratios for rat mesenteric lymphangions, this effect was so prominent that the resistance of the valve-sinus assembly was lower than the Hägen-Poiseuille resistance of a tube of root diameter. So in these cases, because of the sinus, the inclusion of the valve lowers the overall resistance of the lymphangion.

It was common in earlier considerations of lymphangions to omit the resistance in the valve section and assume an ideal valve model where flow is permitted, unimpeded, when pressure gradients support it and completely prohibited otherwise [57][34].

\subsection{Lymphangion Valves}

Modern attempts at lumped modelling of the lymphatic system have sought to move away from idealised valves for three reasons. Firstly, to capture the observed open-biased nature of the valve. Secondly, to avoid temporal discontinuities in valve state. Finally, constraining the flow to zero leads to a infinite resistance which can cause numeric issues. This second factor is significant in correctly capturing the inertial effects of ejection. Bertram et al. used valves whose resistance was directly proportional to a logistic function of the trans-valvular pressure gradient with a bias. This allowed a finite resistance, which is a function of the trans-valvular pressure gradient. Whilst this produces temporally-smooth resistances, they apply instantaneously [55]. Contarino 
and Toro modelled the valve through an ODE where the change in valve state was proportional to the trans-valvular pressure gradient [35]. This represents the input-lag property of the leaflets which will be transiently deformed to closure by the flow field that occurs as a result of the trans-valvular pressure gradient. They also allowed for different behaviours in the opening and closing modes representing the hysteresis found by Davis et al. [2]. However instead of modulating a resistance, Contarino and Toro modulate the cross-sectional area from which the resistance is calculated [35]. The result is that the valve state is proportional to the square of the conductance of the section. This property is more closely inspired by the mechanical nature of the valves but is difficult to compare to other models and poses numerical issues due to the unbounded resistances it can generate if that area is allowed to be zero.

A new model is proposed; which, like Bertram et al., modulates a resistance directly but takes the form of that used by Contarino and Toro [55][35]. An additional modification is taken in order to replicate the behaviour observed by Davis et al. that the valve gating is a function of the trans-mural pressure. As the annulus of the valve is stretched the open-bias increases, this phenomenon was described in in Chapter 1 and explored in Chapter 2. The valve model, with the proposed modification, is shown below. With $\zeta$ representing the normalised valve resistance, $P_{\mathrm{tv}}$ the trans-valvular pressure, $P_{\text {bias }}\left(P_{\mathrm{tm}}\right)$ the trans-valvular pressure required to close the valve.

$$
\frac{d \zeta}{d t}= \begin{cases}k_{v o}(1-\zeta)\left(P_{\mathrm{tv}}+P_{\mathrm{bias}}\left(P_{\mathrm{tm}}\right)\right) & P_{\mathrm{tv}}+P_{\mathrm{bias}}\left(P_{\mathrm{tm}}\right) \geq 0, \text { Opening } \\ k_{v c} \zeta\left(P_{\mathrm{tv}}+P_{\mathrm{bias}}\left(P_{\mathrm{tm}}\right)\right) & \text { else, Closing }\end{cases}
$$

To show the differences between the two models a simple demonstration is presented in Figure 3.2. An expression for $P_{\text {bias }}$ is found by fitting against the results found by Davis et al. [2]. The data presented in Davis et al. is recapitulated in Figure 3.2.A. In the original work a power-law fit was used. While this would appear to be correct, a reasonable approximation is achieved with a linear fit as shown in Figure 3.2.A. To represent a contracting lymphangion the trans-valvular pressure, $P_{\mathrm{tv}}$, is represented 
by a sine wave with a period of 25 seconds and a amplitude of $2 \mathrm{cmH}_{2} \mathrm{O}$. The transmural pressure linearly increases from 0 to $20 \mathrm{cmH}_{2} \mathrm{O}$ over four contraction cycles. The imposed trans-mural pressure and trans-valvular pressures can be seen in Figure 3.2.C, with the resultant valve closing and opening shown for the Bertram model and the proposed model. As can be seen, in the proposed model, there is a valve bias which changes with trans-mural pressure.

The normalised resistance, $\zeta$, is calculated for these conditions using the eq 3.4 , a hysteresis is included by setting $k_{v o}=0.5 \mathrm{cmH}_{2} \mathrm{O}^{-1} s^{-1}$ and $k_{v c}=1 \mathrm{cmH}_{2} \mathrm{O}^{-1} s^{-1}$ . In Figure 3.2.B, this resistance can be seen for the four cycles. The proposed model is coloured for time and the model used by Bertram is shown in black. The hysteresis of valve motion is reduced due to the higher trans-valvular pressures during opening and closing.

It can be expected that there would be much site to site and species to species variation in the relationship between trans-mural pressure and valve biasing. Such a study has not been performed outside of the lymphangions of mice. It is also important to note that allowing the valve state to evolve in time complicates the measurement of the pressure required to close the valve. Davis et al. measured valve closure by thresholding for the total intensity in images taken between the valves. When this threshold is met the valves have closed, but the pressure which closed them occurred several hundredths of a second before. In a spontaneously contracting lymphangion the difference in pressure between the start and end of a valve motion is likely to be significant. 


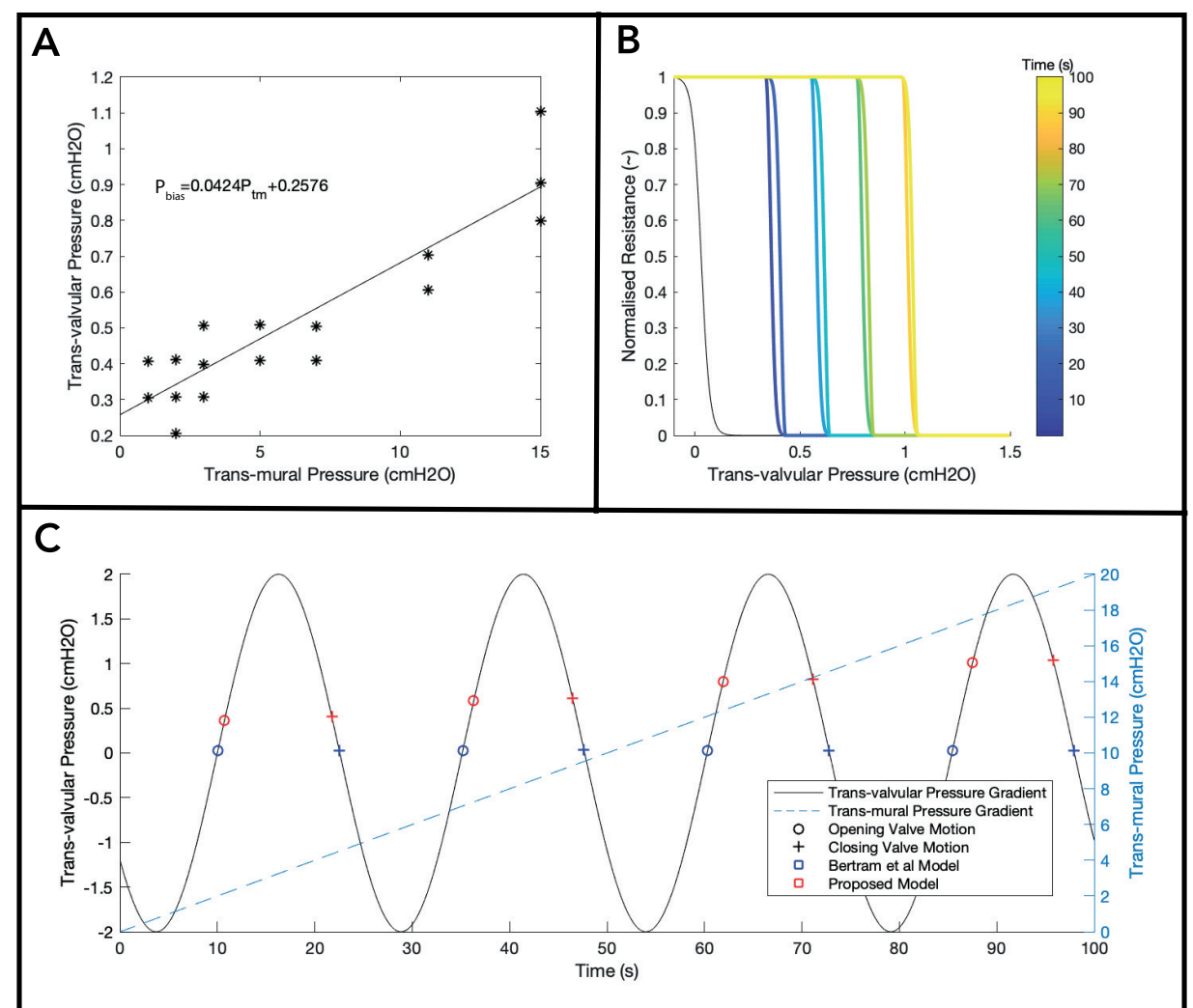

Figure 3.2: A) The trans-valvular pressure required to close the valves at differing trans-mural pressures, reproduced from Davis et al. [2]. The black line indicates a linear fit with the equation shown. B) Shows a trans-valvular pressure - normalised resistance plot for the valve demonstration shown in C. The line colour shows the time allowing the increase in valve open bias present at higher pressures. The hysteresis can be seen which appears diminished at higher pressures. Shown, in black is the relationship in the Bertram et al. model without hysteresis or trans-mural pressure dependent biasing. C) Shows a sinusoidal trans-valvular pressure with an increasing trans-mural pressure. Markers indicate a change in valve states for the Bertram et al. and proposed model, found by identifying when the normalised resistance crosses a $50 \%$ threshold

\subsection{Imposing Compliance}

Material models for lymphangions in 0D lumped modelling vary in their rigour and behaviour, as was discussed in Chapter 1. For the same reasons laid out there we proceed using empirical pressure-diameter relationships. Bertram et al. created a similar model 
to that of Rahbar and Moore to fit experimental pressure-diameter relationships, shown below with change in nomenclature.

$P=P_{d}\left[c_{1}\left(\frac{D}{D_{d}}-c_{2}\right)^{2}+c_{3} \exp \left(c_{4}\left(\frac{D}{D_{d}}-c_{5}\right)\right)+c_{6}+c_{7}\left(\frac{D}{D_{d}}-c_{8}\right)+c_{9}\left(\frac{D}{D_{d}}\right)^{-3}\right]$

Where, $D$, represents the diameter, $D_{d}$, represents a scaling for diameter, $P$ represents pressure, $P_{d}$ represents a scaling for pressure and $c_{1-10}$ represent dimensionless fitting parameters. The pressure and diameter scalings are taken very close to the stress-free condition, 735 dynes $\mathrm{cm}^{-2}$ was the value fitted. Examining this relationship it can be decomposed into three phenomena. The first, $P_{\text {Strain-Stiffening is a highly non-linear }}^{*}$ stiffening associated with an elastin-collagen duty transition at higher stretches; the second, $P_{\text {Strain-Thinning }}^{*}$ represents the strain-thinning incipient to collapse; and the third, $P_{\text {Residual }}^{*}$ is the residual behaviour when those two are removed and represents a pressurediameter curve for a nearly linear thin-walled cylinder. The three decompositions are stated below and shown in Figure 3.3. It is important to note at collapse, $D=0$, this solution ceases to exist. Bertram added the strain-thinning term to better agree with experimental data collected by Davis et al which showed a decrease in vessel compliance as the vessel approached collapse. This term has an additional consequence of making complete collapse of the vessel impossible. This has several benefits from a numerical standpoint, however, it is unphysiological.

$$
\begin{gathered}
P_{\text {Strain-Thickening }}^{*}=c_{3} \exp \left(c_{4}\left(\frac{D}{c_{9}}-c_{5}\right)\right) \\
P_{\text {Strain-Stiffening }}^{*}=c_{10}\left(\frac{c_{9}}{D}\right)^{3} \\
P_{\text {Residual }}^{*}=\left(\frac{D}{c_{9}}-c_{2}\right)^{2}+c_{6}+c_{7}\left(\frac{D}{c_{9}}-c_{8}\right)
\end{gathered}
$$




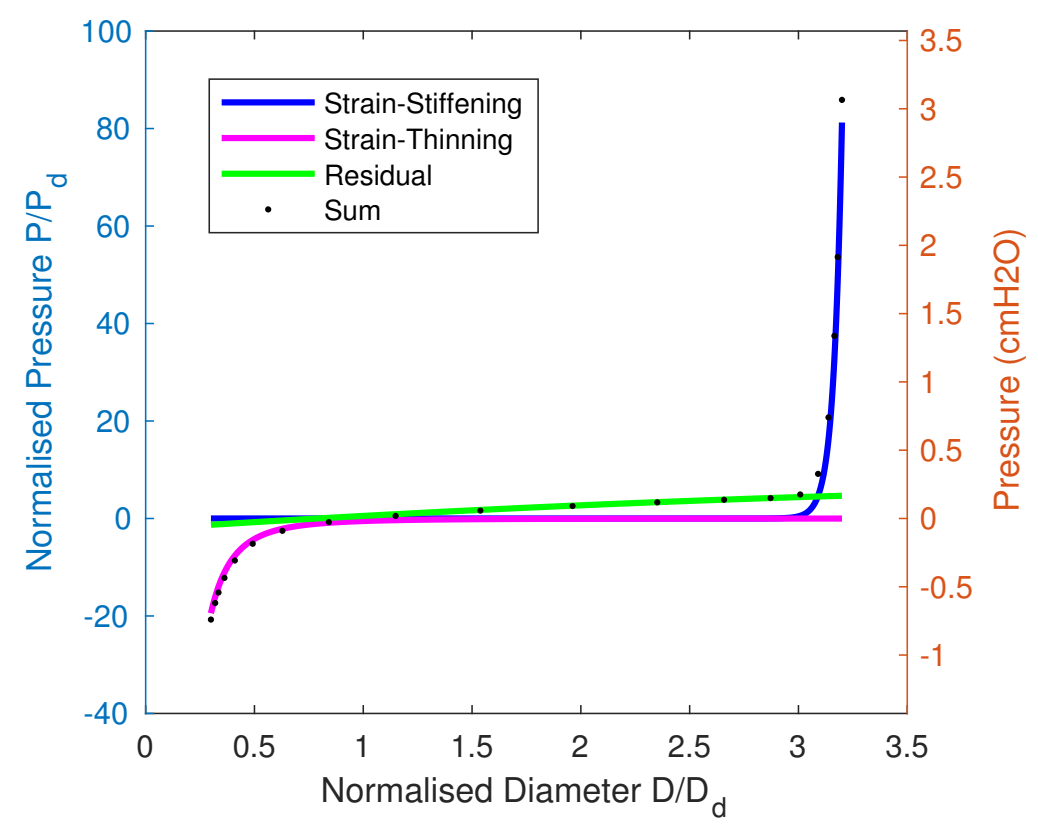

FiguRE 3.3: The passive pressure diameter relationship proposed by Rahbar decomposed into the following functions [1]. A strain-stiffening function at high stretches,

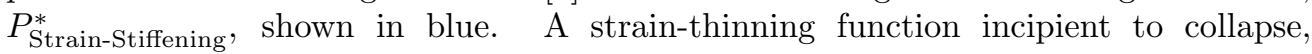
$P_{\text {Strain-Thinning, }}^{*}$, shown in magenta. $P_{\text {Residual }}^{*}$ the residual with the above functions removed, shown in green. Finally, the sum of the above three functions is shown in black. Left axis is the normalised pressure ratio. Right axis is the pressure if $P_{d}$ is 735 dynes $\mathrm{cm}^{-2}$

There are numerical issues with $P_{\text {Residual }}^{*}$. The quadratic form yields non unique solutions. This is resolved in a simplification proposed by Bertram which is stated below [40].

$$
P_{c}=P_{d} \frac{4}{15}\left[12 \exp \left(\frac{D}{D_{d}}-1\right)-11-\left(\frac{D}{D_{d}}\right)^{-3}\right]
$$

This can arrived at by setting $c_{1}=c_{2}=c_{7}=c_{8}=0, c_{4}=c_{5}=1, c_{6}=\frac{44}{15}, c_{3}=\frac{16}{5}$ and $c_{9}=\frac{4}{15}$. The condition of the resulting system can be further improved by constraining the diameter, $D$, to the range $D \rightarrow[\epsilon$,inf $]$, where $\epsilon$ is some small number. 


\subsection{Imposing Contraction}

Myocytes within lymphangion contract periodically in order to pump against pressure gradients and there are multiple ways of considering this mathematically. However, they each represent, implicitly or explicitly, an additional pressure which is a function of time and the lymphangion stress state, $f_{a}(\sigma, t)$.

$$
P_{c}(D)+f_{a}(\sigma(D), t)+P_{e x t}=P_{2}
$$

In one the first considerations of lymphangion-scale 1D modelling, McDonald et al. inspired by treatments of the vascular system proposed that this $f_{a}(D, t)$ be represented as a stress occurring due to a modulating stiffness [34]. By mapping this stiffness to a contraction state $0 \leq \chi \leq 1$ a dimensionless variable representing the contraction state could be imposed a priori or substituted for a more complex function of the lymphangion state which can include calcium and nitric oxide dynamics as recently used by Contarino and Toro [35].

\subsubsection{Modulating Tension}

An alternate method originally used in lymphatic modelling by Bertram et al. represents $f_{a}$ as a tension, which varies in time. It is important to note that if $f_{a}$ is just a function of $t$ it is insufficient to capture the behaviour of lymphangions [55]. Consider a circumferential tension, $M_{0}$, on a thin-walled cylinder of thickness, $\tau$. then the hoop stress $\sigma_{\theta}=\frac{M_{0}}{\tau}$ can be represented as a pressure $\frac{2 M_{0}}{D}$ This pressure can be made transient by some periodic function, with period $T, M_{t}(t+T)=M_{t}(t): \boldsymbol{R} \rightarrow[0,1]$

$$
f_{a}(D, t)=\frac{2 M_{0} M_{t}}{D}
$$


It is important to note that after the downstream valve has opened, if the lymphangion enters a collapse regime, $f_{P}(D)<0$ the contraction is unbounded.

$$
\lim _{D \rightarrow 0} f_{a}(D, t)=\infty
$$

Once $P_{\text {open }}$ is overcome the valve will collapse. It is also important to note that the diameter-time inflection does not agree with experimental observation during the start of contraction. Tension increases with the inverse of $D$, and increasing tension increases further decreases $D$. In order to prevent the collapsing behaviour in another work Bertram et al. made this tension a function of diameter thus $M_{0}$ is replaced with $M(D)$ which relates the tension to the strain state and prevents tension in the stress-free state [40]. This makes the hoop-stress a function of the strain-state which is functionally equivalent to the modulating stiffness approach. This method has been incorporated into network-scale models [43].

\subsubsection{Refined Modulating Stiffness Model}

The model proposed in this work builds on the above methods by considering an additional pressure term which represents the stress induced by the stretch of a coupled thin wall cylinder with modulating stiffness. This can be represented explicitly as an additional pressure term due to a coupled thin-walled cylinder. While, this cylinder will be given linear properties they will be derived by an examination of experimental results. Consider the hoop stress in a thin cylinder of diameter $D$ experiencing a hoop stretch $\lambda_{\theta}^{\mathrm{Myo}}$.

$$
\sigma_{\theta}=E_{\max } M_{t}\left(\lambda_{\theta}^{\mathrm{Myo}}-1\right)
$$

Assuming that myocytes do not function in compression, it is assumed there is some diameter represented as $\alpha_{\mathrm{sf}} D_{d}$ at which point the myocytes have zero stretch and below which they make no meaningful contraction. This allows the below form

$$
\lambda_{\theta}^{\mathrm{Myo}}=\frac{D}{\alpha_{\mathrm{sf}} D_{d}}
$$


Taking a thin-walled cylinder approximation allows $f_{a}$ to be stated as below.

$$
f_{a}=\frac{2 \tau(D) E_{\max } M_{t}}{D}\left(\frac{D}{\alpha_{\mathrm{sf}} D_{d}}-1\right)
$$

Where $\tau(D)$ is the thickness of the cylinder wall which conserves its cross-sectional area. Which yields the following.

$$
f_{a}=\frac{E_{\max } M_{t}}{D}\left(\frac{D}{\alpha_{\mathrm{sf}} D_{d}}-1\right)\left(-D+\sqrt{D^{2}+4 \tau_{d} D_{d}+4 \tau_{d}}\right)
$$

In deciding a form for $M_{t}$ two factors are taken into account. Firstly, examining Davis' results there appears to be an asymmetry between the duration of the contraction and relaxation phases [2]. Secondly as Davis et al. demonstrates in the same work there is some constant tone present at diasystole which an be represented through some minimum stiffness $E_{\min } \rightarrow\left[0, E_{\max }\right]$. This stimulus can then be modelled through a simple ODE allowing for different kinetics in the contracting and relaxing phase.

$$
\frac{d \hat{M}_{t}}{d t}=\left\{\begin{array}{l}
K_{c}\left(M_{t}-1\right) \omega, \quad \text { Contraction } \\
-K_{r} M_{t} \omega, \text { Relaxation }
\end{array}\right.
$$

These equations can be solved and representative parameters introduced to give the following for $K_{c}$ and $K_{r}$, for derivation see Appendix B.

$$
\begin{gathered}
K_{c}=-\frac{\phi+1}{T \phi} \log \left|\frac{1-M_{\max }}{1-M_{\min }}\right| \\
K_{r}=\frac{\phi+1}{T(\phi-1)} \log \left|\frac{M_{\min }}{M_{\max }}\right|
\end{gathered}
$$

Where $T$ represents the total period of contraction, $\phi$ represents the eccentricity of the contraction, $M_{\max } \& M_{\min }$ are ratios of $E$ at which contraction ends and starts. As a result they change the initial and final rate of contraction by dictating which part of the exponential curve the contraction occurs; as a result $1 \geq M_{\max }>M_{\min } \geq 0$. and $M_{t}$ 
can be scaled to E as below.

$$
M_{t}=E_{\min }+\frac{\hat{M}_{t}-M_{\min }}{M_{\max }-M_{\min }}\left(E_{\max }-E_{\min }\right)
$$

$\phi$, the eccentricity, is the ratio of contraction time and the relaxation time. The consequences on the shape of the $M_{t}$ of varying $\phi$ can be observed below in Figure 3.4.B. Observed lymphatic contractions have significant eccentricities with the contraction time being some order of magnitude smaller than the relaxation time.

Below, in Figure 3.4, the consequences of adjusting the contraction model parameters are explored. A single lymphangion is simulated using the model defined in Section 3.4.4, without inertia, and contracting against an initial trans-mural pressure gradient of $1 \mathrm{cmH}_{2} \mathrm{O}$. In Figure 3.4.A the effect of increasing $M_{\min }$ can be seen on the shape of the relaxation phase of the contraction changes moving with a plateau moving from the end of the relaxation phase to the start as $M_{\min }$ increases. Experimental results indicate that this value is likely to be to the lower end of the simulated range. Figure 3.4.C shows the relatively modest effects of increasing $M_{\max }$ namely a sharpening of the contraction-relaxation transition and an increase in the rate of relaxation. Finally, in Figure 3.4.D the non-linear nature of $E_{\max }$ and the systolic diameter can be seen. Whilst the logarithmic increase in $E_{\max }$ initially produces a logarithmic change in the systolic diameter this quickly plateaus to a constant rate of increase before asymptotically approaching $\alpha_{\mathrm{sf}} D_{d}$. Compared to a modulating tension approach this reduces the sensitivity of the behaviour on $E_{\max }$ or equivalent parameters. 


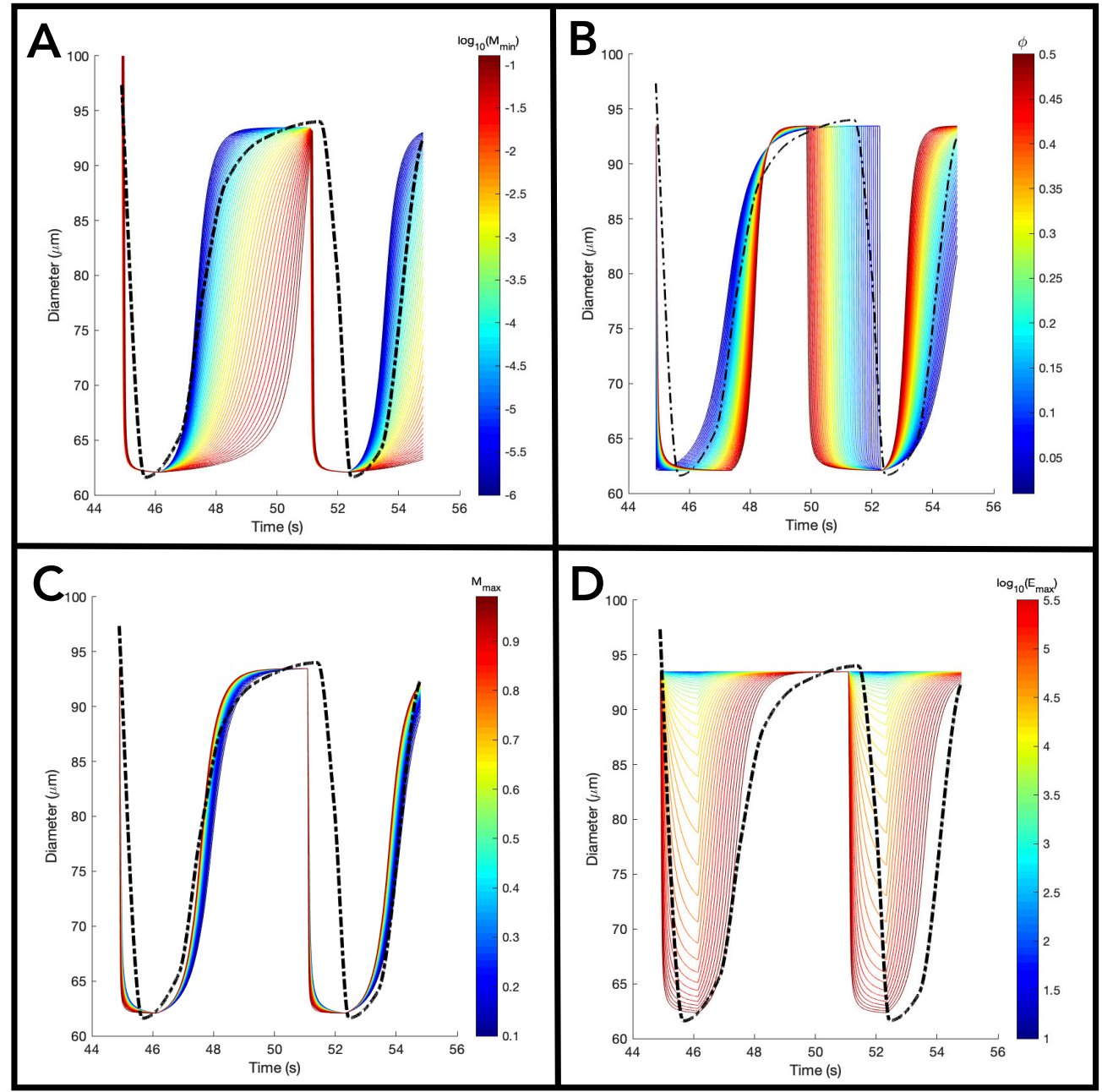

Figure 3.4: The model defined in Section 3.4.4 is used to illustrate the effects of changing the contraction parameters against previously published experimental data. Apart from the parameters under investigation the coefficients are as stated in 4.2. Simulated diameters are shown in colour. Dashed line represents a contraction from Davis et al. [2].

\subsubsection{Increasing Contraction Frequency with Trans-mural Pressure.}

As observed by McHale and Roddie the contraction frequency of lymphangion is a function of the pressure [3]. In a 2015 review Munn described contraction as driven by $\mathrm{Ca}^{2+}$ fluxes from extracellular to intracellular stores[58]. As some of the channels that drive calcium into the cells are stretch-activated. It follows that the contraction frequency increases in response to an increased flux of $\mathrm{Ca}^{2+}$ ions into the cell as a 
response to wall stretch. Contarino and Toro were the first to model this phenomenon in the case of lymphatic contraction through a system of four ODE's based on the FitzHugh-Nagumo model [35]. A simple approach to this would be to assume that $\mathrm{Ca}^{2+}$ ion concentration is an instantaneous function of the circumferential stretch of the lymphangion. Using the constitutive equation in Section 3.3 McHale's pressurefrequency relationship can be converted into a diameter-frequency relationship. As can be seen below in Figure 3.5 this relationship appears to be piecewise linear. This can be simply implemented as a minimum frequency of contraction, $\omega_{\min }$. Which, increases steadily with stretch once a certain threshold, $\lambda_{\text {thes }}$, is exceeded.

$$
\omega\left(\frac{D}{D_{d}}\right)=\left\{\begin{array}{l}
\omega_{\text {min }}, \quad \frac{D}{D_{d}} \leq \lambda_{\text {thes }} \\
\omega_{\text {min }}+\beta_{\text {freq }}, \quad \text { else }
\end{array}\right.
$$

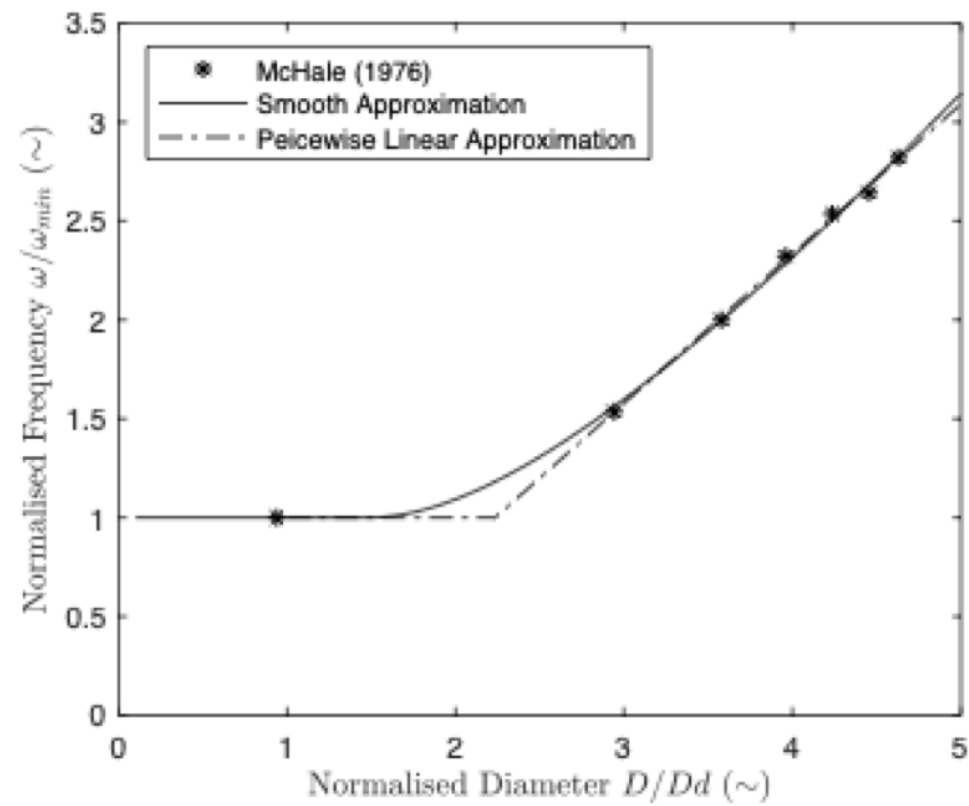

Figure 3.5: The constitutive law in Section 3.3 is used to rescale McHale's pressurefrequency data into pressure-stretch data[3]. Through which a piecewise linear approximation is fitted.

However, this instantaneous frequency would vary largely over a contraction 
cycle as the diameter decreased and increased with pumping. It is assumed that lymphatic muscle cells would have some internal state of $\bar{\omega}$ which would approach $\omega$ in time. In order to give the calculated $\omega$ the character found by McHale, it is instead replaced by $\bar{\omega}$, which is found as a solution to the following ODE.

$$
\frac{d}{d t}\left(\bar{\omega}-\omega\left(\frac{D}{D_{d}}\right)\right)=-K_{\omega}\left(\bar{\omega}-\omega\left(\frac{D}{D_{d}}\right)\right)
$$

With $K_{\omega}$ representing the rate at which $\bar{\omega}$ approaches $\omega$, shown below in Figure 3.6 is an illustrative example of this process calculated from the results presented in Section 4.2.2. The $D / D_{d}$ ratio as a lymphangion pumps against an increasingly adverse pressure gradient is shown as well as the $\bar{\omega}$ value in time for increasing values of $K_{\omega}$. For simplicity, this parameter is taken to have a value of $1 s^{-1}$.
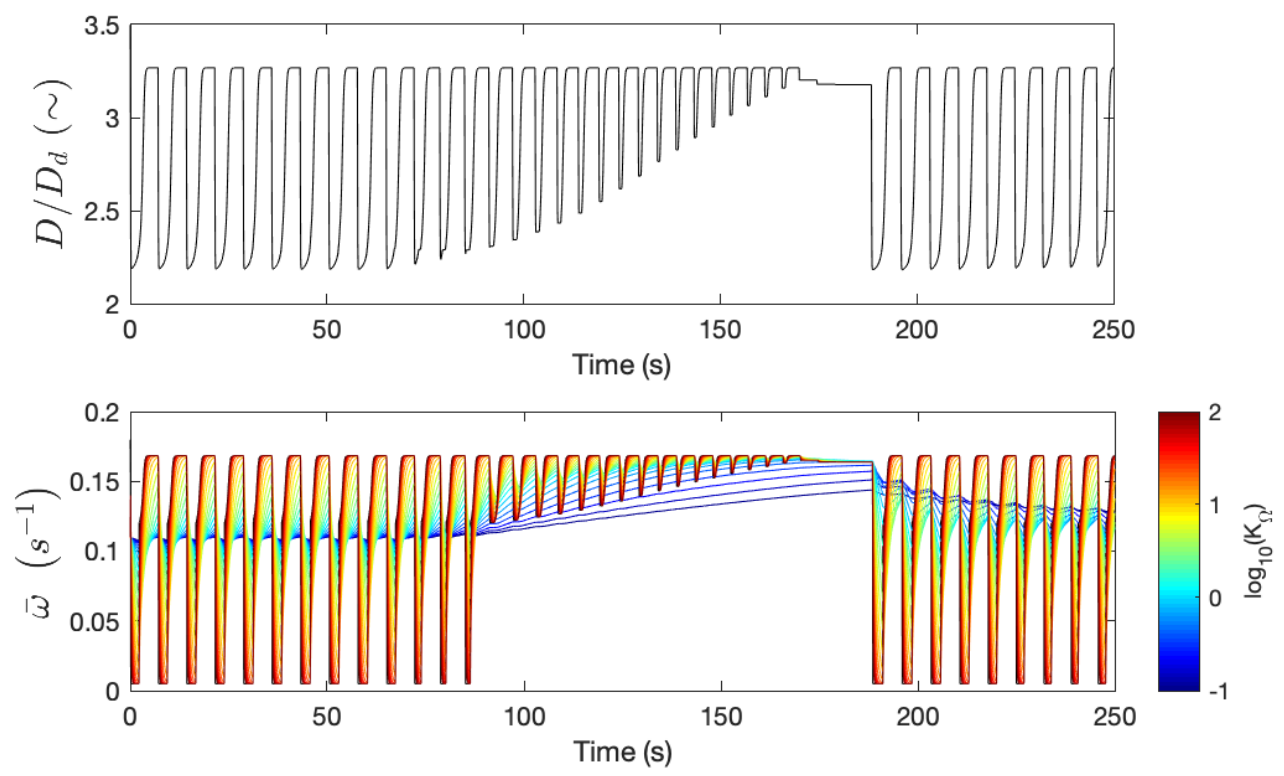

Figure 3.6: Top: the normalised diameter $D / D_{d}$ is shown for a lymphangion pumping against an increasingly adverse pressure gradient. Bottom: the corresponding frequency $\bar{\omega}$ for increasing values of $K_{\omega}$. 


\subsubsection{Discretisation and Assembling a Single Lymphangion}

The discrete system of equations for a lymphangion is constructed as follows. Starting with a resistive element responsible for a pressure drop across a lymphangion, shown below in Figure 3.7. The resistance can simply be found by the Hägen-Poiseuille equation.

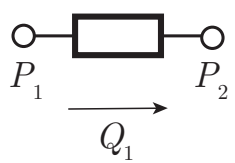

Figure 3.7: The simplest representation of a lymphangion as a Hagen-Poiseuille resistance between two pressures

Equation 3.3 can be discretised for this element at the $n^{\text {th }}$ time step as flows, starting with only the viscous term.

$$
\frac{\pi\left({ }^{n} D\right)^{4}}{128 \eta L}\left[\begin{array}{cc}
1 & -1 \\
-1 & 1
\end{array}\right]\left[\begin{array}{l}
{ }^{n+1} P_{1} \\
{ }^{n+1} P_{2}
\end{array}\right]=\left[\begin{array}{c}
{ }^{n+1} Q_{1} \\
-{ }^{n+1} Q_{1}
\end{array}\right]
$$

This model does not allow for the vessel diameter to change in response to trans-mural pressure as was examined in Chapter 2. There are two considerations when incorporating this effect. Firstly, the change in resistance due to the change in diameter and secondly, the conservation of mass. When the vessel is expanding or contracting the flow entering the vessel will not equal the flow leaving. As in previous models this is resolved by adding a separate conservation of mass equation into the system of equations to be solved [55]. The circuit equivalent model is shown below in Figure 3.8. 


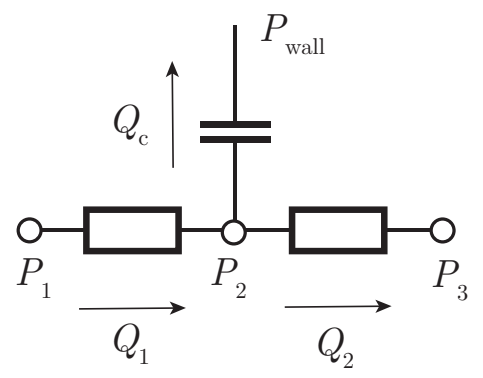

FiguRE 3.8: A representation of a lymphangion with a compliance representing the walls

As was previously done for a lymphangion by Carson [56], a mass balance can be written as follows.

$$
\frac{\pi D L}{2} \frac{\partial D}{\partial t}-Q_{c}=0
$$

This can be restated and discretised at the nth time step using the second-order Taylor expansion of the first order differential operator as the following [56].

$$
\frac{\pi L}{4 \Delta t}\left({ }^{n} D+4{ }^{n-1} D+3{ }^{n-2} D\right){ }^{n+1} D={ }^{n} Q_{c}
$$

The constitutive equation can be introduced through a pressure balance.

$$
{ }^{n} P_{2}-{ }^{n}\left(\frac{\partial P_{\text {wall }}}{\partial D}\right){ }^{n+1} D=P_{\text {wall }}-{ }^{n}\left(\frac{\partial P_{\text {wall }}}{\partial D}\right){ }^{n-1} D
$$

Where $P_{\text {wall }}=P_{\text {ext }}+P_{c}+f_{a}$ and ${ }^{n}\left(\frac{\partial P_{\text {wall }}}{\partial D}\right)$ can be found analytically for the $f_{a}$ and $P_{\text {ext }}$ terms while the $P_{c}$ term can be found numerically. The resultant system is shown below with the following substitutions $r_{\text {flow }}=\frac{64 \eta L}{\pi\left({ }^{n} D\right)^{4}}, k_{\text {comp }}=-{ }^{n}\left(\frac{\partial P_{\text {wall }}}{\partial D}\right), k_{\text {mass }}=$ 
$\frac{\pi L}{4 \Delta t}\left({ }^{n} D+4{ }^{n-1} D+3{ }^{n+1} D\right), f_{\text {comp }}={ }^{n} P_{\text {wall }}-{ }^{n}\left(\frac{\partial P_{\text {wall }}}{\partial D}\right){ }^{n-1} D$

$$
\left[\begin{array}{ccccc}
\frac{1}{r_{\text {flow }}} & -\frac{1}{r_{\text {flow }}} & 0 & 0 & 0 \\
-\frac{1}{r_{\text {flow }}} & 2 \frac{1}{r_{\text {flow }}} & -\frac{1}{r_{\text {flow }}} & 0 & 1 \\
0 & -\frac{1}{r_{\text {flow }}} & \frac{1}{r_{\text {flow }}} & 0 & 0 \\
0 & 0 & 0 & k_{\text {mass }} & 1 \\
0 & 1 & 0 & k_{\text {comp }} & 0
\end{array}\right]\left[\begin{array}{c}
{ }^{n+1} P_{1} \\
{ }^{n+1} P_{2} \\
{ }^{n+1} P_{3} \\
n+1 Q_{c} \\
{ }^{n+1} D
\end{array}\right]=\left[\begin{array}{c}
{ }^{n+1} Q_{1} \\
0 \\
-{ }^{n+1} Q_{2} \\
0 \\
f_{\text {comp }}
\end{array}\right]
$$

The valve can be introduced through adding a variable resistor, where the resistance is directly proportional to $\zeta$ as found in equation 3.4. The equivalent circuit diagram can be seen below in Figure 3.9. The system of equations can be modified as follows, with the introduction of $r_{\text {valve }}=r_{\min }\left(1-{ }^{n} \zeta\right)+r_{\max }{ }^{n} \zeta$. It is important to note that from here on the valve is shown "un-lumped" this is for the sake of the reader so that the equations and pressures are clearly delineated, for this reason $Q_{2}=Q_{3}$ is explicitly stated. This method is functionally equivalent to lumping the valve resistance into the $Q_{2}$ equation and reconstructing ${ }^{n+1} P_{v}$ after solving the system as is shown in Section 3.5. This remains a $0 \mathrm{D}$ lumped model with one pressure node per lymphangion.

$$
\left[\begin{array}{cccccc}
\frac{1}{r_{\text {flow }}} & -\frac{1}{r_{\text {flow }}} & 0 & 0 & 0 & 0 \\
-\frac{1}{r_{\text {flow }}} & \frac{2}{r_{\text {flow }}} & -\frac{1}{r_{\text {flow }}} & 0 & 0 & 1 \\
0 & -\frac{1}{r_{\text {flow }}} & \frac{1}{r_{\text {flow }}}+\frac{1}{r_{\text {valve }}} & -\frac{1}{r_{\text {valve }}} & 0 & 0 \\
0 & 0 & -\frac{1}{r_{\text {valve }}} & \frac{1}{r_{\text {valve }}} & 0 & 0 \\
0 & 0 & 0 & 0 & k_{\text {mass }} & 1 \\
0 & 1 & 0 & 0 & k_{\text {comp }} & 0
\end{array}\right]\left[\begin{array}{l}
{ }^{n+1} P_{1} \\
{ }^{n+1} P_{2} \\
{ }^{n+1} P_{v} \\
{ }^{n+1} P_{3} \\
{ }^{n+1} Q_{c} \\
{ }^{n+1} D
\end{array}\right]=\left[\begin{array}{c}
{ }^{n+1} Q_{1} \\
0 \\
0 \\
-{ }^{n+1} Q_{2} \\
0 \\
f_{\text {comp }}
\end{array}\right]
$$




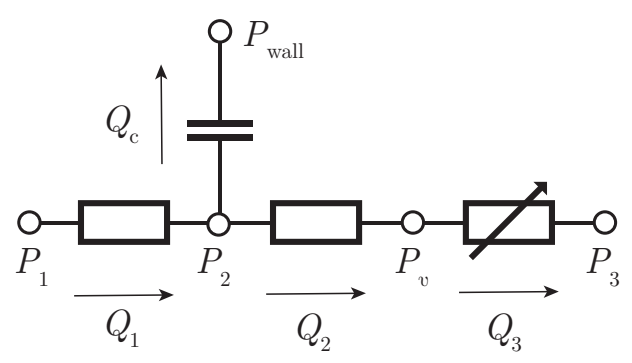

FiguRE 3.9: An equivalent circuit diagram for a lymphangion including a valve

A final modification can be made through the introduction of inertia to the fluid domain. This is achieved by placing an inductor either side of the compliance as can be seen below in Figure 3.10. This only modifies the equations for $Q_{1}$ and $Q_{2}$. Introducing $l_{\text {flow }}=\frac{2 \rho L}{\pi D^{2}}$, to represent the inertial term in equation 3.3 , the equation for $Q_{1}$ can be modified as follows.

$$
{ }^{n+1} P_{1}-{ }^{n+1} P_{2}=\frac{1}{r_{\text {flow }}}{ }^{n+1} Q_{1}+l_{\text {flow }}\left({ }^{n+1} Q_{1}-{ }^{n} Q_{1}\right)
$$

Which can be rearranged to yield.

$$
\frac{1}{r_{\text {flow }}+l_{\text {flow }}}{ }^{n+1} P_{1}-\frac{1}{r_{\text {flow }}+l_{\text {flow }}}{ }^{n+1} P_{2}={ }^{n+1} Q_{1}+\frac{l_{\text {flow }}}{r_{\text {flow }}+l_{\text {flow }}}{ }^{n} Q_{1}
$$




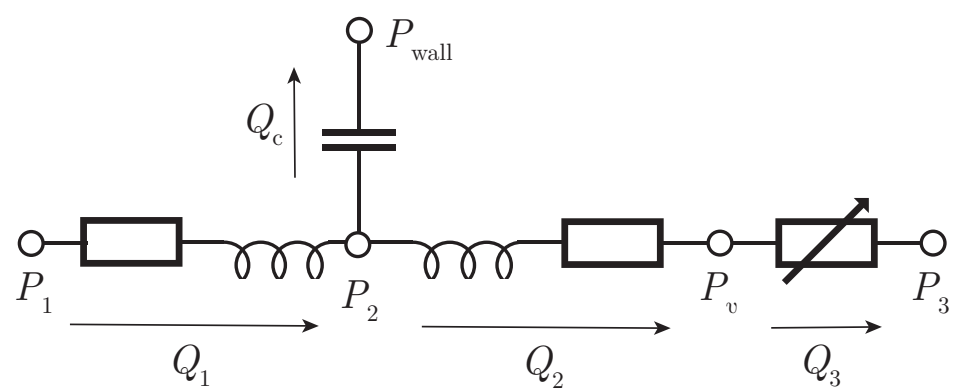

Figure 3.10: The complete equivalent circuit diagram for a lymphangion including inertia

$$
\begin{aligned}
& {\left[\begin{array}{cccccc}
\frac{1}{r_{\text {flow }}+l_{\text {flow }}} & -\frac{1}{r_{\text {flow }}+l_{\text {flow }}} & 0 & 0 & 0 & 0 \\
-\frac{1}{r_{\text {flow }}+l_{\text {flow }}} & \frac{2}{r_{\text {flow }}+l_{\text {flow }}} & -\frac{1}{r_{\text {flow }}+l_{\text {flow }}} & 0 & 0 & 1 \\
0 & -\frac{1}{r_{\text {flow }}+l_{\text {flow }}} & \frac{1}{r_{\text {flow }}+l_{\text {flow }}}+\frac{1}{r_{\text {valve }}} & -\frac{1}{r_{\text {valve }}} & 0 & 0 \\
0 & 0 & -\frac{1}{r_{\text {valve }}} & \frac{1}{r_{\text {valve }}} & 0 & 0 \\
0 & 0 & 0 & 0 & k_{\text {mass }} & 1 \\
0 & 1 & 0 & 0 & k_{\text {comp }} & 0
\end{array}\right]\left[\begin{array}{l}
n+1 P_{1} \\
n+1 P_{2} \\
n+1 P_{v} \\
n+1 P_{3} \\
n+1 Q_{c} \\
n+1 D
\end{array}\right]} \\
& =\left[\begin{array}{c}
{ }^{n+1} Q_{1}-\frac{l_{\text {flow }}}{r_{\text {flow }}+l_{\text {flow }}}{ }^{n} Q_{1} \\
\frac{l_{\text {flow }}}{r_{\text {flow }}+l_{\text {flow }}}{ }^{n} Q_{c} \\
\frac{l_{\text {flow }}}{r_{\text {flow }}+l_{\text {flow }}}{ }^{n} Q_{2} \\
-{ }^{n+1} Q_{2} \\
0 \\
f_{\text {comp }}
\end{array}\right]
\end{aligned}
$$

The system of equations including the inertial term is presented in Eq. 3.31. The other time derivatives used in this work are approximated with the second-order central difference. However, when this was used for the inertial time the scheme was unstable 
and would quickly produce negative diameters. Discretising with a first-order backwards difference yielded stable solutions. One final modification is made to introduce a Newton method with $J$ iterations to improve the estimation of the solution variables within time steps. With ${ }_{j}^{n} X$ representing the $X$ quantity at the $n$th time step and the $j$ th Newton iteration. The following quantities are modified $r_{\text {flow }}=\frac{64 \eta L}{\pi\left({ }_{j}^{n+1} D\right)^{4}}, k_{\text {comp }}=-{ }_{j}^{n+1}\left(\frac{\partial P_{\text {wall }}}{\partial D}\right)$, $k_{\text {mass }}=\frac{\pi L}{4 \Delta t}\left({ }_{J}^{n} D+4{ }_{J}^{n-1} D+3{ }_{j+1}^{n+1} D\right), f_{\text {comp }}={ }_{j}^{n+1} P_{\text {wall }}-{ }_{j}^{n+1}\left(\frac{\partial P_{\text {wall }}}{\partial D}\right){ }_{J}^{n-1} D$. With these new substitutions the system of equations can be modified as shown in Eq. 3.32. When the Newton iterations are complete the time-step is advanced with the assumption that ${ }_{J}^{n} X={ }_{1}^{n+1} X$ for all solution variables.

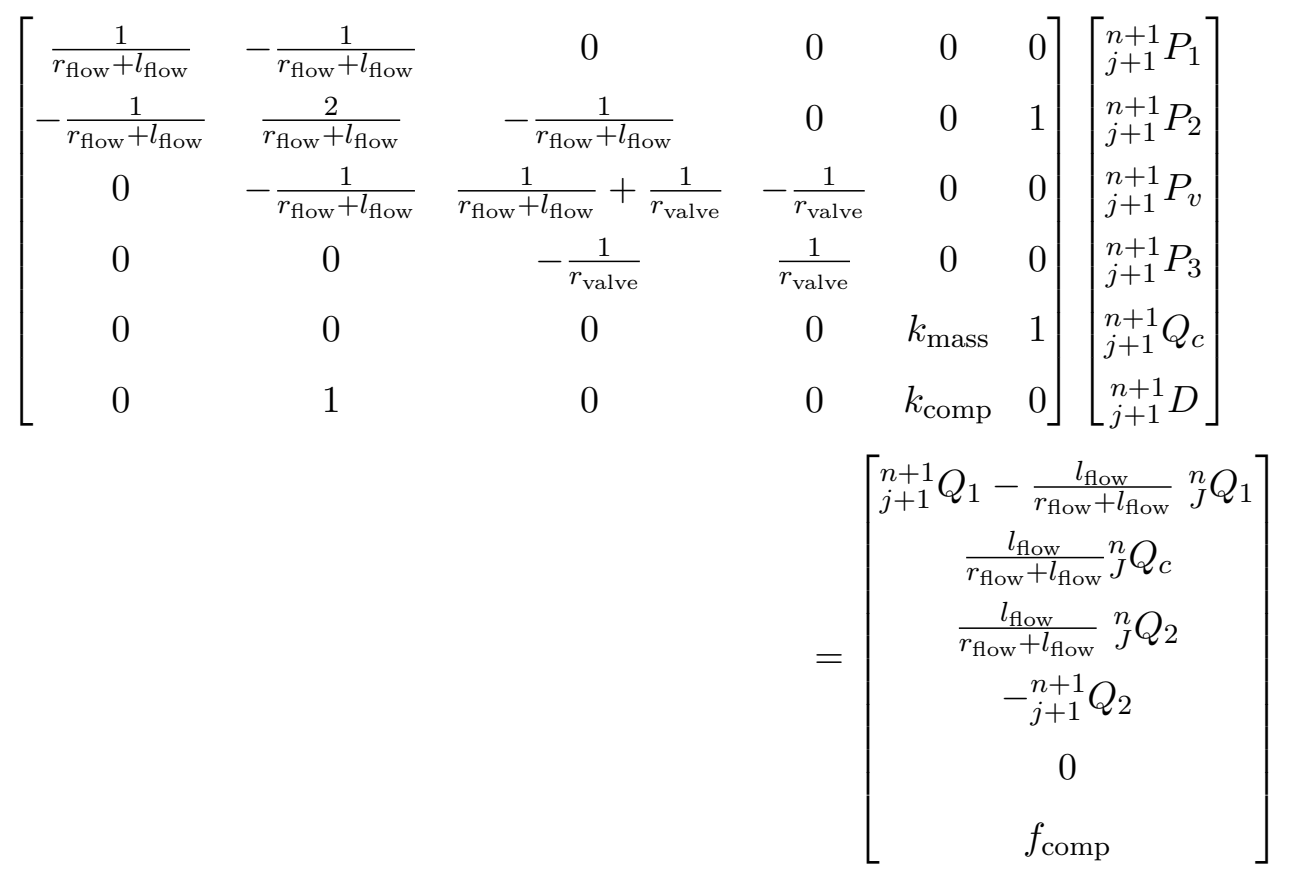

\subsection{Assembling From a Vessel to a Lymphatic Network for the Upper Limb}

The functional element of the collecting lymphatics, the lymphangion, was described in the previous section. By concatenating these elements together vessels can be formed and these vessels arranged into networks. The definition of a lymphangion as a structure with two valves is complicated by the fact that, in a vessel, each lymphangion shares a 
valve with its neighbour. Figure 3.10 shows the lymphangion with only one valve, such that when stacked end to end each lymphangion is bound by two valves. A vessel of a single lymphangion is formed by adding a valve block, as shown below in Figure 3.11. This vessel can be extended by simply adding addition lymphangion elements, as shown in Figure 3.12.

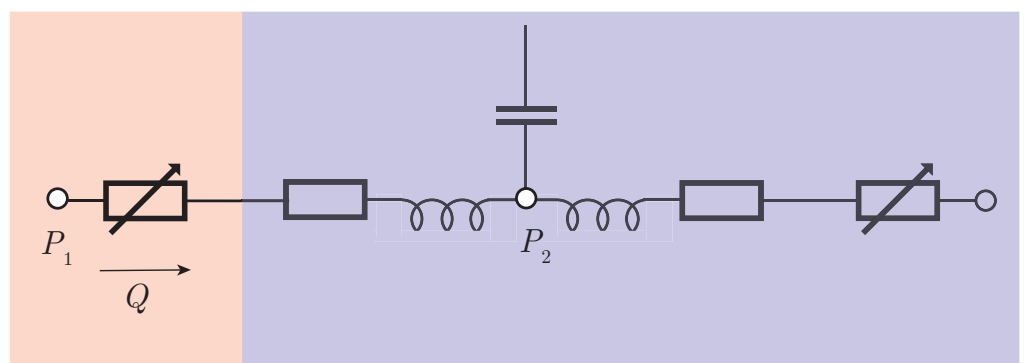

Figure 3.11: The equivalent circuit diagram for a vessel of a single lymphangion. Note the addition of valve block at the left hand side.

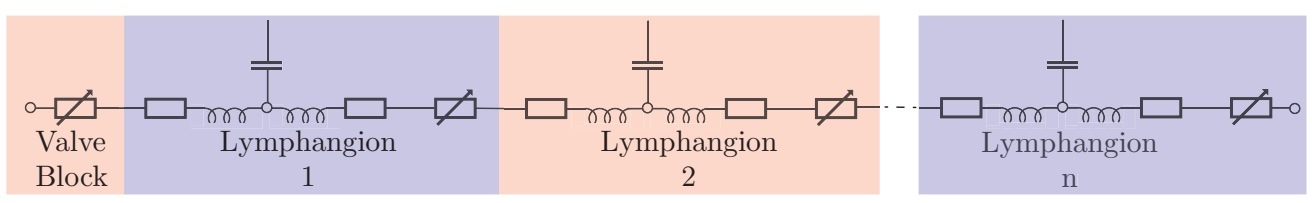

Figure 3.12: A vessel is extended through the addition of more lymphangion elements. Note the lumping of elements leaves one pressure node per lymphangion as discussed in section 3.4.4.

\subsubsection{A Lymphatic Network for the Upper Limb}

Large-scale 3D models of the lymphatic system and the anatomical data necessary to construct them are sparse. Tretyakova et al. based a model on a CGI asset produced by PlasticBoy [59]. This 3D network was produced by an artist and intended for advertising / educational purposes. The network was never intended for quantative study and the number and position of the lymph nodes in the upper limb disagrees with anatomical observations. It remains however, the only published lymphatic network model spanning more than a few lymphangions. ICG and nuclear imaging methods capture a few vessels at poor quality. Suami et al. has developed a method which can produce networks of the 
upper limb with many vessels, by injecting the cadaver limbs with hydrogen peroxide, which allows distended lymphatic vessels to be visualised and injected with contrast [60]. While patent blue is used routinely to visualise lymphatic vessels during surgery it can only be imaged visually and would require the dissection of every lymphatic vessel.

The method Suami et al used is similar to that used by Sappey to produce sketches of the collecting lymphatics of the upper limb in the mid 19th century. Sappey removed the skin from the limb and inverted it, before using peroxide to visualise the vessels and then inject mercurial stains [4]. Sappey's sketches remain more detailed than modern methods and are still used in anatomical textbooks today [61]. In order to produce a 3D network for this work scans of Sappey's sketches were used to reconstruct a 3D network of the superficial lymphatics of the upper limb.

Sappey produced a sketch of the same limb from three different angles allowing the entire network to be seen. The sketch from Planche III of Sappey's Anatomie, physiologie, pathologie des vasisseaux lymphatiques considérés chez l'homme et les vertébrés is reproduced with modification in Figure 3.13.A [4]. Scans (Bibliothéque nationale de France) of each of these figures were manipulated using a series of custom scripts in MATLAB. Each of these three figures was manually traced. The diameter of each sketched lymphatic vessel was measured in pixels in several locations and a linear function found to represent the change of the lymphangion diameter over its length. The trace of fig I. from Planche III is shown overlaid on the original sketch in Figure 3.13.A.

The assumption was made that fig III of Planche III is perpendicular to fig I meaning that these two drawings represented the semi-major and minor axis of the limb. A limb geometry was then constructed as a series of elliptically cross-sectioned frustra, on to which the line elements traced previously, were projected and truncated from the just distal of the shoulder to the wrist. The projected collection of tracings can be seen in Figure 3.13.B. The three sections were connected together by inspection. The network was then manipulated manually to close each connection. The final network is presented in a cylindrical coordinate system in Figure 3.14.A. Suami et al. observed that human lymphatic vessels of the upper limb varied in diameter between $200 \& 500$ 
$\mu m$ [60]. The diameters found from measuring Sappey's sketches were normalised by their mean and rescaled to a mean diameter of $300 \mu \mathrm{m}$, the distribution of diameters can be observed in Figure 3.14.B.

The connectivity is the number of neighbouring vessels which each vessel is connected to, it represents the degree of branching within the network. The distribution of vessel connectivities for the network can be found in Figure 3.14.C. Sappey's sketches include three lymph nodes of which two are included in the final model, for the purposes of this work the lymph nodes were just modelled as connections between vessels.

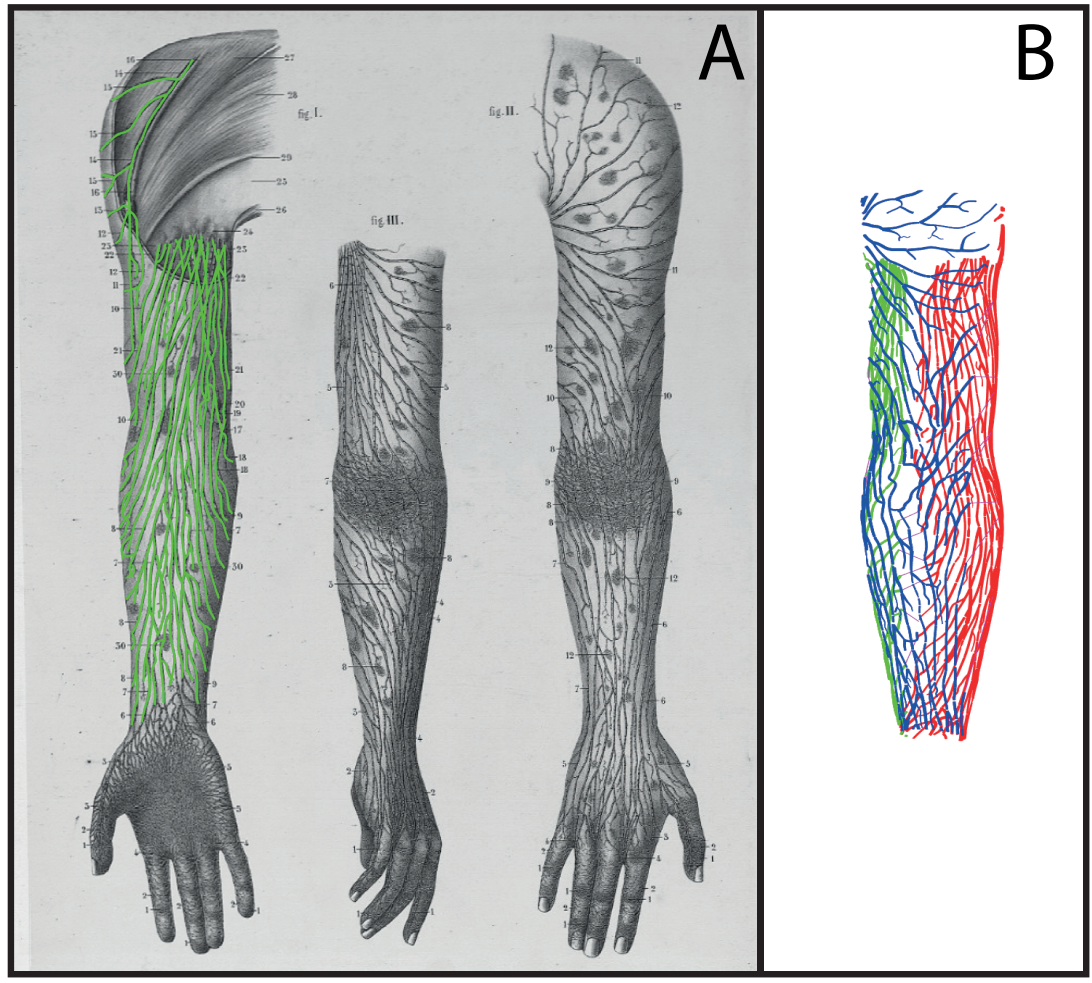

Figure 3.13: A: Planche III from Sappey is shown with the traced vessels overlaid in green [4]. B: The reconstructed network is shown after the traced sections were projected onto a series of fustra 


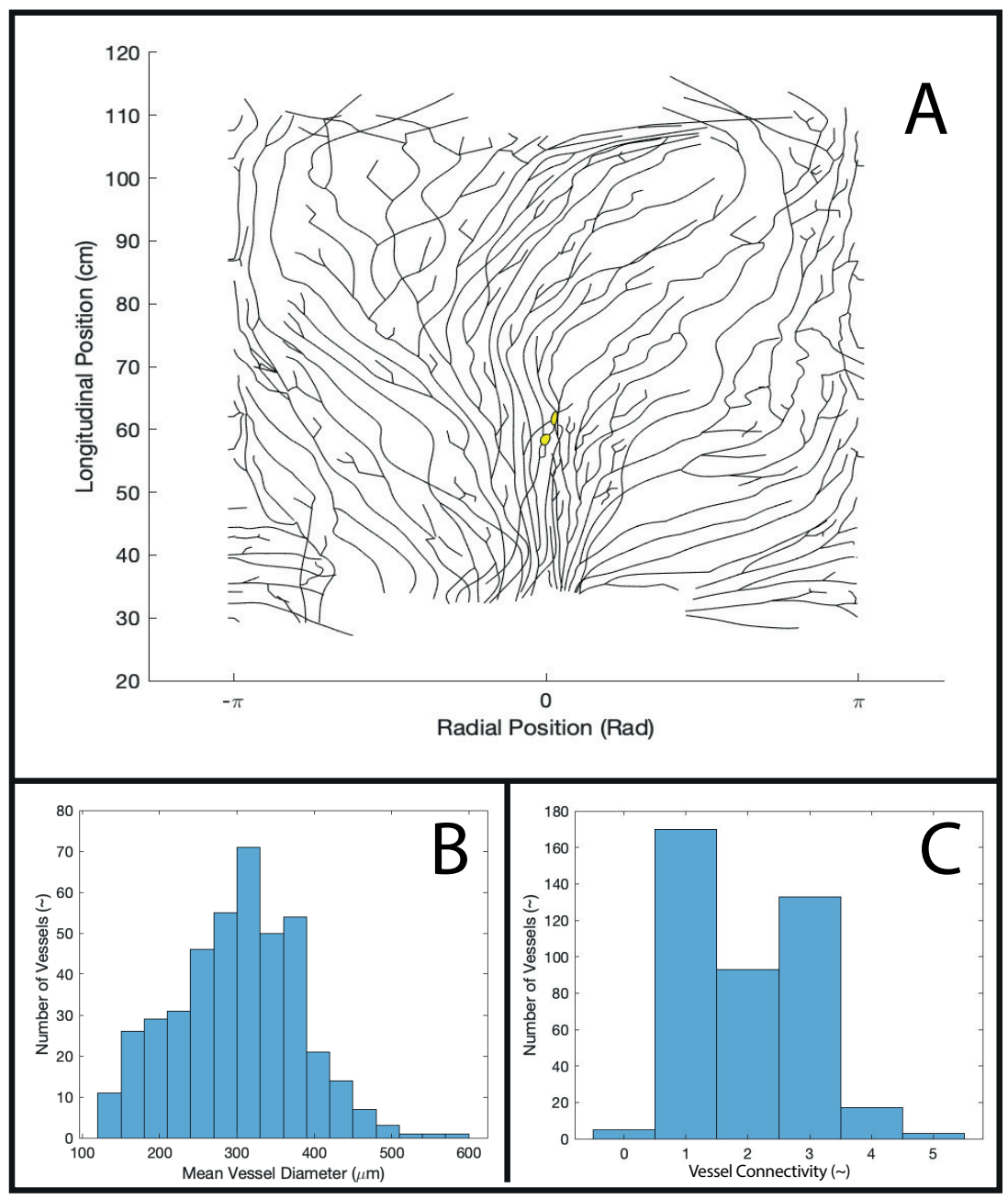

Figure 3.14: A: The final lymphatic limb network is presented in cylindrical form with the axilla located at the bottom of the image and the hand at the top. B: The distribution of vessel diameters in the lymphatic limb network. C: the distribution of vessel connectivities in the lymphatic limb network.

\subsection{Metrics for Exploring Lymphatic Function}

In this section several metrics are designed to assess lymphatic function. The capability of the vessel is the flow rate averaged over a meaningful time scale. The less-idealised nature of the valve means that the lymphangion may experience retrograde flow over the course of a contraction. Therefore, per contraction metrics were designed and then calculated over a contraction cycle. The contraction duration can be found as the time 
between peaks of $f_{a}$, the additional pressure due to contraction. The flow rate, $Q_{2}$ is integrated over the course of a contraction cycle to yield the volume pumped per contraction, $V_{c}$. This allows the average flow rate per contraction to be expressed as $\bar{Q}_{c}=V_{c} / t_{c}$. The work done over a cycle can be calculated by integrating pressure with the respect to volume. For simplicity the integral is performed with respect to time and the volume of the vessel is multiplied by the derivative of pressure such the integral is equivalent to integrating volume with respect to pressure. In order to assess the efficiency of pumping the stroke work of a contraction was calculated as follows.

$$
W_{s}=\int_{t \in t_{c}} \frac{\pi D^{2} L}{4} \frac{d P}{d t} d t
$$

Where $L$ is the length of the lymphangion, $P$ is the pressure and $t$ is the time. The efficiency can then be defined as the volume pumped per unit energy, $\eta_{\text {pump }}=V_{c} / W_{s}$. The period over which this quantity is calculated is shown in Figure 3.15, the closed PV loop can be observed.

If we imagine a rectangular PV loop, then the work done would simply be $W_{s}=\Delta P \Delta V$ so the efficiency would have a scale which would be the reciprocal of the scale of the change in pressure caused by a contraction, $f_{a}$. This reveals the highly efficient nature of these pumps i.e. a lymphangion of $\mathcal{O}\left(f_{a}\right)=1 \mathrm{cmH}_{2} \mathrm{O}$ would pump with $\mathcal{O}\left(\eta_{\text {pump }}\right) \approx 10^{-3} \mathrm{~cm}^{3} /$ erg or $10 \mathrm{~L}$ per Joule. It's important to note that this quantity is not representative of the actual energy used, given the inefficiency of the contraction of the vessels.

In the next chapter these metrics will be used to explore the distribution of efficiencies, their sensitivity to parameter change, and examine how different pressure conditions effect these metrics. 

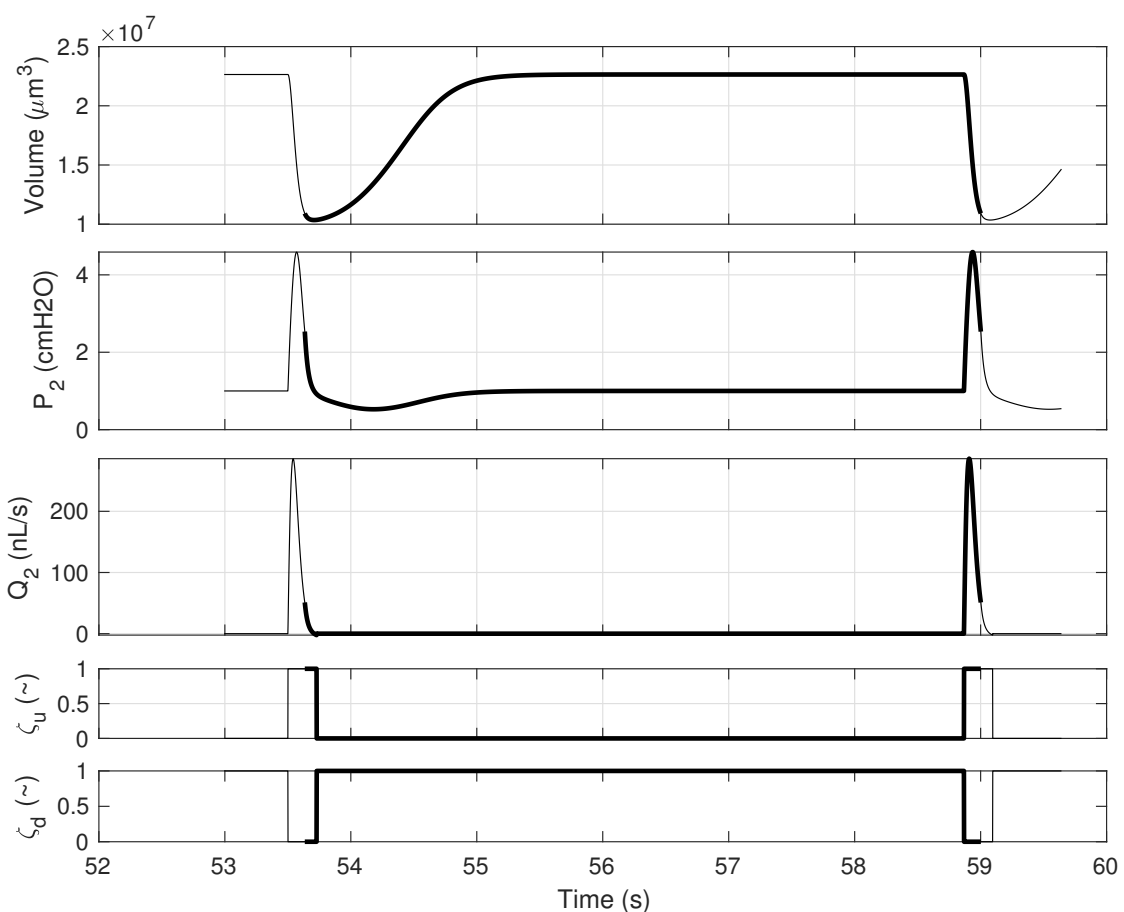

FigURE 3.15: Plots of lymphangion volume, pressure, flow rate and the upstream, $\zeta_{u}$ and downstream $\zeta_{d}$ valve state are shown with a single contraction cycle in bold.

\subsection{Convergence Study and Scheme Stability}

The temporal convergence of the scheme, as proposed in Section 3.5, was assessed by comparing a series of simulations refined by successive halving of the time-step or increase in the Newton iterations. An example case was chosen to represent the finest timestep required. As the pressure gradient against which a lymphangion pumps increases the period over which the valve actions occur increases. When the pressure gradient is greater than the lymphangion can pump against, then there is no change in diameter and no valve actions occur. So temporal convergence is assessed at a point where pumping insufficiency is incipient. Convergence was assessed for each of the solution variables and $f_{a}$, the mean relative absolute error over 90 seconds of simulation was compared to the most refined case is shown in Figure 3.16. 

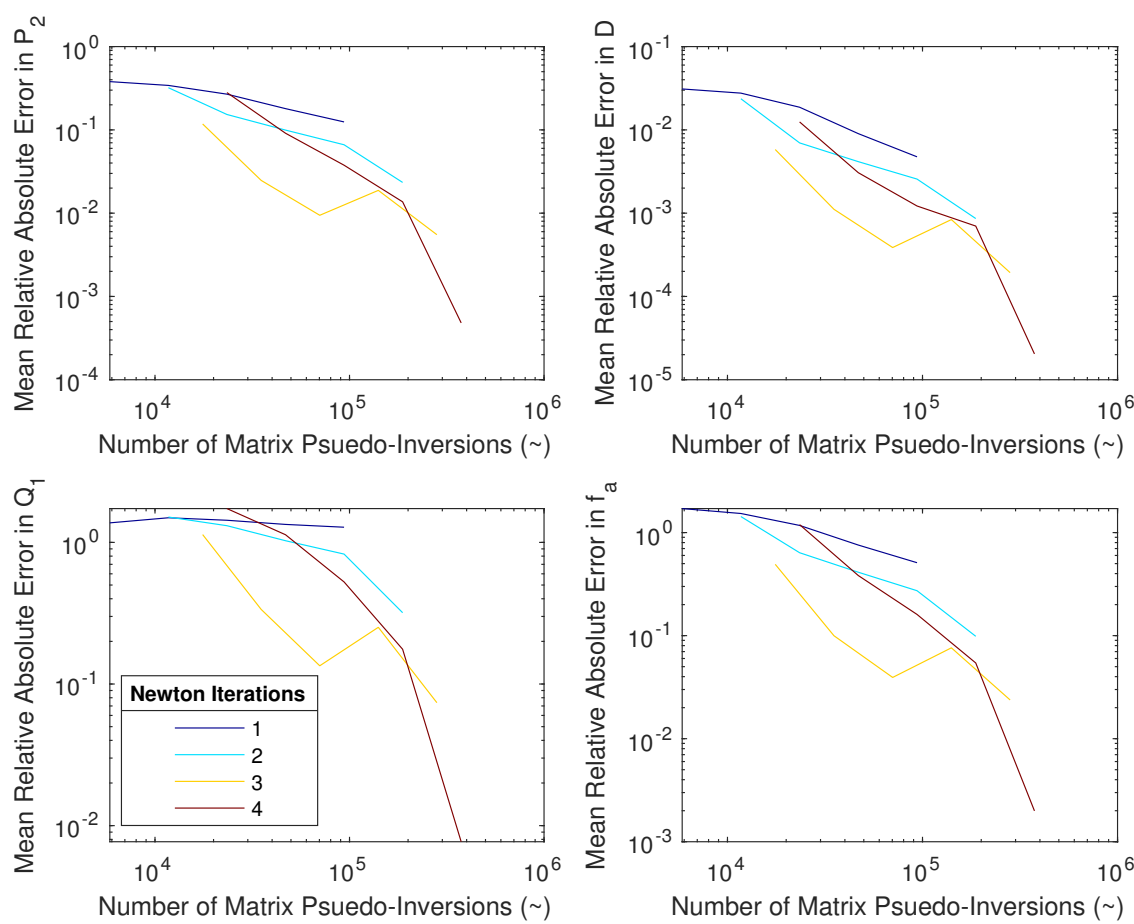

Figure 3.16: The temporal convergence is shown for each of the solution variables and $f_{a}$.

As can be seen convergence is quickly achieved for $D$ and $P_{2}$. For the second finest case, $\delta t=1.28 \times 10^{-3}$ and four Newton iterations per time step, The mean error in $f_{a}$ and $Q_{2}$ is less than one percent. This was deemed acceptable for the analysis being performed and was selected as the time-step.

When the inertial term is omitted and values are used for $k_{v o}$ and $k_{v c}$ are much greater than $10 \mathrm{cmH}_{2} \mathrm{O}^{-1} s^{-1}$ then a "flutter" phenomena is found. Small changes in resistance at the valves effect the formation of trans-valvular and trans-mural pressure gradients. The result is an unstable state where the valve "flutters" between a closing and opening mode. This instability is only resolved by the end of the contraction. This phenomena has not been observed in published works on lymphatic valves and is assumed to be unphysiological. In the presence of the inertial term stabilises the valve state. The consequences of doing so are shown below in Figure 3.17. As can be seen the presence of valve flutter causes large changes in flow rate and diameter but small 
changes in pressure. Whilst the pressure undergoes small oscillations about the valve threshold, the change in resistance causes large changes in flow rate. This phenomena can be avoided by keeping the values for $k_{v o}$ and $k_{v c}$ less than $10 \mathrm{cmH}_{2} \mathrm{O}^{-1} s^{-1}$.

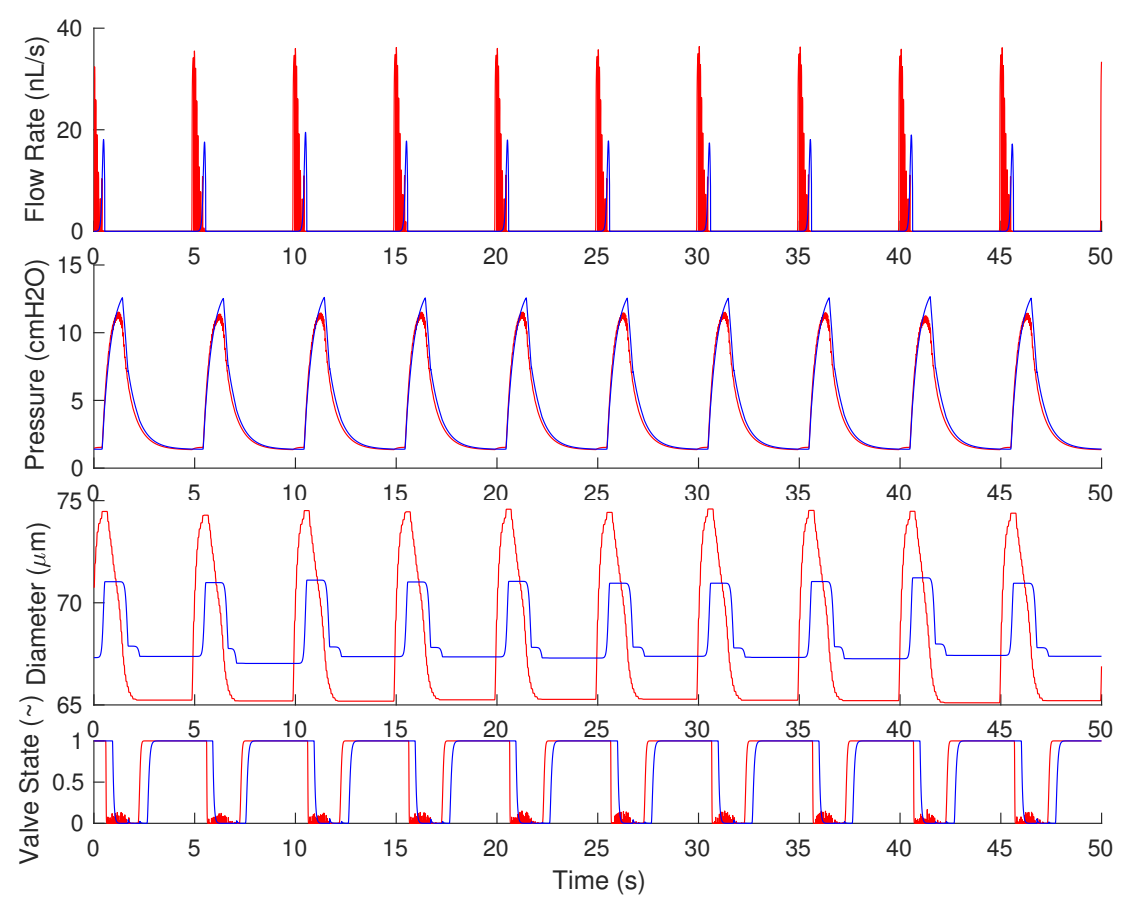

Figure 3.17: The flow rate, diameter, pressure and upstream valve state are shown for a "fluttering" lymphangion. The red line indicates without an inertial term and the blue line with an inertial term. Note the relatively high consequences of flutter on the flow rate and diameter but small consequences on the pressure. 



\section{Chapter 4}

\section{Exploring a 0D Model of the Lymphatic System: From Lymphangion to Limb.}

\subsection{Introduction}

Understanding the flows within the lymphatics of the upper-limb could offer additional insight into the diagnosis and treatment of disease. The lymphatic system is the principle route through which cancer cells metastasis and an understanding of the lymphatic fluid flows at larger scales could allow the predication of metastasis sites [62]. The most common treatments of BCRL of the upper limb involve some form of compression of the limb [63]. These methods involve compressive garment use, multilayer-bandaging and intermittent pneumatic compression [64]. While these methods have a range of efficacies, there is an absence of quantitative justification for the pressures used [65][64]. In this section the model defined in Chapter 3, will be explored to assess the value of this approach and any insights that can be offered into lymphatic function. In particular this section will seek to identify potential avenues for further research that can bridge the gap between lymphatic modelling and therapy for lymphoedema. Results from the 
model will be presented against previously published experimental data and studied to assess the effect of trans-mural and trans-valvular pressures on the mean flow rate $\bar{Q}_{c}$ and the efficiency $\eta_{\text {pump }}$ on a chain of 10 lymphangions and the network of the upper limb.

\subsection{Methods}

In order to reduce the number of parameters under consideration the fit for the valve bias model found in section 3.2 is assumed correct and the following constants are assumed: Lymph density $\rho=1 \mathrm{~g} \mathrm{~cm}^{-3}$, lymph viscosity $\eta=1 \mathrm{cP}$ and the constitutive law defined pressure $P_{d}=35$ dynes $\mathrm{cm}^{-2}$

\subsubsection{Sensitivity Analysis}

In order to assess the sensitivity of the model to variation in parameters a global variance sensitivity analysis was performed using the SAFE toolbox [66]. Briefly, Sobol indices study a function which relates the variance in the input parameters to the variance of the output. This function can be decomposed into a polynomial series of increasing orders of the parameter sets. By calculating the first order terms and the total variances this function can be represented as the "main effects", the first order relationship between parameter variance and output variance and the "total effects" the sum of all remaining terms in which that parameter appears. The quality of the estimation of these quantities is directly related to the number of samples evaluated, and every sample needs to be evaluated multiple times. The quality of the estimation of the Sobol indices can be assessed through "bootstrapping" which allows confidence intervals of the Sobol indices to be evaluated without additional samples [67][66].

A single lymphangion with a trans-mural pressure of $1 \mathrm{cmH}_{2} \mathrm{O}$ was simulated 
for 60 seconds. The parameters and the ranges considered are shown in Table 4.1; together they form a 12D parameter space, this space was chosen to represent the computational limits of the model. A quasi-random uniform distribution was sampled through the parameter space using the latin hypercube method. The computational expense of calculating Sobol indices can be greatly reduced if the samples are triplicated such that only certain parameters change between the triplets as described by Saltelli et al [68][66]. Sobol indices were calculated from 10,000 samples distributed in the ranges shown below in Table 4.1. There were 110000 evaluations for 12 parameters with 1000 samples used for bootstrapping.

\begin{tabular}{r|c|c|c|c} 
Symbol & Description & Lower Bound & Upper Bound & Units \\
\hline$K_{o}$ & Valve opening rate & 0.01 & 10 & $s^{-1}$ \\
$K_{c}$ & Valve closing rate & 0.01 & 10 & $s^{-1}$ \\
$\phi$ & Eccentricity of contraction & $10^{-3}$ & $1-10^{-3}$ & $\sim$ \\
$E_{\max }$ & Maximum value of $M_{t}$ & 10 & 1000 & $\mathrm{cmH}_{2} \mathrm{O}$ \\
$E_{\min }$ & Minimum value of $M_{t}$ & 0.01 & 10 & $\mathrm{cmH}_{2} \mathrm{O}$ \\
$M_{\max }$ & Maximum value of $\hat{M}_{t}$ & 0.5 & 1 & $\sim$ \\
$M_{\min }$ & Minimum value of $\hat{M}_{t}$ & $10^{-16}$ & 0.5 & $\sim$ \\
$\alpha_{\mathrm{sf}}$ & $D$ & 3 & $\sim$ \\
$\omega_{\min }$ & Minimum contraction frequency & $10^{-4}$ & 3 & $s^{-1}$ \\
$\lambda_{\text {thres }}$ & $D$ ratio at which $f_{a}=0$ & 0 & 3 & $\sim$ \\
$\beta_{\text {freq }}$ & Rate of frequency increase after contraction & 0 & 4 & $s^{-1}$ \\
\hline
\end{tabular}

TABLE 4.1: A table showing the range of parameters used in the sensitivity analysis.

\subsubsection{Parameter Estimation}

Davis et al. performed experiments which elucidate the valve gating of a lymphangion pumping between two pressure reservoirs. However, these experiments also offer a holistic perspective on lymphatic pumping and contain relationships between diameter, pressure which lymphatic modelling should seek to recapitulate. Detailed results are presented for only one lymphangion has been presented pumping against a pressure gradient, which increases in time. This data has been used in the development of lymphangion models [2][40][35]. An attempt was made to fit the model described in Chapter 3 to the data presented by Davis et al.[2]. 
Initial attempts, made using classical methods, were an attempt to find the parameter set which most closely agreed with experimental results. A particle swarm method was used. Whereby, a series of particles are distributed over the parameter space and they move with each iteration based on two factors a momentum in the direction which minimises the objective function and a social factor attracting them toward other particles with lower objective functions. This method was computationally expensive as complete simulations had to be performed for each particle and the approach to the minima was slow. The sensitivity of the social and momentum meant particles could "skip" over local minima if the social factor was too high or fail to explore the parameter space if the momentum factor was too high. In order to address this; several other methods including cuckoo search, co-variant matrix adaptation by evolutionary strategy, genetic algorithm, differential evolution and gradient descent were also attempted with similar results. Finally, a Bayesian inference method was attempted using the ensemble Kálmán filter method [69]. A step by step explanation of the method is included in Appendix A. Briefly, a series of particles are distributed according to a Gaussian distribution throughout a transformation of the state space, including the parameters, such that the state values are bounded but the transformation isn't. These particles are then evaluated and the distribution of objective function valves is observed as a posterior, which is then used to correct the variance of the particles and the state is propagated. This method was much cheaper, computationally, as the simulation was run once for each particle. However, as the particles converged to a solution the valve state variance would collapse as all particles would be opened or closed. An attempt to avoid this was made by removing the valve states from the state and imposing them a priori. However, this did not yield good fits. In order to proceed the model was fitted manually by inspection. The resulting parameter values are presented below in Table 4.2 and will be used unless otherwise specified for the remainder of this work. 


\begin{tabular}{r|c|c|c} 
Symbol & Description & Value & Units \\
\hline$K_{o}$ & Valve opening rate & 1 & $s^{-1}$ \\
$K_{c}$ & Valve closing rate & 1 & $s^{-1}$ \\
$\phi$ & Eccentricity of contraction & 0.02 & $\sim$ \\
$E_{\max }$ & Maximum value of $M_{t}$ & 510 & $\mathrm{cmH}_{2} \mathrm{O}$ \\
$E_{\min }$ & Minimum value of $M_{t}$ & 0.1 & $\mathrm{cmH}_{2} \mathrm{O}$ \\
$M_{\max }$ & Maximum value of $\hat{M}_{t}$ & 0.75 & $\sim$ \\
$M_{\min }$ & Minimum value of $\hat{M}_{t}$ & $1 \times 10^{-6}$ & $\sim$ \\
$\alpha_{\mathrm{sf}}$ & $D$ & 2.15 & $\sim$ \\
$\omega_{\min }$ & Minimum contraction frequency & 0.05 & $s^{-1}$ \\
$\lambda_{\text {thres }}$ & $\frac{D}{D_{d}}$ threshold for increase in frequency & 2.7 & $\sim$ \\
$\beta_{\text {freq }}$ & Rate of frequency increase after contraction & 0.25 & $s^{-1}$ \\
\hline
\end{tabular}

TABLE 4.2: A table of the values found by manually fitting the model against the data from Davis et al.[2]

\subsubsection{Lymphangion Chain Study.}

A chain of 10 lymphangions, with a defined diameter, $D_{d}=30 \mu \mathrm{m}$ and a length of $3 \mathrm{~cm}$, was assembled as described in section 3.5. The valve block that preceded the first lymphangion was designated as an inlet and prescribed a pressure, $P_{i n}$, and the last lymphangion was designated as an outlet and prescribed a pressure, $P_{\text {out }}$. A steady-state flow study, without the valve or contraction terms, was iterated with a Newton-Rhapson scheme until the difference in diameter was less than $10^{-6} \mu \mathrm{m}$. The found resultant pressures and diameters were used to initialise the simulation, with the valves set to open and the contraction state, $\hat{M}_{t}$ set to $E_{\min }$. The solution variables were allowed to evolve for a period of 600 seconds and the contractions were analysed to find the metrics defined in section 3.6. $P_{\text {in }}$ and $P_{\text {out }}$ were varied between 1 and $5 \mathrm{cmH}_{2} \mathrm{O}$ to produce a range of 20 pressure loading conditions. This process was repeated without the inertia term. A second study was performed where the valve properties $K_{o}, K_{c}$ and the valve bias relationship were adjusted for the chain against a $1 \mathrm{cmH}_{2} \mathrm{O}$ to explore the effects of less-idealised valve models. 


\subsubsection{Limb Network Study.}

The limb network as described in section 3.5.1, was populated with lymphangions. Little data exists on the lymphangion spacing within the lymphatic networks of the upper limb. A spacing of $1 \mathrm{~cm}$ was assumed based on an approximation of an aspect ratio previously assumed [43]. The vessels were populated with lymphangions, at vessel junctions the valve block was absent to prevent multiple valves being placed together without a compliant section between them. The resultant network consisted of 17706 lymphangions. Lymphatic branches which terminate at the proximal end of the limb are labelled as inlets all other terminations are labelled as outlets. Figure 4.1 shows the cylindrical representation of the limb as in Figure 3.14.A but with the inlet and outlets marked.

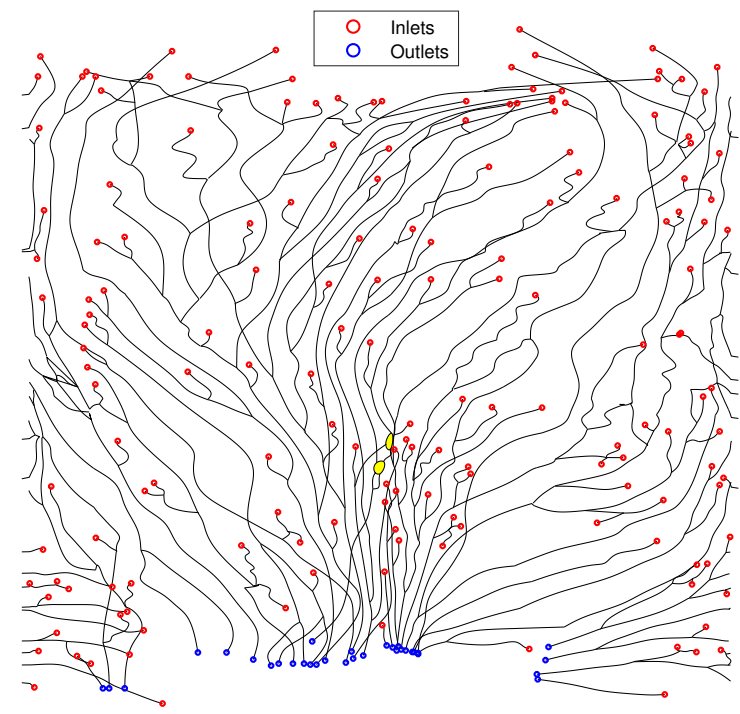

Figure 4.1: A cylindrical representation of the limb network model with the boundaries marked. 


\subsection{Results}

\subsubsection{Sensitivity Analysis}

The results of the sensitivity analysis can be seen below in Figure 4.2. The left plot shows the estimated Sobol indices for the average flow rate, $\bar{Q}_{c}$. As can be seen the uncertainty in the derived indices is high enough to only allow qualitative statements of the sensitivity of parameters. There are several parameters for which the error exceeds the value. However, it's clear that $\omega_{\min }, \beta_{\text {freq }}, E_{\min }$ and $M_{\max }$ are largely insignificant. Though it is important to note that this may be a reflection of the case selected for analysis. In the absence of a trans-valvular pressure gradient any $M_{\max }$ would be sufficient to cause pumping and the limit of the degree of contraction would be dictated by $\alpha_{\mathrm{sf}}$ to which the model is sensitive. $\alpha_{\mathrm{sf}}$ also shows the highest discrepancy between the first order and total Sobol indices for average flow rate indicating that relative to other parameters its largest effects are caused by interactions with other parameters. The valve parameters $K_{o}$ and $K_{c}$ are the parameters with the largest first order effects and are likely to have the largest total sensitivity. $E_{\max }$ appears to have insignificant first order sensitivity. However, given its effect on the shape of contraction it is to be expected that in combination with other parameters it can cause a significant change in $\bar{Q}_{c}$. A similar effect is noted for the eccentricity of contraction, $\phi$.

The sensitivity analysis of the pumping efficiency is less informative. This is not unsurprising given that the volume pumped per unit energy will be dictated by the regime of the constitutive law traversed during contraction. It's clear the variance in efficiency is dominated by the interaction between parameters. Narrowing the parameter space could allow for a more informative sensitivity analysis. Experimental data that constrained this space may allow more insight into the relationship between efficiency and the parameters. 

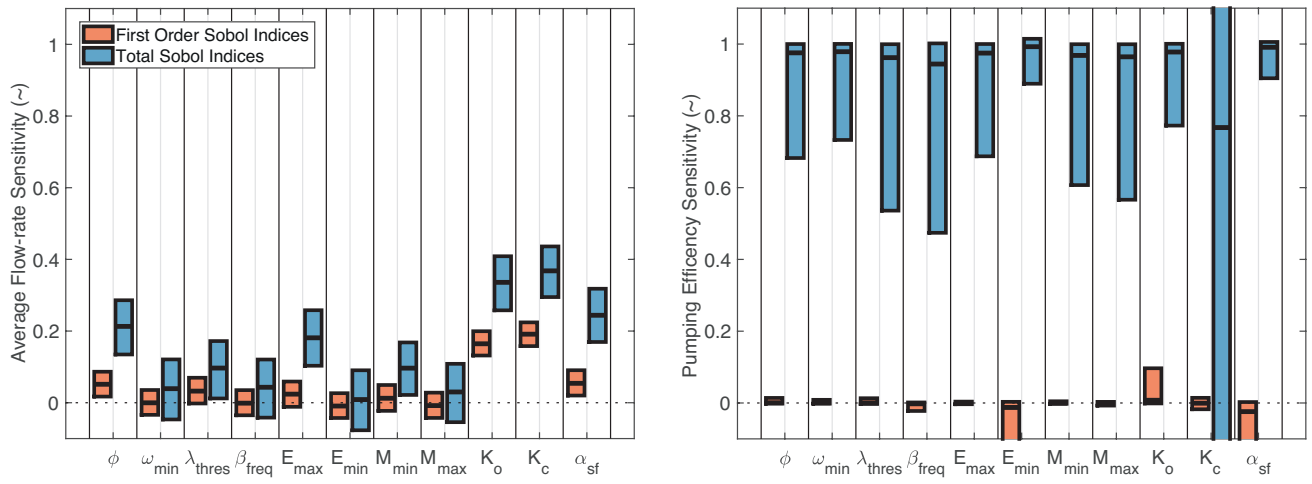

Figure 4.2: Left: the first order and total sensitivity index of each parameter on average flow rate, $\bar{Q}_{c}$, is shown with the $95 \%$ confidence interval. Right: the first order and total sensitivity index of each parameter on pumping efficiency, $\eta_{\text {pump }}$, is shown with the $95 \%$ confidence interval.

\subsubsection{Coordination of Lymphatic Pumping}

The proposed stretch dependent frequency of contraction model allows lymphangions to coordinate their pumping. Vessels which pump surplus to requirement will deplete their volume, slowing their contraction frequency and vessels which are accumulating fluid will increase their rate of contraction. While previously published network scale models relied on prescribing a phase difference between adjacent lymphangions the formation of phase differences in this model can be observed to form spontaneously. Shown below, in Figure 4.3 a chain of 10 lymphangions initially pumping simultaneously, can be seen to form a train of contractions over time. The first contraction occurs at slightly different frequencies, due to the difference in stretch caused by the pressure gradient along the chain. As the pumping is established within the lymphangions the average contraction frequency falls due to a fall in the average stretch because of contraction. Over time a semi-periodic pattern of contraction forms, with a unimodal distribution of contraction durations. The establishment of this pattern can be seen as an initialisation step and measurements can be made when this process is complete. 


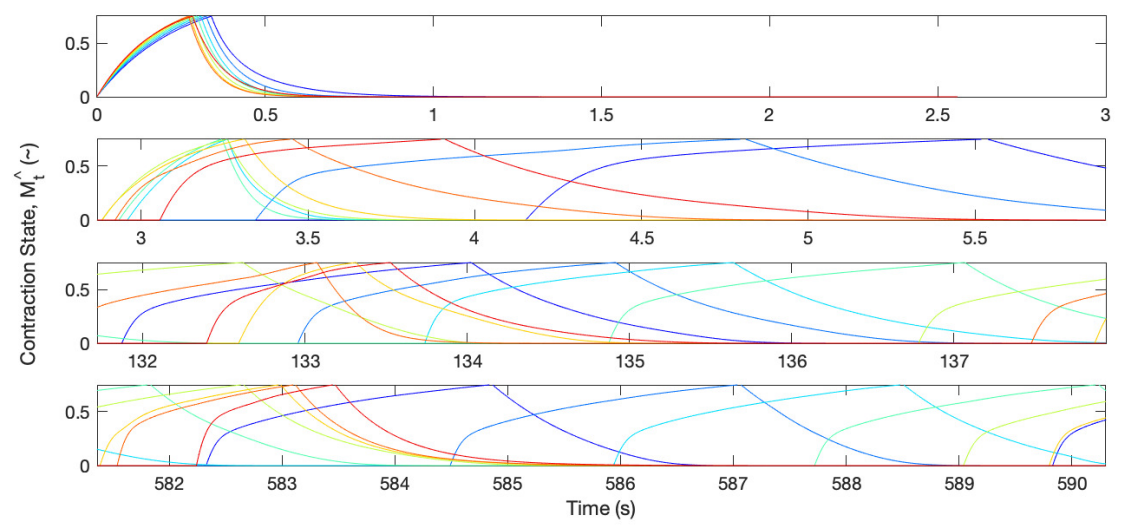

Figure 4.3: The contraction state, $\hat{M}_{t}$, of a chain of 10 lymphangions pumping against a $1 \mathrm{cmH}_{2} \mathrm{O}$ pressure difference is shown at four different time points over 10 minutes of simulation. Coloured by distance from the inlet, blue, to the outlet, red.
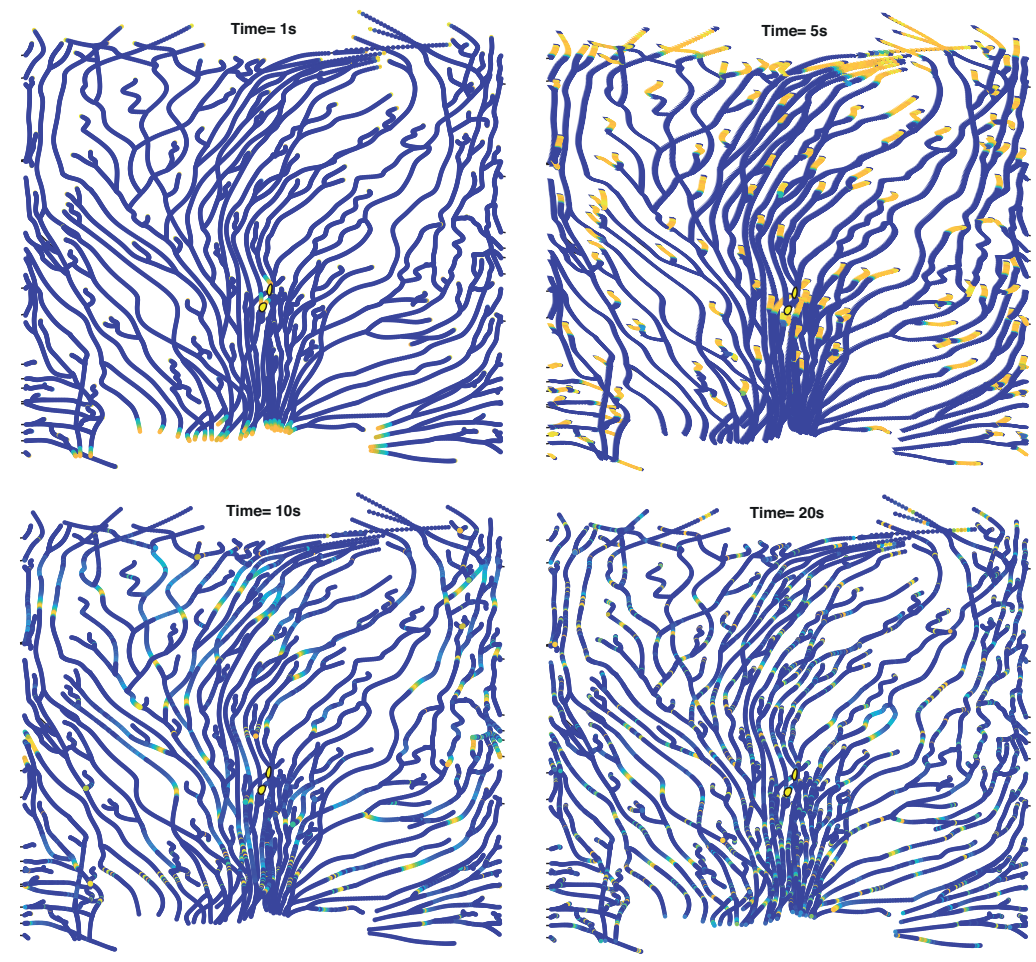

FiguRE 4.4: Four cylindrical representations of the lymphatic limb network coloured from blue to yellow proportional to the contraction state $\hat{M}_{t}$ showing the initialisation of contractions against a $1 \mathrm{~cm} \mathrm{H}_{2} \mathrm{O}$ pressure difference. 
This effect is also observed in a network of lymphangions, see Figure 4.4. Initially contractions occur fastest at the outlets, as time progresses chains of contractions occur and the average frequency drops. Unlike the chain case, these trains of contractions interact at confluences and the resultant behaviour is complex. This complicates establishing the point at which a stable pattern has formed. The following assumption was taken, the contraction pattern is established when the distribution of contraction times is constant. Figure 4.5 shows the probability density of contraction duration over time. As can be seen the initial distribution is unimodal. As pumping is established, the range of contraction durations increases and the mean contraction duration reduces. Eventually, a stable bi-modal distribution forms with two groups of lymphangions. The first set pumping just above $f_{\min }$ and the second set pumping at approximately $1 \mathrm{~Hz}$. This behaviour is indicative of an insufficiency in the network. Where lymphangions are unable to prevent accumulation of fluid, their diameter and contraction frequency will increase. As can be seen in Figure 4.6 and 4.7, this phenomenon is principally observed at confluences, a hypothesis is addressed in section 4.4.1.

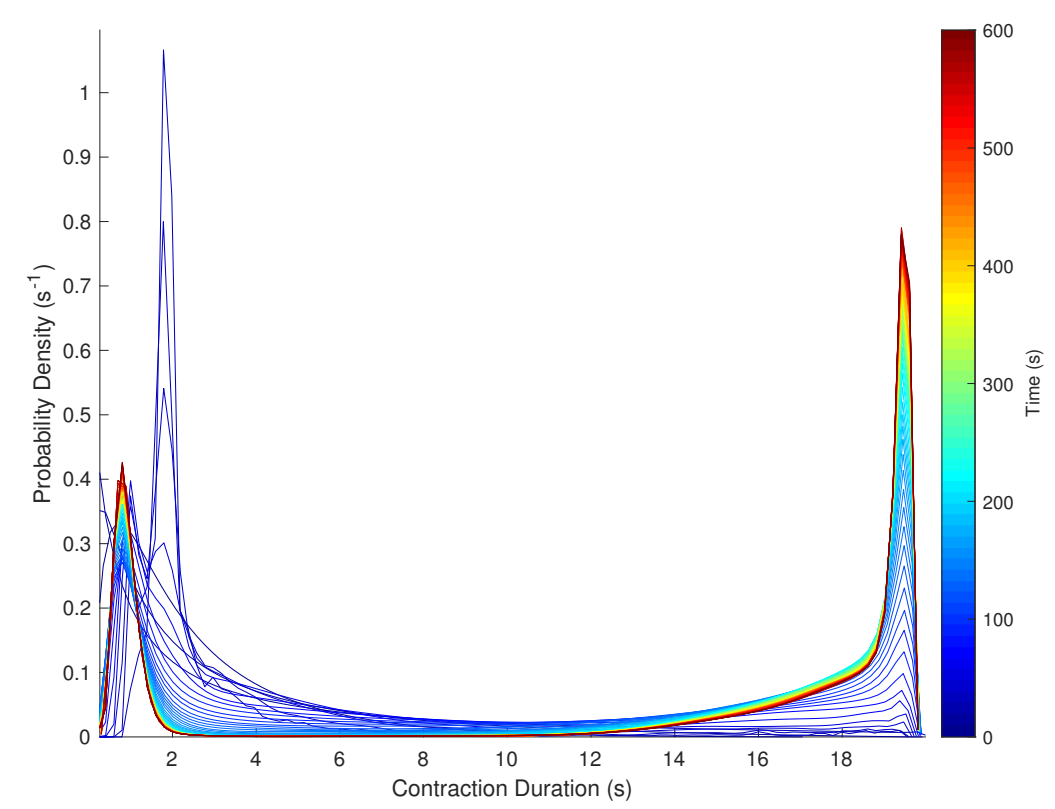

Figure 4.5: The probability density of contraction duration of a lymphatic limb network pumping against a $1 \mathrm{cmH}_{2} \mathrm{O}$ pressure difference over 600 seconds. 


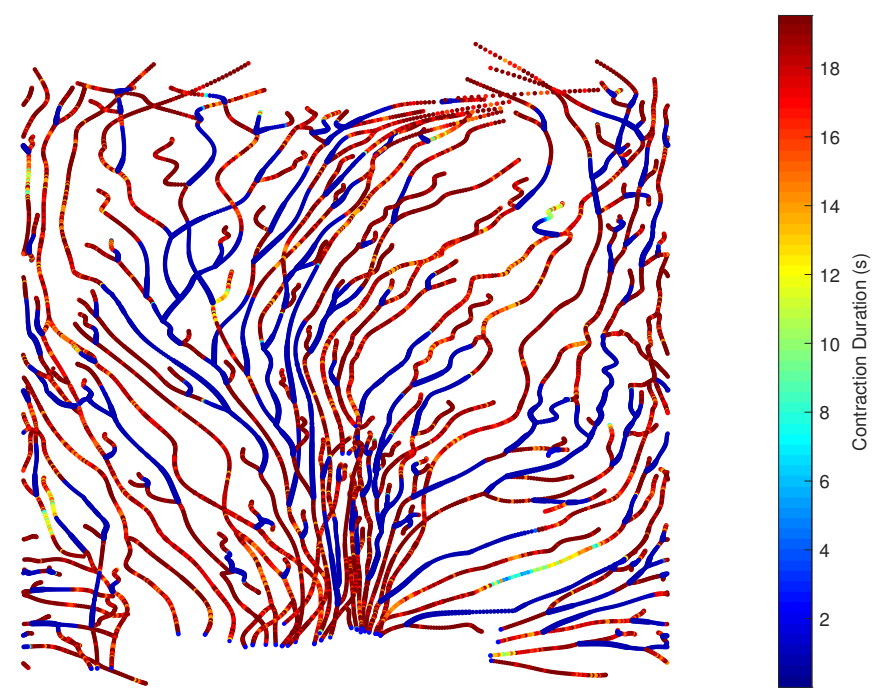

FiguRE 4.6: A radial representation of the limb network is shown coloured by the final contraction duration of 600 second simulation pumping against a $1 \mathrm{cmH}_{2} \mathrm{O}$ pressure difference 


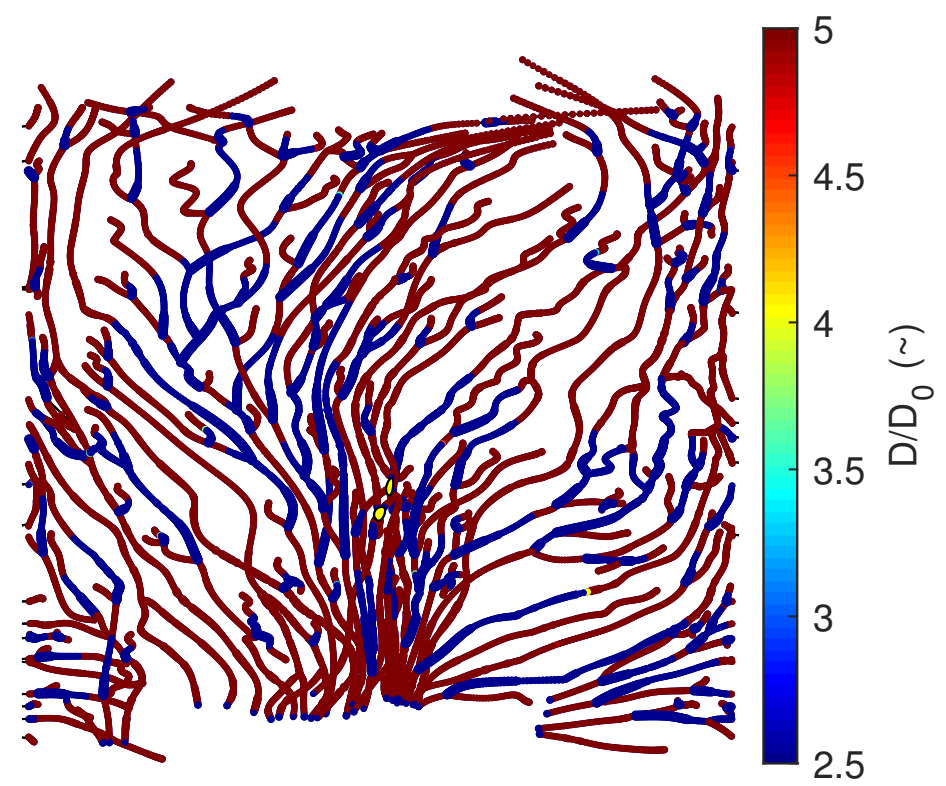

FiguRE 4.7: A radial representation of the limb network is shown coloured by the average $D / D_{0}$ ratio for the final contraction cycle of a 600 second simulation pumping against a $1 \mathrm{cmH}_{2} \mathrm{O}$ pressure difference

\subsubsection{The Effects of Inertia}

Standard reasoning would lead one to omit inertial effects from a model such as this given the low Reynolds number of the flow and small nature of the vessels. As observed by Davis et al. the valve action occurs over sub-second time periods. The small latency in the contraction cycles allow several lymphangions to have valve states within one period of each other which could form a contiguous volume spanning multiple lymphangions. This volume would to be bought to a stop over a time period otherwise uncharacteristic for this system. If the Reynolds number is calculated using the vessel diameter and the average velocity then it will be very low and this justification for omitting inertial effects is well founded. However, as seen in Figure 4.2 the major drive of average flow rate is the valve closing and opening rate. The open-bias nature of the valves mean that these valve motions occur when a pressure gradient supports flow through the 
valve. Small changes in apparent valve closing and opening rates - which are driven by trans-valvular pressure - could have large effects on the average flow rate. The scalings used to determine Reynolds numbers are not appropriate for judging whether inertia in the fluid domain effects valve timing. While these phenomena may be insignificant in a single lymphangion they could produce large changes in the flow rate of a chain of lymphangions.

Figure 4.8 shows the pressure and diameter curves of a single lymphangion with and without an inertial term, pumping with no inlet-outlet pressure difference. Over a 200 second simulation the difference in the pressure and diameter curves is insubstantial and largely due to timing. The average relative error over the entire simulation period is also shown on each plot.
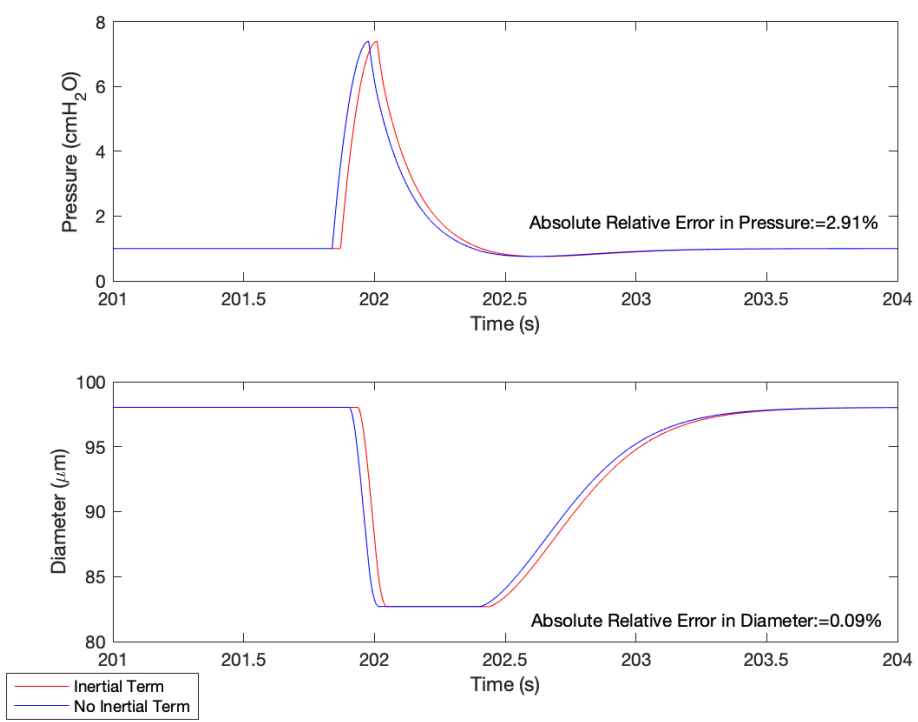

Figure 4.8: Pressure and diameter traces showing the consequences of omitting the inertial term is shown for a single lymphangion after 200 seconds of simulation. Also shown is the average absolute relative error in both diameter and pressure over the entire simulation period

This process is repeated for a chain of 10 lymphangions in a variety of pressure states. The final ten contractions of a 600 second simulation are averaged for all lymphangions to give an average of $\bar{Q}_{c}$ for the chain. This average for the differing pressure 
states can be observed in Figure 4.13. The relative error in this quantity due to removal of the inertial term is shown in Figure 4.9. As can be seen these errors are substantial with a near $20 \%$ overestimation and a $35 \%$ underestimation of the average flow rate in the worst case. The relationship between this error and the pressure state is not monotonic or clear. Little relationship appears to exist between the trans-mural pressure and the error. It appears that peak over estimations occur between pressure differences of -1 to $0 \mathrm{cmH}_{2} \mathrm{O}$. These results challenge the assumption that inertia plays no role in lymphatic pumping. However, the understanding of its role is far from complete.

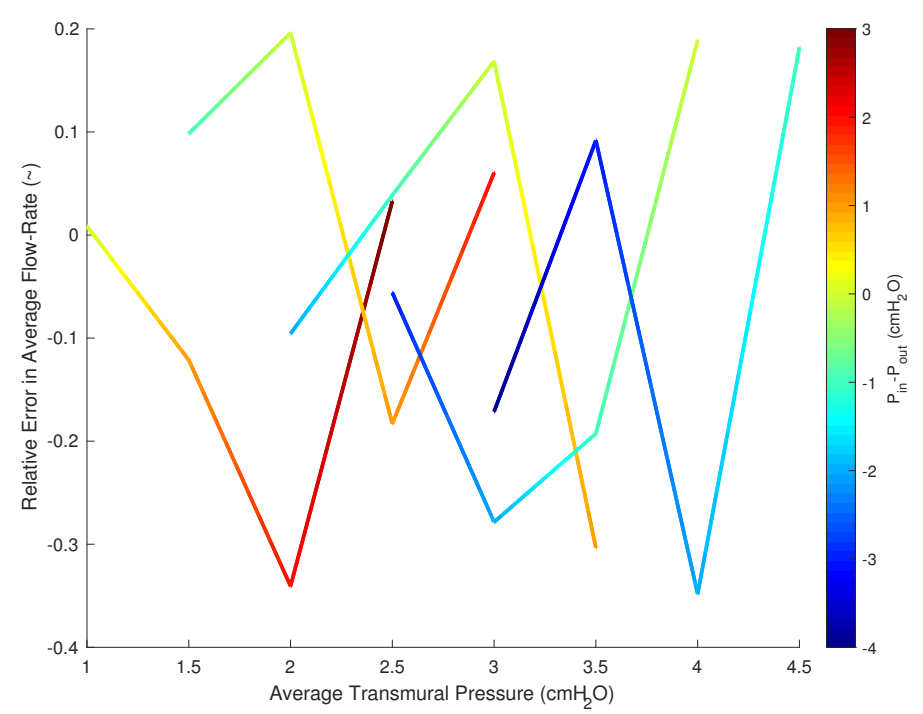

Figure 4.9: The relative error in the average flow rate, $\bar{Q}_{c}$, is shown for differing pressure conditions.

\subsubsection{Exploring the Effects of Pressure Loading}

The previously described mean $\bar{Q}_{c}$ is shown below in Figure 4.13. The relationship between chain pressure difference and the average flow rate is surprisingly consistent and linear, though a slight downward inflection can be noted at the higher chain pressures - a trend which is reversed at lower chain pressures. Higher trans-mural pressures seem to cause a widening in distribution of $\bar{Q}_{c}$ and steadily increase the ability of the network to pump. 


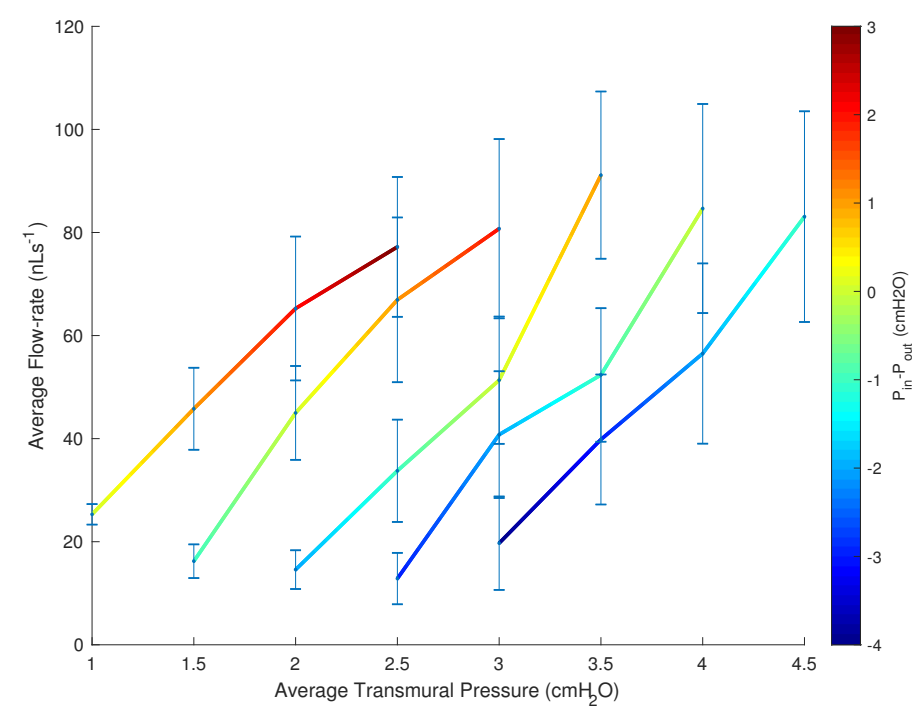

Figure 4.10: The effects of different pressure states on the average flow rate, mean $\bar{Q}_{c}$. Vertical bars indicate the standard distribution of $\bar{Q}_{c}$

This relationship is not held at the network scale. Figure 4.11 shows the distribution of $\bar{Q}_{c}$ at differing boundary pressure conditions, truncated at the 5th at 95th percentiles. As can be seen, trans-mural pressure conditions which would encourage flow increase the flow rate. However, flow-adverse pressure conditions do not appear to reduce the flow rate below some minimum. The distribution of flow rate grows more bimodal with increasingly adventitious pressure condition. All cases have an average trans-mural pressure of $4 \mathrm{cmH}_{2} \mathrm{O}$. However, higher trans-mural pressures associated increases the degree of retrograde flow. This property is responsible for a reduced efficiency at higher pressure. Figure 4.12 shows the distribution of $\eta_{c}$ or the network model at differing boundary pressure conditions, truncated between the 5th and 95th percentiles. A peak efficiency can be observed at a pressure difference of $3 \mathrm{cmH}_{2} \mathrm{O}$. 


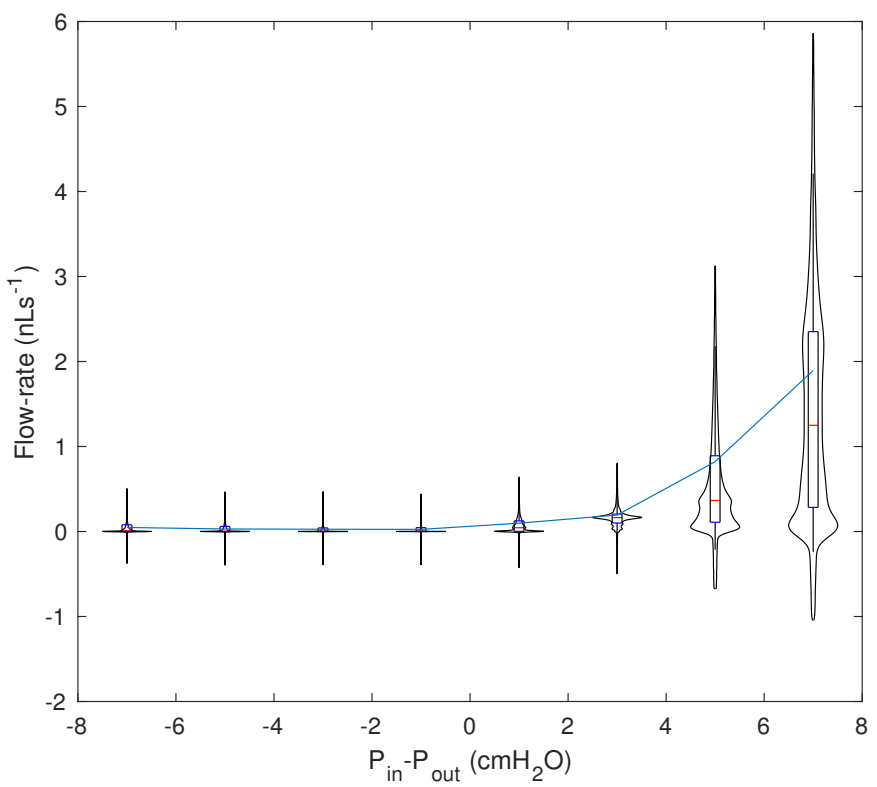

FIGURE 4.11: Violin plots of the distribution of $\bar{Q}_{c}$ is shown for the network model with differing pressure boundary conditions. The mean of $\bar{Q}_{c}$ is also shown in blue.

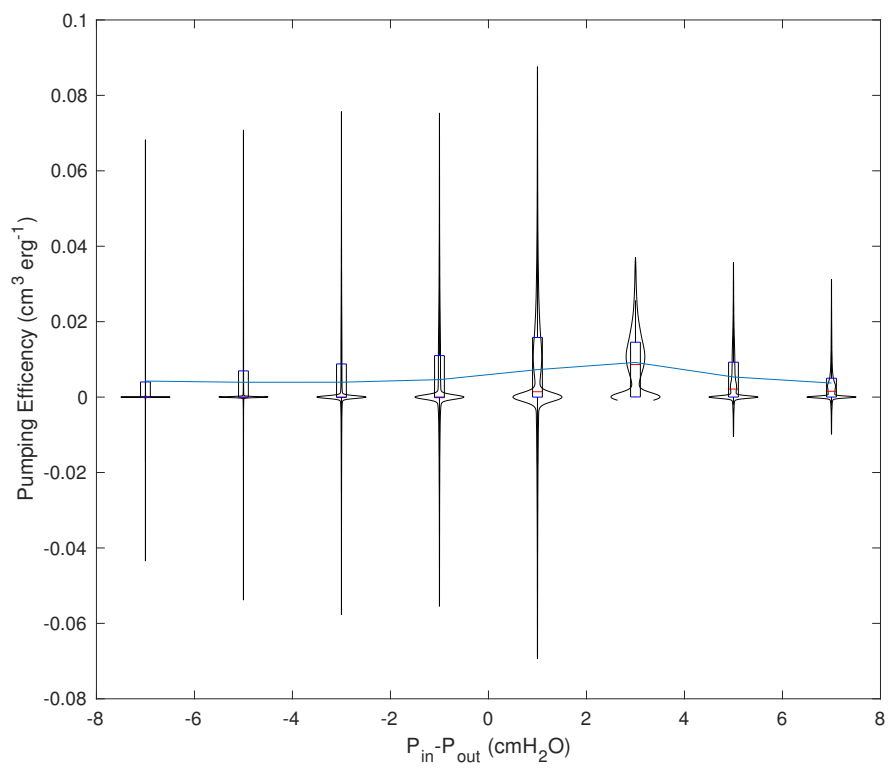

FIGURE 4.12: Violin plot of the distribution of the pumping efficiency, $\eta_{c}$, for differing pressure boundary conditions. 


\subsection{Discussion}

\subsubsection{Network Scale Model}

The network scale model as presented is insufficient in its transport of fluid in a manner that is likely not physiological. This insufficiency results in the accumulation of fluid in sections of the network as observed in Figure 4.7. This may be due to the parameters chosen, the network of lymphangion or the models absence of a regulatory mechanism. These will be discussed in turn and a hypothesis for the insufficiency at confluences proposed.

\subsubsection{Parameter Set}

The computational expense of the sensitivity analysis and parameter estimation precluded their use at the network scale. The consequence of this is that insight into the network model is limited. It is clear that phenomena observed at the single lymphangion and lymphangion chain scale are not replicated at the network scale. The sensitivity of parameters to pumping metrics at the network scale remains unknown. Similarly, while a manual fit to Davis data appears to at least be proximal to some minima the lack of a global consideration leaves open the possibility that a more appropriate minima in the parameter space exists, which may yield different results at the network scale.

\subsubsection{The Limb Network}

Sappey's networks and sketches have had clinical application. Sappey used the sketches to create a series of rules for deciding to which lymph node a section of skin drains, the sentinel node [60]. Starting in the 1950s with the invention of lymphangioscintigraphy physicians began to challenge Sappey's rules noting that the sentinel node could be ambiguous under some circumstances [70]. Authors have also challenged Sappey's observation that there was no pre-nodal communication between the deep and superficial 
lymphatics. It is essential to this work that this observation is correct. In a modern work, Suami et al. studied the upper-limb of cadavers using methods similar to Sappey which broadly supported Sappey's observations [60]. However, it is important to note that the sketches are being used in this work in a manner that Sappey could not have considered. It is likely that the diameters are illustrative. A better approach may be to select diameters such that the diameter of the parent and daughter vessels are decided to minimise the work required for transport, Murray's law is an example of such a system. This could minimise the insufficiency at the confluences.

\subsubsection{A Regulatory Mechanism}

A hypothesis for confluence insufficiency phenomenon is proposed. Under the current model lymphangions have a steady-state frequency of contraction dependent on the stretch of the vessel. At each confluence one of the parent vessels will have a steadystate frequency closer to the daughter vessel than the other parent vessels. This vessel is dominant over the other parents as its relative phase to the daughter vessel will change at the slowest rate maximising the time at which the relative pressure states of the parent and daughter vessel allow flow. The other parent vessels will accumulate fluid due to insufficiency, increasing their frequency with only a few contractions achieving meaningful flow. The downstream vessel would decrease its frequency as it depleted its fluid. However, the vessel is adequately supplied by the dominant parent and it does not adapt to the non-dominant parents. This behaviour would explain the observed insufficiency at confluences. In reality, lymphangions are also connected electrically and this communication may be how contractions are organised at confluences. No current lymphangion model of which the author is aware considers this electrical communication. 


\subsubsection{Discrepancies Between the Single Lymphangion Model and Pre- viously Published Experimental Data.}

In this section the model will be compared to previously published experimental data. In Figure 4.13, the model recapitulates the results found by Davis et al. [2]. Several phenomena are annotated and these will be used as a basis for a discussion the limitations of the model's agreement with experimental data.

\subsubsection{A: Pressure Curves}

The character of the pressure curves disagrees with the experimental results. However, in the results presented by Davis et al., the pressure was measured using a servo-null micropipette. This measures pressure by manipulating the pressure applied to a micropipette of hypertonic solution such that the electrical resistance is constant meaning there is now flow in or out of the micropipette. The frequency response of such a system is likely to be complex. Davis et al. raise the possibility that the frequency response of the micropipette may have been insufficient to accurately capture the lymphangion pressure peak [2]. Seki et al. examined the frequency response of similar system produced by the same manufacturer [5]. This frequency response was fitted with a Gaussian function, which was then used to form a finite impulse response filter. This filter was applied to the simulated data to emulate the behaviour of the servo-null micropipette. As can be seen while this does reduce the amplitude of the pressure curve it does not cause it to adopt the character observed experimentally. A widening of the pressure curve and a reduction in the amplitude could be achieved by increasing the eccentricity, such the contraction took place over a longer period. However, this would negatively effect the diameter fit and by extension the frequency of contraction. 

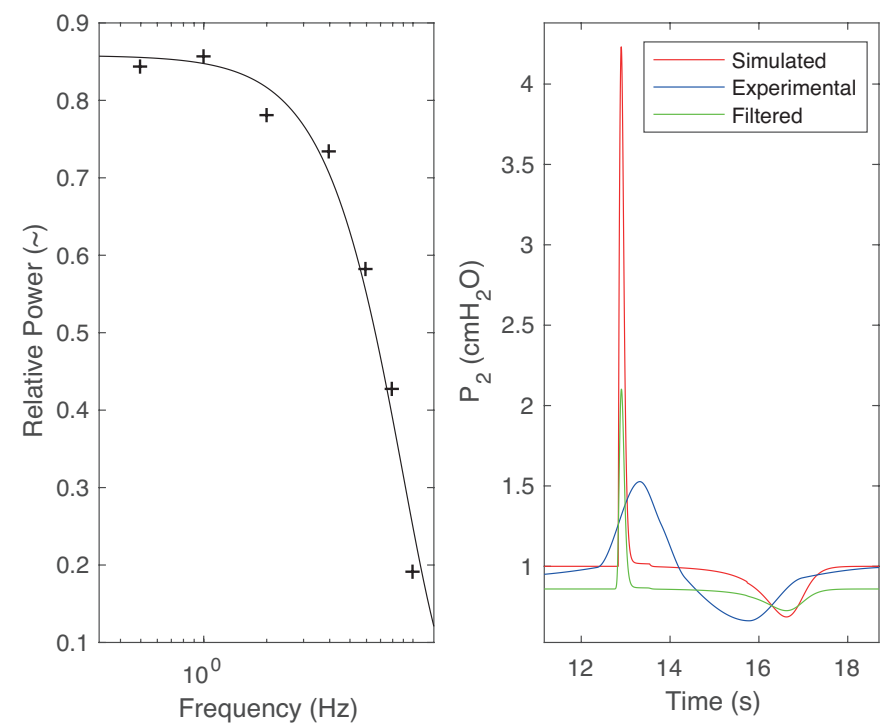

FiguRE 4.13: Left: the frequency response found by Seki et al. [5]. Right: Experimental data[2] is presented along with the fit shown from Figure 4.13 also shown is the filtered simulation result using a filter defined by the frequency response shown on the left.

\subsubsection{B: Insufficient Contraction}

In order to capture the increase in frequency associated with the increasing downstream pressure, the diameter at the end of contraction must increase as observed in the experimental data. The degree of contraction can be controlled through the $\alpha_{\mathrm{sf}}$. The behaviour referred to in section 4.4.2.6 means that in-order to characterise the frequency increase associated with an increasingly adverse pressure difference. $\alpha_{\mathrm{sf}}$ must be set such that the initial simulated contractions have a smaller reduction in diameter, than the initial contractions observed experimentally.

\subsubsection{C: Slope of Contraction}

As can be seen in Figure 4.13.C as well as all the sub-figures of Figure 3.4, the model overestimates the rate of contraction. There are two potential reasons for this; 1) a more complex length-dependent tension would capture this effect; 2) a force opposes 
contraction which is not considered by the model. In the first case, consider that the highly non-linear stiffness of the wall would require an equally non-linear contraction function to produce such a smooth, almost linear, contraction. The very low stiffness present at the end of contraction means the load of the muscle would have to be applied much more gradually than in any current published consideration in order to capture this effect. It is not clear what biological mechanism would account for this contractile behaviour. In the second case, there are two forces which could oppose the contraction. A force which resists the change in motion, an inertial force, or a force which resists the motion itself, a viscous force. The model already incorporates the viscous quality of the fluid domain which leaves only an inertial force or a viscosity in the wall. Viscoelastic wall properties have been previously been considered in 0D/1D arterial modelling [56]. It has also previously been incorporated into 1D lymphangion modelling [34]. Future work should seek to incorporate this behaviour into 0D modelling

\subsubsection{D: Relaxation Slope}

The slope of relaxation is also much sharper than observed experimentally, as can be seen in Figure 4.13.D. The argument made in section 4.4.2.3.C is not as convincing here as it occurs across a relatively linear stiffness regime and occurs over larger time-scales than the viscoelastic effects. Previous approaches have used more complex length-tension relationships in order to account for this phenomenon [41]. Future work could aim to build physiologically motivated models of lymphatic muscle cells. It is likely that a more physiologically motivated contraction model considering the length-tension relationship of the muscle fibre would allow greater insight into this phenomenon.

\subsubsection{E: Loss of Volume}

The experimental results appear to show a gradual loss of volume within the lymphangion as the pressure gradient grows more unfavourable. There would appear to be two 
mechanisms that would prevent this. Firstly, the diameter dependent frequency of contraction and secondly the Frank-Stirling principle - the general assumption made that the non-linear material properties regulate contractions such that output remains constant. Future work should seek to establish if this property is representative of lymphangions in general.

\subsubsection{F: Lack of Retrograde Fluid Motion}

The experimental data continues to show a diameter contraction when the downstream valve no longer opens due to retrograde motion through the upstream valve. This behaviour is not replicated by the model as the upstream valves do not open during this period. This suggests that the kinetics of the valve opening are not correct as the trans-valvular pressure generated due to the resistance of the valves during opening is sufficient to raise the pressure causing the upstream valves to close again. It may be that a fluid-structure interaction study of valve opening dynamics reveals the source of this behaviour.

\subsubsection{G,H: Refractory Period}

As can be seen in these sections of the experimental data there is a refractory period when the downstream pressure is relaxed. This refractory period is preceded and followed by large contractions. The first contraction after the refractory period is the largest contraction observed. As the first contraction after the relaxation of the downstream pressure condition would have the highest velocity this could be cause the wall-shear stress inhibitation phenomena that Contarino and Torro included but is absent from this work [35]. 

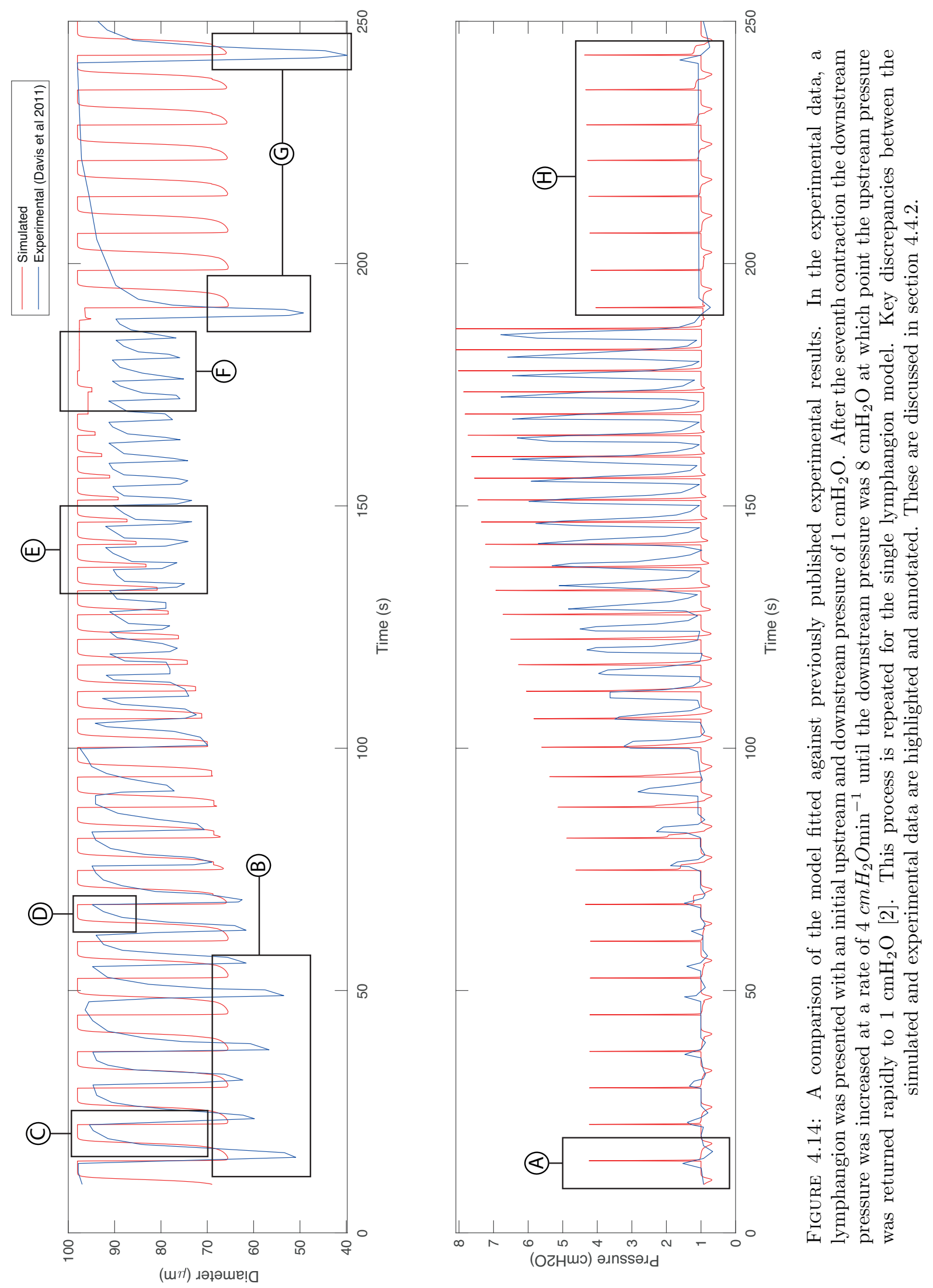


\subsection{Conclusion}

It is clear that much work is needed before large scale modelling of the lymphatic system can realise the potential benefits. The phenomenological approach taken in this work offered several benefits advantageous to its aim. The computational cost reduction of lumped modelling and the reduction of parameter sets through the use of experimentally derived relationships allowed the expansion in scale this work achieved. However, this approach does not allow the degree of insight offered by physiologically motivated modelling attempts undertaken by others [34][35]. Difficulties in achieving experimentally observed diameter and pressure responses are caused, in part, by the inflexibility of experimentally derived relationships. A physiologically motivated model of contraction capable of integrating calcium dynamics, nitric oxide dynamics and electrical coordination between lymphangions would be beneficial to future work. The sensitivity of these models to parameter variations add further difficulty to the problem of parameter estimation. The lymph nodes are represented in this work as a simple connection between lymphangions when in fact they are complex contractile structure that alter the composition of lymph and present variable resistances and compliances, little work has been done to couple lumped lymphangion models and lymph nodes.

The identification of a bimodal distribution of contraction periods as indicative of insufficiency may augment diagnosis. NIRF has been used previously to estimate the period of lymphatic contraction in human limbs [71]. Spectral analysis of NIRF signals could allow the identification of bimodal contraction period distributions as a metric for insufficiency. The reduced efficiency of lymphatic contractions at higher adventitious pressures could be used to inform the application of compressive garment use in lymphoedema patients.

The importance of inertia at the lymphangion scale can be debated, however as the scale of model approaches the organelle, organ or limb scale the consequences of the small changes in valve dynamics start to cause significant effects. It maybe possible to capture lymphatic phenomena, propose valid hypothesis and validate computational 
results without considering inertia, the assumption by the field that it is insignificant needs to be re-examined and future work in this field should examine the role inertia may play carefully, before proceeding without it. 



\section{Chapter 5}

\section{Breast Cancer Related}

\section{Lymphoedema and its Effect on Limb Shape.}

\subsection{Introduction}

Injury to the lymphatic system as a result of axial node dissection, radiation or sentinel node biopsy in the treatment and diagnosis of breast cancer is a common cause of secondary lymphoedema of the upper limb [72]. The removal of lymph nodes during an axial node dissection - and to a lesser extent a sentinel node biopsy - reduces the lymphatic pathways available to remove lymph from the interstitia. Radiation causes a general degradation of the exposed lymphatic vessels and nodes through mechanisms not currently entirely understood. Outside of the developed world the largest single cause of secondary lymphoedema is filariasis. However, within the US nearly all secondary lymphoedema patients develop the condition as a consequence of cancer or its treatment [73]. 


\subsubsection{The Diagnosis of Lymphoedema}

It is often stated that the "gold standard" of breast-cancer related lymphoedema (BCRL) diagnosis is by lymphangioscintigraphy (LAS). Its use is recommended by the International Union of Phlebology, the American Venous Forum and the International Society of Lymphology [74][75]. Under this method a contrast, usually albumin-bound technicium $99 \mathrm{~m}$, is injected intradermally or subcutaneously and the limb is imaged as the contrast is taken up by the lymphatic system. Analysis of the "pattern" produced allows qualitative statements about the lymphatic system to be made. For example no uptake of the contrast indicates no functioning lymphatics and is referred to as a "no-flow" pattern and is more associated with primary than secondary lymphoedema. A specific lymphatic injury can be found when the contrast is observed to stop progressing along a vessel and diffuse away from the vessel indicating it is undergoing reflux into the tissues around the injury. Several issues exist with this method: Firstly, it is important to note that the detection of an injury and the presence of oedema does not imply causation. Co-morbidities common to cancer patients mean that the oedema may have another origin i.e. renal failure. Secondly, no quantitive measure of the insufficiency is available. Thirdly, there is no accepted standard for this procedure [74]. Finally, the author is aware of no study in which patients undergoing breast cancer surgery were routinely subjected to LAS - though the expense and risks associated with nuclear imaging may explain this: This makes assessing the incidence of BCRL through LAS difficult. The rise of near infra-red imaging (NIRF) with idocyanine green (ICG) reduces the invasive nature of the procedure which may lead to its wide spread use [76][62][77]. It has been routinely used in one study which will be discussed later [78].

Outside of LAS, diagnostic methods for lymphoedema involve assessing a history indicative of lymphatic injury, a "lymphoedema" phenotype of the swelling and an assessment of the degree of oedema. This assessment of the degree of oedema appears to be responsible for the wide array of incidence estimates.

Water displacement, opto-electrical volumetry, and tape measurement are the 
three methods typically used to assess the degree of oedema. Water displacement measures the volume of the limb by Archimedes' principle; the limb is submerged in water and the volume calculated by measuring the water displaced. This method has been referred to as the gold standard for limb volume measurement [79]. However, in practice it has been described as below.

"In practice when dealing with the limbs, this procedure requires a whole collection of different containers and apparatus that renders it unpractical, messy and time consuming. Furthermore with regards to hygiene, since people with lymphoedema often have concomitant skin alterations, the appropriateness of this procedure is questionable and it is also not suitable for patients in the immediate postoperative period." - Belgrado et al. [80].

Opto-electrical volumetry measures the semi-major and minor axis of the limb using a device called a perometer. The perometer uses two perpendicular arrays of infra-red LEDs to cast shows onto two perpendicular sensors. The width of the shadows cast on the two sensors are measured as the arrays are moved along the length of the arm. The perometer assumes the arm is an ellipse with a semi-major and minor axis same as the lengths of the two shadows. A diagram demonstrating the operation of a perometer is shown in Figure 5.1. The final and most commonly used method to determine the volume of the limb is by tape measurement. A non-elastic tape is used to measure the circumference at multiple locations along the length of the limb. These are then used to calculate the volume assuming a series of circularly cross-sectioned frustra. 


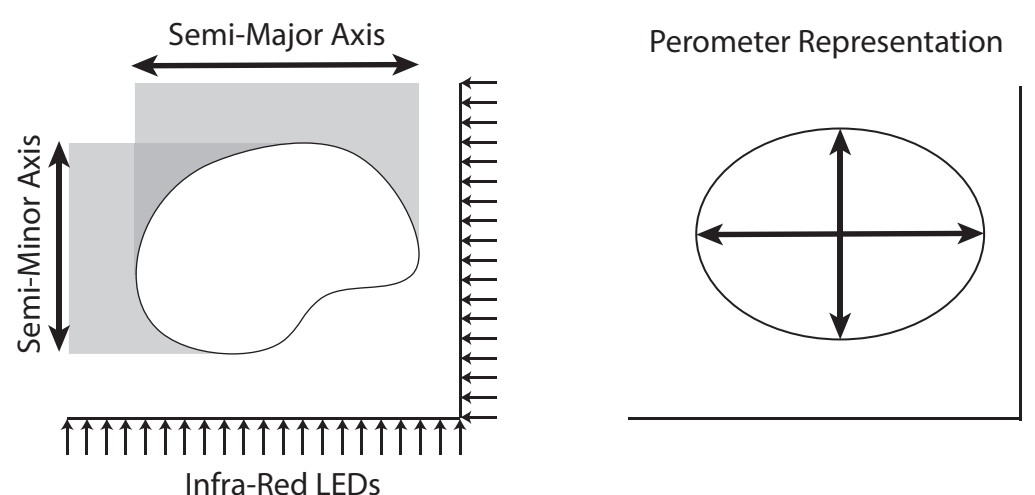

Figure 5.1: A diagram explaining the operating principle of a perometer. A frame containing two perpendicular arrays of infra-red LEDs opposed by sensors are moved with both arrays orthogonal to the arm. The cross-section is assumed to be a ellipse, with the semi-major and minor axis having the same lengths as the shadows cast by the limb.

Cheville et al. examined differing strategies for grading lymphoedema and their consequences on the reported incidence of lymphoedema [7]. Figure 5.1 expands on their analysis by combining seven of the nine papers they considered with three more modern studies and refining the focus to axillary node dissections (ALND) - a common cause of BRCL. Two studies were exempted in the first instance because the study used a management grading of lymphoedema which precludes quantitative diagnosis and in the second instance because no ALND patients were considered. In one study a small percentage of sentinel node biopsies (SNB) - 6\% - are included because the results were not presented in a way that permitted their removal. As SNB patients are less likely to be diagnosed with lymphoedema it is assumed this will yield a slight underestimation of the incidence rate.

A large range of differing estimates of the incidence rate are found for similar methods. The three highest circumference discrepancy incidences Pavia [78], Petrek [81] and Gerber [82] each measure the circumference in a relatively large number of places along the limbs length; 7, 4 and 3 places respectively. This implies that the position and or quantity of measurements is important. This hypothesis is further compounded given the high incidences found by perometer and water displacement methods, Stout Gergich [83] and Sergerstrom [84], which measure continuously along the length of the 
limb. The absence of a properly controlled study on the methods of diagnosis for lymphoedema means the effects of different methods of diagnosis cannot be determined. However, without a gold standard against which to assess this methods of diagnosis; it is impossible to deconvolve the "true" incidence of lymphoedema. The Akita study [78] used ICG NIRF to offer a direct measure of lymphatic function, which could serve this purpose. The Akita study [78] has the highest reported incidence of any none selfreporting study, which could imply that lymphoedema was under-represented in the other clinical trials [77]. However, that statement is complicated as some patients in that study were administered axilla radiation therapy, which is associated with lymphoedema. It remains clear that no standard method for assessing lymphoedema exists in clinical trials, the methods used are varied and lack an evidential basis and the highest incidence is in patient self-reporting. The elevated findings of ICG NIRF are particularly significant because they fundamentally do not gauge the degree of lymphoedema but instead identify the lymphodema phenotype. It is this observation that forms the following assumption. Inconsistencies in lymphoedema incidence are due, in part, to an over-reliance on identifying the degree of oedema instead of the character of oedema. 


\begin{tabular}{|c|c|c|c|c|}
\hline Study & $\mathrm{n}$ & Procedure & Diagnostic Criteria & $\begin{array}{l}\text { Reported } \\
\text { Incidence }\end{array}$ \\
\hline \multirow[t]{3}{*}{ Paiva $2016[78]$} & 250 & ALND & Inter-limb circumference discrepancy & \\
\hline & & or SNB $(6 \%)$ & & \\
\hline & & & $>2 \mathrm{~cm}$ (any of seven) & $44.5 \%$ \\
\hline \multirow{3}{*}{ Akita 2016 [78] } & $71 \ddagger$ & ALND & ICG NIRF & \\
\hline & & \& Axilla Radiation & & \\
\hline & & & Diffuse or Stardust & $45.8 \%$ \\
\hline \multirow[t]{8}{*}{ Stout Gergich 2009 [83] } & $71 \ddagger$ & ALND & Inter-limb Perometer volume discrepancy & \\
\hline & & & $>2 \%$ & $45 \%$ \\
\hline & 923 & ALND & Inter-limb circumference discrepancy & \\
\hline & & \& Mastectomy & + Self Reported feeling of oedema & \\
\hline & & & Mild $<0.5$ in (any of 4$)$ & $19 \%$ \\
\hline & & & Moderate $0.5-2.0$ in (any of 4 ) & $17 \%$ \\
\hline & & & Severe $>2.0$ in $($ any of 4$)$ & $13 \%$ \\
\hline & & & & Total $=49 \%$ \\
\hline \multirow[t]{6}{*}{ Schrenk $1999[85] \dagger$} & 35 & ALND & Self report & \\
\hline & & \& Mastectomy $(26 \%)$ & swelling \& function & \\
\hline & & & Mild & $40 \%$ \\
\hline & & & Moderate & $14 \%$ \\
\hline & & & Severe & $0 \%$ \\
\hline & & & & Total $=54 \%$ \\
\hline \multirow[t]{3}{*}{ Liljegen $1997[86] \dagger$} & 35 & ALND & Tape measure & \\
\hline & & & volume difference & \\
\hline & & & $>100 \mathrm{ml}$ & $11.2 \%$ \\
\hline \multirow[t]{2}{*}{ Kiel $1996[87] \dagger$} & 183 & ALND & Inter-limb circumference discrepancy & \\
\hline & & + Axilla radiation $(2.2 \%)$ & $>1 \mathrm{~cm}$ (any of two) & $35 \%$ \\
\hline \multirow[t]{2}{*}{ Keramopoulos $1993[88] \dagger$} & $72 \ddagger$ & ALND & Inter-limb circumference discrepancy & \\
\hline & & Masectomy & $>2 \mathrm{~cm}$ (any of two) & $17 \%$ \\
\hline \multirow[t]{2}{*}{ Gerber $1992[82] \dagger$} & $61 \ddagger$ & ALND & Inter-limb circumference discrepancy & \\
\hline & & & $>2 \mathrm{~cm}$ (any of three) & $47 \%$ \\
\hline \multirow[t]{2}{*}{ Sergerstrom $1991[84] \dagger$} & 93 & ALND & Water Displacement & \\
\hline & & + Axilla radiation $(61 \%)$ & $>150 \mathrm{ml}$ & $43 \%$ \\
\hline \multirow[t]{2}{*}{ Werner $1991[89] \dagger$} & 282 & ALND & Inter-limb circumference discrepancy & \\
\hline & & +Axilla radiation $(23 \%)$ & $>2.5 \mathrm{~cm}$ (any of two) & $19.5 \%$ \\
\hline \multirow{2}{*}{ Lin $1993[90] \dagger$} & 122 & ALND & Inter-limb circumference discrepancy & \\
\hline & & & $>2 \mathrm{~cm}$ (any of two) & $24 \%$ \\
\hline
\end{tabular}

TABLE 5.1: A table of diagnostic criteria used in clinical studies of BCRL and their reported incidence. $\dagger$ from Cheville et al.[7]. $\ddagger$ Only ALND patients are produced from a study which included non-ALND patients. Non-axilla targeted radiation is not considered though some patients received it. Chemotherapy was given to most but not all patients. Modified radical mastectomies are omitted, stated mastectomies are simple or segmented

A variety of classifications for grading lymphoedema exist; of these, we will consider the International society of Lymphology grading(ISL), the American Physical Therapy Association lymphoedema Criteria(APTA), the latent effect of normal tissues (LENT/SOMA) measure and the common toxicity criteria (CTCv3).

The ISL has four stages: Stage 0 or sub-clinical, where swelling is not seen; 
stage I, where swelling is and can be transient and is alleviated by elevation; stage II where swelling is constant and pitting; stage III where the tissue is hard and fibrotic. There are also four severities based on volume increase. sub-clinical 3-5\%, mild 5\%-20\%, moderate $20-40 \%$ and severe $>40 \%$. The ISL consensus document also notes the use of minimal for a $5-10 \%$ difference[75].

The APTA has three grades of severity defined based on a the maximum inter-limb circumference discrepancy; mild, $<3 \mathrm{~cm}$; moderate, $3-5 \mathrm{~cm}$ and severe, $>5 \mathrm{~cm}$. LENT/SOMA has four grades based on the same metric; grade I, 2-4cm; grade II, 4-6cm; grade III, $>6 \mathrm{~cm}$ and grade IV is an arm with no function. In the cases of upper-limb CTCv3 [7] grades lymphoedema as follows:

\begin{tabular}{l|l} 
Grade & Characterisation \\
\hline 1 & $\begin{array}{l}5 \%-10 \% \text { inter-limb discrepancy in volume or circumference } \\
\text { at point of greatest visible difference; swelling or obscuration } \\
\text { of anatomic architecture on close inspection; pitting oedema }\end{array}$ \\
\hline 2 & $\begin{array}{l}\text { 10-30\% inter-limb discrepancy in volume or circumference at } \\
\text { point of greatest visible difference; readily apparent obscu- } \\
\text { ration of anatomic architecture; obliteration of skin folds; } \\
\text { readily apparent deviation from normal anatomic contour }\end{array}$ \\
\hline 3 & $\begin{array}{l}30 \% \text { inter-limb discrepancy in volume; lymphorrhea; gross } \\
\text { deviation from normal anatomic contour; interfering with } \\
\text { ADL }\end{array}$ \\
\hline 4 & $\begin{array}{l}\text { Progression to malignancy (i.e., lymphangiosarcoma); am- } \\
\text { putation indicated; disabling }\end{array}$
\end{tabular}

TABLE 5.2: A table showing the CTCv3 grading for lymphoedema as defined in [7]

Present in the CTCv3 but not included in CTCv2 or the other grading systems is the presence of shape as a metric of lymphoedema. It is important to note that all three clinical definitions in the CTCv3 involve shape with the mildest case being noticeable on "close inspection" and the standard rising to "readily apparent" and finally to "gross 
deviation". The reversal of shape changes during the development of lymphoedema has been considered an aim of treatment [63].

\subsubsection{Consequences of an Earlier Diagnosis}

In order to motivate a method of diagnostic metric better able to quantify early lymphoedema the potential benefits must be discussed. The relationship between patient outcomes and early diagnosis is not straightforward. It has been recommended that early assessment of lymphoedema should not be used as an indicator of prognosis, given the rate of spontaneous improvement [84]. However, the effectiveness of lymphoedema management is significantly improved if applied when the oedematous volume is still small [91]. Lymphoedema leads to a odematous volume that without treatment steadily increases in time [92]. As Lymphatic function is compromised before odematous volume becomes detectable [78]: it follows that early diagnosis is unreliable because it is inaccurate but early treatment is beneficial. Diagnosis by the odematous volume is complicated by the mean difference in volume of $2.2 \%$ found between dominant and non-dominant arms [93], the presence of peri-operational swelling and the fact that early dysfunction of the lymphatic system causes undetectable odematous volume. An alternative method to calculate the relative fluid content of the tissues has been well described in the form of multi-frequency bio-impedance analysis and this method has been shown to be able to diagnose lymphoedema [94][95]. However, it is still a method of volume measurement and requires comparison to the unaffected limb of that same patient.

\subsection{Developing a New Diagnostic Method}

A new method of assessing lymphoedema has been proposed. 3D stereo optical cameras can measure produce distance images. Images taken at multiple angles around a limb can be used to reconstruct the limb surface [96]. These scans don't suffer from the practical issues associated with water displacement and offer more geometric information than 
opto-electrical volumetry. This chapter attempts to see if this geometric data can be exploited to improve early diagnosis. The author takes the following assumption. A subclinical form of lymphoedema exists, which if accurately diagnosed would be beneficial to patient outcomes. These early changes are first noted by the patient and could be responsible for the higher incidence recorded by self-reporting in the early detection [97]. However, they need to be separated from post-surgical effects which are responsible for the apparent spontaneous improvement. Volumetric measurement is insufficient to assess the small increase in volume and the changes in shape too small to be noticeable on "close inspection". Whilst some of these assumptions are supported in literature, a thorough establishment of them is beyond the scope of this work. However, they motivate the following hypothesis. The "character" of swelling can be quantified as a change in shape and this metric will significantly differ between mildly lymphoedematous and non-lymphoedematous limbs. The work in this Chapter sets out to define a metric of shape and test its suitability as an indicator of lymphoedema.

\subsection{Methods}

The following study modality was adopted to allow an exploration of metrics for lymphoedema while preventing over-fitting by preserving the integrity of limited patient data. 3D limb scans for 24 female patients (48 limbs) aged between 29 and 76, with mild unilateral lymphoedema, were scanned by Kevork Karakashian (Swansea University) as part of a Lymphaticovenular anastomosis (LVA) service review [96]. Three patients (6 limbs) were selected at random and removed from the study. These unaffected limbs would have a lymphoedema model induced in-silico through finite element analysis (FEA) and the resulting geometries subjected to manifold harmonic analysis (MHA) in order to examine changes in shape. The affected arms then have increasing degrees of multiplicative and additive noise added to check the robustness of the metric to noise. After these steps a single metric was developed which was then tested against the 21 remaining patients to assess its validity. An overview of the study design can be seen below in Figure 5.2. 


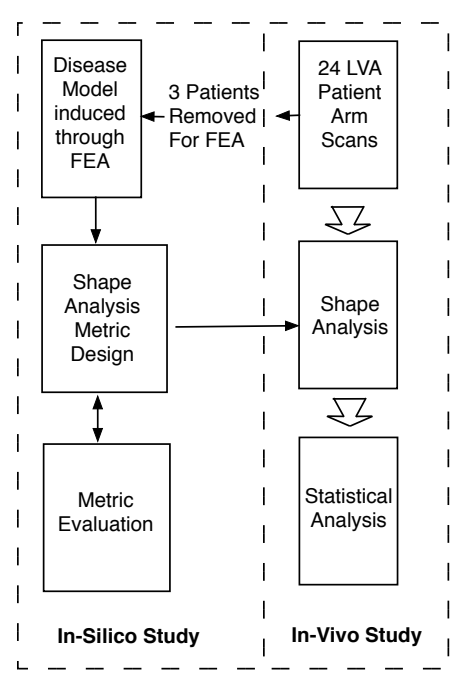

Figure 5.2: A flowchart showing the study design considering upper limb scans for group of 24 lymphoedema patients. In an in-silico study, three patients of this group were used to develop a shape-based metric that was then used in a statistical shape analysis of the remaining 21 patients.

\subsubsection{Manifold Harmonic Analysis}

Manifold harmonic analysis allows the quantification of shape and has previously been used to perform shape analysis, editing, and look-up [98]. It is suited to patient imaging use due to its robustness to changes in local topology, which is variable in the unstructured type meshes associated with patient-specific meshing [99]. However, its greatest benefit lies in the fact that the MHT is invariant to changes in pose [98]. This means that MHT derived metrics of shape will be independent of the position the arm is help during imaging. Manifold harmonic analysis has previously been used in an analysis of the shape of carpal bones [100] but has not previously been used to develop patient specific metrics [100]. The manifold harmonic transform (MHT) allows an analogue of the Fourier series for triangulated manifolds. MHA is performed by eigendecomposition of a discrete Laplacian yielding a series of eigenvalues which represent frequency and eigenvectors which represent the contribution each node makes to that frequency. Briefly, MHA allows Fourier like transformations to be performed on manifolds using the eigenvectors of the Laplace-Beltrami operator [99]. In standard Fourier analysis the 
function, $f(x)$, is decomposed into a trigonometric series as,

$$
f(x)=\sum_{n=-\infty}^{\infty} \hat{f}(n) e^{i \omega_{n} x}
$$

where $\omega=2 \pi n / T$, with $T$ the period and $\hat{f}(n)$ is the Fourier transform, whilst $f(x)$ is decomposed onto an orthonormal basis, $e^{i \omega_{n} x}$. Each Fourier basis function satisfies the Helmholtz equation of the real line.

$$
-\frac{\partial^{2} \phi_{k}(x)}{\partial x^{2}}=\omega_{k}^{2} \phi_{k}(x)
$$

Thus the $k$-th Fourier basis function is the $k$-th eigenvector of the 1-D Laplace operator. It is this property that is used to generalise the Fourier transform to higher dimensions. Levy[99] sought a family of functions $\left\{\phi_{k}(x)\right\}$ that could form a complete basis for arbitrary manifolds, such that any square-integrable function defined on a manifold, M, can be decomposed into a linear combination of this basis, $\left\{\phi_{k}(x)\right\}$. In 1-D Euclidean space $\left\{\phi_{k}(x)\right\}$ are the eigenvectors of the Laplace operator.

\subsubsection{The Laplace-Beltrami Operator}

The Laplace operator can be generalised on a manifold as the Laplace-Beltrami operator, $\Delta_{\mathcal{M}}$. Many attempts to construct a discrete Laplace-Beltrami operator have been made. Wardetzky[101] notes it is impossible to construct a discrete Laplacian that converges to the smooth Laplacian and is also symmetric for general meshes. However, a popular scheme is proposed by Meyer et al. [6]. Consider any triangulation with a vertices list, $\mathbf{V}$ of size $\left(n_{v}, 3\right)$, and an associated edge list $\mathbf{E}$. Now a geometric weight matrix $\mathbf{W}$ of size $\left(n_{v}, n_{v}\right)$ can be defined as follows.

$$
w_{i j}= \begin{cases}-\frac{\cot \beta_{i j}+\cot \dot{\beta}_{i j}}{2} & \text { if }(i, j) \in \mathbf{E} \\ \sum_{k} \frac{\cot \beta_{i k}+\cot \dot{\beta}_{i k}}{2},(i, k) \in \mathbf{E} & \text { if } i=j \\ 0 & \text { else }\end{cases}
$$


Where $\beta_{i j}$ and $\dot{\beta}_{i j}$ are the measures of the angles opposing the edge $(i, j)$. A diagonal matrix, $\mathbf{A}$, can be defined where $\mathbf{A}_{i i}$ equals the finite Voronoi area of the $i^{\text {th }}$ member of $\mathbf{V}$, calculated using the method described by Meyer et al. [6]. An overview of the construction of the weights matrices is described in Figure 5.3.

The generalised eigenvector problem $\mathbf{W} \boldsymbol{\Phi}=\boldsymbol{\Lambda} \mathbf{\Phi} \mathbf{A}[98]$ can be solved to yield a $n_{v}$-by- $n_{v}$ matrix of eigenvectors $\boldsymbol{\Phi}$, and the eigenvalue matrix, $\boldsymbol{\Lambda}$, whose diagonal, corresponds to the square of the fundamental frequencies those bases represent. We can therefore transform $\mathbf{V}$ to give $\mathbf{V}=\mathbf{V}^{T} A \boldsymbol{\Phi}$ where $\mathbf{V}$ is the spectral representation. Upon the boundaries only one angle opposing the edge is found. Hence, $\cot \left(\dot{\beta}_{i j}\right)$ does not exist, and only the finite Voronoi region is considered [99].

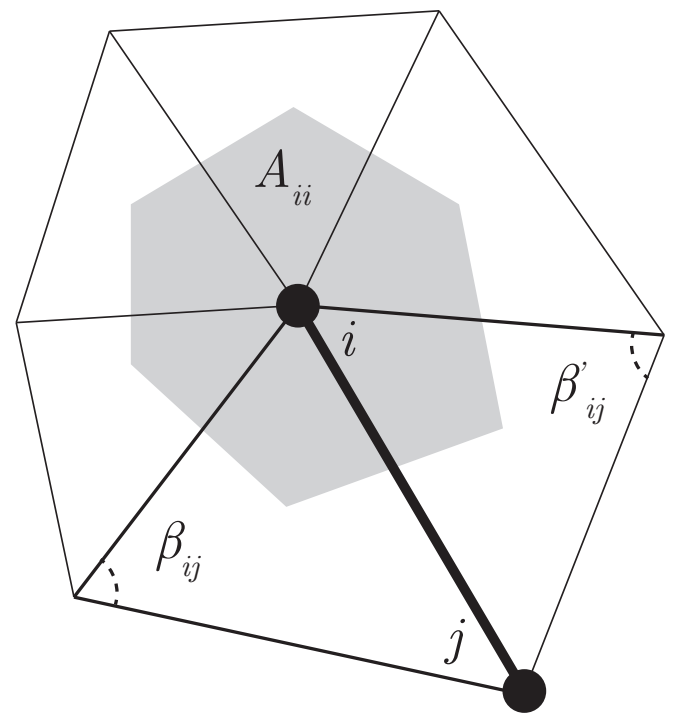

Figure 5.3: A figure demonstrating the Meyer et al. construction of the LaplaceBeltrami operator [6]. For a node, $i$, the area weight, $A_{i i}$, is the Voronoi area associated with the node. For each associated edge $(i, j)$ the weight $w_{i j}$ is found as a function of the two corners that oppose $i j, \beta_{i j}$. For the leading diagonal of $\mathbf{W}$ the values are the negation of the sum of every weight associated with that node.

\subsubsection{Global Point Signature}

Shape analysis performed using the Laplace-Beltrami spectra directly has been proposed [102][98]. However, the techniques have been improved by the introduction of 
global point signature. The global point signature (GPS) normalises the eigendecomposition of the Laplace-Beltrami operator by the frequency to yield a multidimensional spectral embedding where the distances between vertices in that embedding are more meaningful, while still conserving the original properties [103]. The embedding of the geometry is constructed from the eigendecomposition of the Laplace-Beltrami operator. Each column of $\boldsymbol{\Phi}$ is an eigenvector which represents a sinusoidal function on the surface of the geometry and each element of that eigenvector represents the contribution of the associated vertex to that mode. A limb geometry coloured by the normalised eigenvectors for some low frequency modes is shown below in Figure 5.4. Similarly each row of

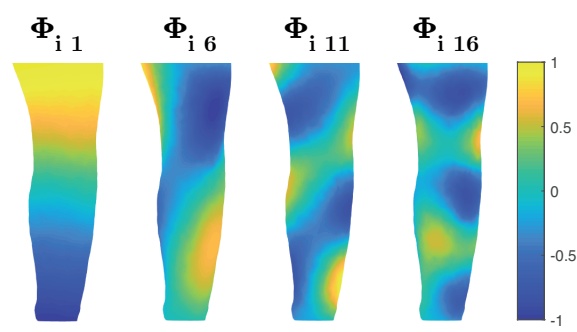

FiguRE 5.4: The first, sixth, eleventh and sixteenth eigenvectors of the LaplaceBeltrami operator are shown for the case one limb geometry. Colour is normalised eigenvector coffecient.

$\Phi$ represents a multidimensional spectral embedding of each vertex with each dimension representing that points contribution to each sinusoidal function. This embedding is called the global point signature (GPS), the $i$ th vertex of $\mathrm{V}$ would be embedded for $n$ eigenvectors as follows.

$$
\hat{\phi(n)_{i}}=\left[\frac{\phi_{1 i}}{\lambda_{1}}, \frac{\phi_{2 i}}{\lambda_{2}} \ldots \frac{\phi_{n i}}{\lambda_{n}}\right]
$$

Reducing $n$ truncates the series and is equivalent to a low-pass filter. 


\subsubsection{G2-distribution Metrics}

The G2-distribution is the distribution of pairwise distances between each point of the geometries GPS embedding [103].

$$
\mathbf{D}_{i j}(n)(\boldsymbol{\Phi})=\sum_{k=1}^{n} \sqrt{\hat{\phi}_{i k}-\hat{\phi}_{j k}}
$$

It is important to note that the zero-th eigenvector and eigenvalue, $\phi_{0}=[0, . ., 0]$ and $\lambda_{0}=0$ is not aggregated into the GPS as the resultant GPS would have an undefined value. Rustamov observed the significance of distances within this space and their relation to Green's functions [103]. Briefly, the inner product of two points within the GPS domain is equal to the Green's function of the two points. Consider an elastic surface, $S(\mathbf{x})$, subject to some load, $f(\mathbf{x})$, such that it undergoes displacement, $\mathbf{u}(\mathbf{x})$.

$$
\nabla^{2} \mathbf{u}=f(\mathbf{x})
$$

Now suppose that the load was concentrated on a point, q, i.e. $f(\mathbf{x})=\delta(\mathbf{x}-\mathbf{q})$. One way to solve this equation is by convolving the load with a Green's function $G(\mathbf{x}, \mathbf{q})[104]$.

$$
\mathbf{u}(\mathbf{x})=\int_{S} f(\mathbf{q}) G(\mathbf{x}, \mathbf{q}) \mathrm{d} \mathbf{q}
$$

As can be seen for any point, $\mathbf{p} \neq \mathbf{q}$ the function $G(\mathbf{p}, \mathbf{q})$ represents the consequence of loading point $\mathbf{q}$ on the displacement at point $\mathbf{p}$. As Rustamov notes the magnitude of Green's function represents the 'togetherness' of two points and it is this quality that gives meaningful representation to distances within the GPS embedding [103]. These distances can be represented as a distribution drawn from the GPS truncated to $n=m_{1}$ $\mathbf{d}\left(m_{1}\right)=\left\{\mathbf{D}_{i j}\left(m_{1}\right)(\mathbf{\Phi}): i \neq j\right\}$ [105]. Where $m_{1}$ is a truncation point which will be found later. It is by studying this distribution that shape analysis can be performed. The similarity of shapes can be found by comparing metrics constructed from the distribution of these distances [105]. Probability density functions can be constructed for this distribution using a histogram method. When selecting the number of bins, two factors need to be considered. Firstly, a large enough number of bins to ensure sensitivity to 
changes between distributions and secondly, a small enough number of bins to reveal the underlying distribution. By inspection, a histogram of 1000 bins of equal width was placed across the range of G2 values for each arm and the resultant count normalised by the total number of counts to give each bin a probability of occupancy.

\subsubsection{Entropy and Redundancy}

There is a conceptual relationship between informational entropy and lymphoedema, the swelling can be considered as the progression of the cutaneous tissues toward a minimal surface, obscuring the detail of muscle and bone that lies beneath. The Shannon entropy of the G2-distribution, $H\left(\mathbf{d}\left(m_{1}\right)\right)$, gives a metric of this process, its value falling as the G2-distances become more consistent representing a homogenisation of the geometry. Through the histogram method, a discrete estimation of the probability distribution, $P\left(\mathbf{d}\left(m_{1}\right)\right)$, can be constructed and the Shannon entropy of the distribution can be calculated as follows with $i$ representing the $i$ th bin of the histogram.

$$
H\left(m_{1}\right)=-\sum_{i} P\left(\mathbf{d}\left(m_{1}\right)_{i}\right) \log P\left(\mathbf{d}\left(m_{1}\right)_{i}\right)
$$

However, the Shannon entropy alone requires a context for interpretation. It can be normalised as a ratio of the maximum entropy possible in the context. This expression, called redundancy, is a measure of the informational content as compared to the maximum possible within the context [106]. In order to find the maximum entropy, consider the Fiedler vector, $\phi_{i} 1$, shown on a limb surface in the left hand plot of Figure 5.4. This function has one turning point over the surface, each higher frequency eigenvector will turn more than once. It is this property that causes the pairwise distances at this frequency-scale to represent the least-ordered function with the highest entropy, $H(d(1))$. Thus the entropy of pairwise distances constructed from $\hat{\phi}_{i} 1$ represent a maximum entropy for the GPS embedding.

$$
R=1-\frac{H\left(\mathbf{d}\left(m_{1}\right)\right)}{H(\mathbf{d}(1))}
$$


A redundancy, $R$, can be formulated as above to represent the additional order added by the aggregation of higher frequencies in the GPS embedding which lower the entropy when compared to the Fiedler vector. The geometry can be considered as the superposition of these ordered low-entropy, underlying features and a high-entropy cutaneous tissue which covers them. Measuring the redundancy of information, $R$, in the entropy of the G2-distribution as compared to the entropy of a G2-distribution defined from the Fiedler vector, allows a measure of the order or structure of the signature, which is not present in the limb's most basic description.

\subsubsection{Noise}

In manifold harmonic analysis, it is not common to calculate all the possible eigenvectors for the given mesh; both to reduce computational expense and exclude noise which often dominates for the higher frequency eigenvectors. Other work in the field of manifold harmonic analysis has found that the number of eigenvectors required to recapitulate a geometry sufficient for most shape-analysis objectives is a relatively small subset of the total, i.e. around 1\% [99][98]. Given the role of eigenvectors of the Laplace-Beltrami operator in manifold harmonic analysis and the Fourier-analogous nature of the manifold harmonic transform, a method for assessing the first noise dominant eigenvector is proposed. The power-frequency spectrum for a manifold can be found from the manifold harmonic transform in a similar manner to that seen in Fourier analysis. For each eigenvalue, $\lambda_{i}$, an associated power, $P_{i}$, can be found by taking a Euclidean norm of the $i$ th row of $\mathbf{V}$. The power-frequency spectra for each of the three limbs in the FEA study is shown below in Figure 5.5. Based on the principle that the random nature of noise means there should be no correlation between the power of noise and its frequency. Thus the first mode where noise is dominant can be identified as the largest eigenvalue for which the correlation between power and frequency can be established. To achieve this the piecewise-linear function, $\hat{P}(m)$, is fitted in a least-squares manner to identify 
the first eigenvalue at which noise is dominant, $m_{1}$,

$$
\log (\hat{P}(m))=\log \left(\bar{P}_{n}\right)+\left\{\begin{array}{l}
0 m \leq m_{1}, \\
k m \text { else }
\end{array}\right.
$$

with $\bar{P}_{n}$ representing the mean logarithm of noise-power and $k$ a coefficient of linearregression of the relationship between power and frequency. For the three limbs under consideration in the in-silico study $m_{1}$ was found to be $40.67 \pm 2.33$. In order to ensure a consistent number of modes in further study, a more conservative value of $m_{1}=50$ was used.

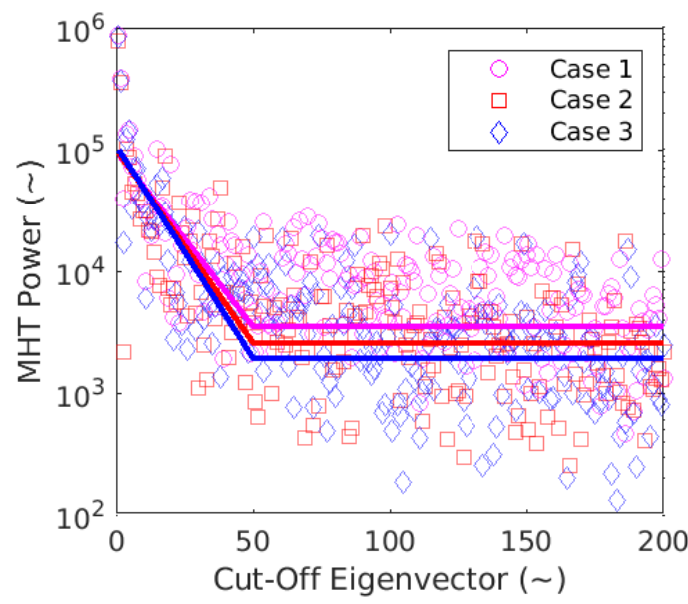

FIGURE 5.5: A plot showing the power-eigenvalue number relationship for the three in-silico geometries. The solid lines represent least-squares fits of a 'dog-leg' function with the inflection point representing the point of noise dominance. The eigenvalue numbers are such that the Fiedler vector would have an eigenvalue number of 1 and the highest frequency would be numbered $n$.

\subsubsection{Finite Element Analysis}

Finite element analysis could be used to develop models of lymphoedema that had direct diagnostic applicability. However, this would require models which considered the complex phenomena that occur. Examples of these include, the varying compliance of the tissues, strain dependent permeability and the remodelling of the skin to reduce stress over time. Even if such a model was constructed it would then require extensive 
simulation to build a understanding of how different fluid-loading conditions effected limb shape. These changes in limb shape would then need to be quantified. It is also possible that such a modality could be used in a patient-specific manner. With individual patient geometries being modelled to aid in patient prognosis. However, in this work a much simpler approach is taken. For the three limbs that have been set aside from the main study. A crude and simple model of lymphoedema is imposed. The surface is modelled as a thin layer of compliant dermis bound by stiff epidermis. This layer is injected with fluid with its inner surface constrained. Representing a rigid muscle and fat layer. The GPS changes associated with this pressurisation are then examined for general trends, and a derived metric is assessed against the remaining limbs, which were not subject to FEA. This approach reduces the computational expense of extensive FEA. While this approach relies on a simplified model of lymphoedema its observations are validated directly against patient data. The method by which this FEA model was implement is described below.

A two-layer steady-state biphasic model was implemented in $F E B I O$ (Scientific Computing and Imaging Institute, University of Utah, USA). The outer surface of the scans was modelled as two biphasic layers; the inner layer representing the dermal tissues, with a Young's modulus of $1.17 \mathrm{kPa}$ and a solid fraction of 0.2 and the outer layer representing the epidermis, with a Young's modulus of $1364 \mathrm{kPa}$ and a solid fraction of 0.8 , both with a Poisson ratio of 0.4 [107]. An increasing fluid pressure was applied in a steady-state manner to the internal surface, which was fixed in space. The geometries were meshed using linear hexahedral elements. Note that the permeability was irrelevant as in a steady-state simulation the model is at equilibrium. The meshes were coarsened three times by halving the number of elements each time and, subsequently, all meshes were subjected to a hydrostatic pressure of $3 \mathrm{kPa}$. It was found that for every case the difference in volume change between the two finest geometries was less than one percent. This reduction was necessary to reduce computational expense, after simulation the mesh was resampled at the original, finer, density before calculating the G2-redundancy. 


\subsection{Results}

\subsubsection{In-silico Study}

\subsubsection{Sensitivity to Noise}

In order to assess the sensitivity of G2-redundancy to noise, the three affected geometries used in the in-silico study had additive and multiplicative Gaussian noise imposed in increasing quantities and the resulting change in G2-redundancy calculated. The volume of each noisy geometry was also measured and the error calculated. This process was repeated 100 times for each of the three geometries, the mean and standard deviation of that change are shown in Figure 5.6. For small levels of noise, the zero-mean displace-
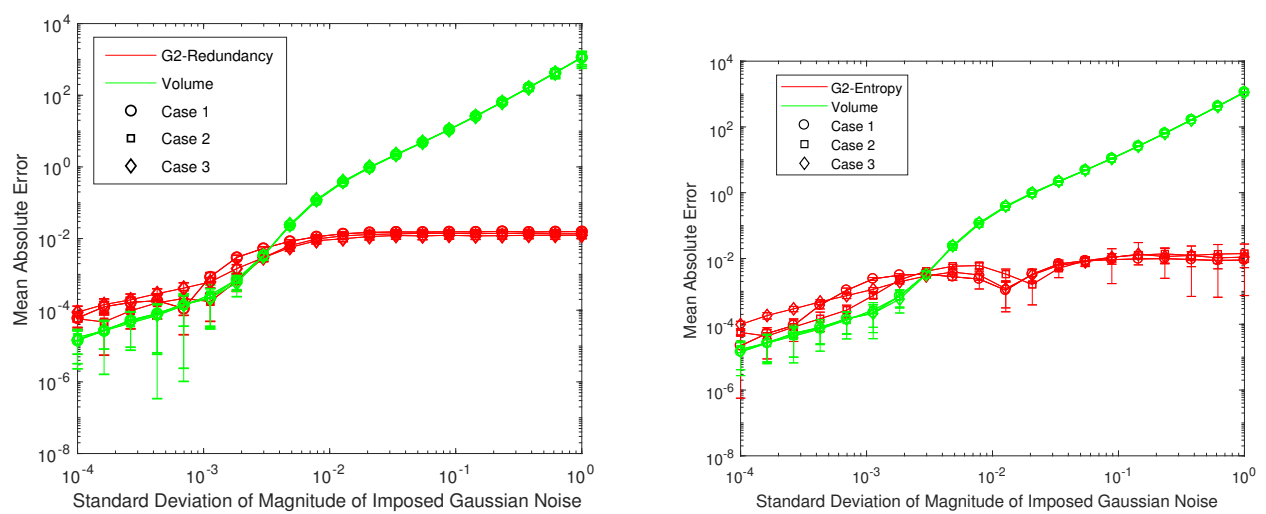

FiguRE 5.6: Relative error in G2-entropy and volume for each of the unaffected geometries removed from the original study with the presence of an additive (top) and multiplicative (bottom) Gaussian noise. The bars represent one standard deviation for 100 runs.

ment nature of the noise causes little variation in either metric yet volume outperforms G2-redundancy. However, at higher noise levels approximately 1-10\% G2-redundancy becomes more noise resistant for both additive and multiplicative noise. 


\subsubsection{Pressurisation}

Shown in Figure 5.8 is the volume change associated with pressurisation for each of the three unaffected limb geometries removed from the in-vivo study. As can be seen in cross-section also in Figure 5.8 the pressurisation swells the dermal tissues causing the the epidermis to progress toward a surface of minimum curvature. Shown in Figure 5.7 are the GPS-distance distributions for the unaffected geometries in each of the three in-silico cases at each pressure step. Each distribution is coloured from blue to red representing the amount of pressurisation, 0 to $3 \mathrm{kPa}$. As expected, the general change for all distributions is a reduction in the entropy associated with each distribution during pressurisation. Whilst the relationship in non-monotonic, particularly in case one. However, the buckling associated with this would not occur physiologically as the stiff epidermis would remodel and relax over the large time-scales associated with lymphoedema. Its is assumed that this phenomena can be ignored. As this pressurisation is used only to find spectral changes associated with lymphoedema that can be used to design a shape-based metric of lymphoedema. However, a more complex model of the tissues could explore this phenomenon.

The increase in the probability of a given distance coupled with a decrease in the range of observed distances reduced the amount of information associated with each point in the original geometry. This loss of information can be considered as a spectral homogenisation of the geometry. The change in G2-redundancy associated with pressurisation can be seen in Figure 5.8.

\subsubsection{In-vivo Study}

The G2-redundancy of the 21 patients within the in-vivo study was calculated and the G2-redundancy values were compared with both the affected and unaffected limbs. A statistically significant difference in the mean of the samples was identified. The G2redundancy means were found to be $7.17 \%$ (6.99-7.35, 95\% CI) and 6.73 (6.55-6.92, $95 \%$ CI) for unaffected and affected limbs respectively, $t(40)=10.51, p=0.0023$. To 

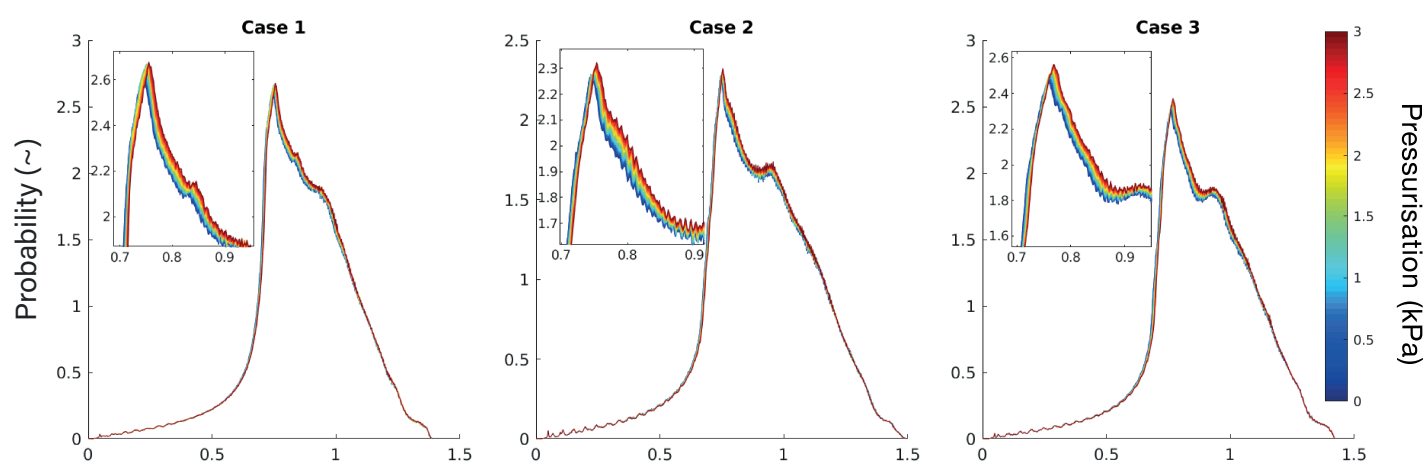

G2 Distance ( )

Figure 5.7: In-silico simulations illustrate the effect of limb swelling on the G2distributions for GPS series that are truncated from the Fiedler vector to the point of noise dominance. The colours represent the hydrostatic pressure in $\mathrm{kPa}$.

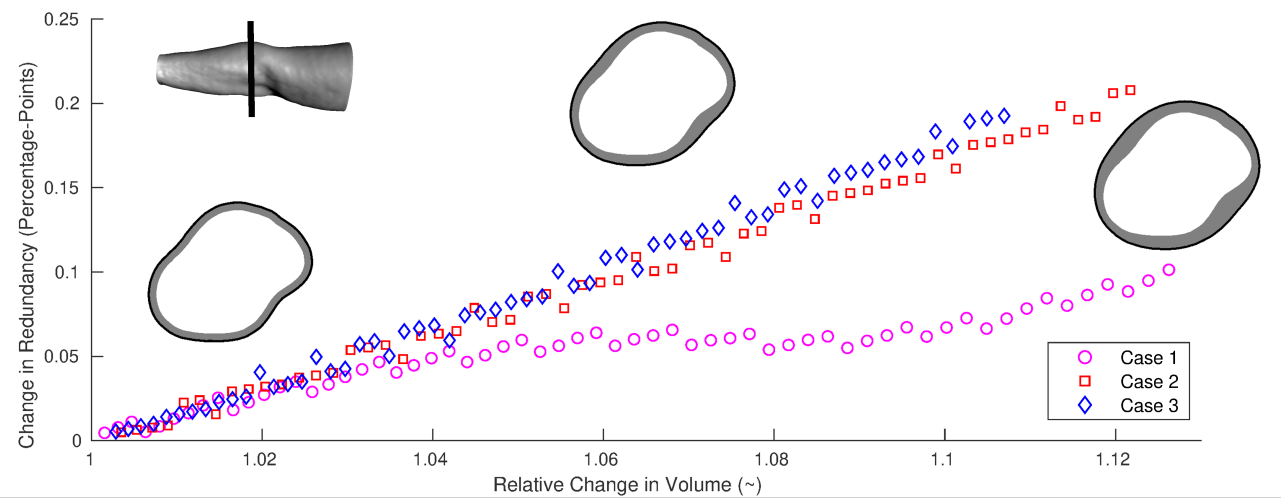

Figure 5.8: Redundancy change associated with pressurisation for each in-silico case. Also shown are cross-sections of case one for three applied loads.

assess the potential of G2-redundancy as a diagnostic criterion, an error minimising threshold for lymphoedema of $7.05 \%$ was found. If used to diagnose the individual limbs in this study, then it would have a sensitivity of 76.6 (58.5-94.7 95\% CI) and a specificity of $86.7 \%(72.2-100,95 \%$ CI). The threshold can be seen along with violin plots of G2-redundancy for affected and unaffected limbs in Figure 5.9. A similar plot is also shown for limb volume with means of $2.43 \mathrm{~L}(2.21-2.65,95 \% \mathrm{CI})$ and $2.13 \mathrm{~L}$ (1.97 to $2.29,95 \%$ CI) for affected and unaffected limbs respectively, $t(40)=4.38, p=0.0428$. No significant correlation was found between G2-redundancy and limb volume for affected or unaffected arms respectively, Pearson $\mathrm{r}(19)=0.258, \mathrm{p}=0.259$ and $\mathrm{r}(19)=0.239, \mathrm{p}=0.296$. A plot showing the difference in both metrics between affected and unaffected arms for 
each patient is shown in Figure 5.10.
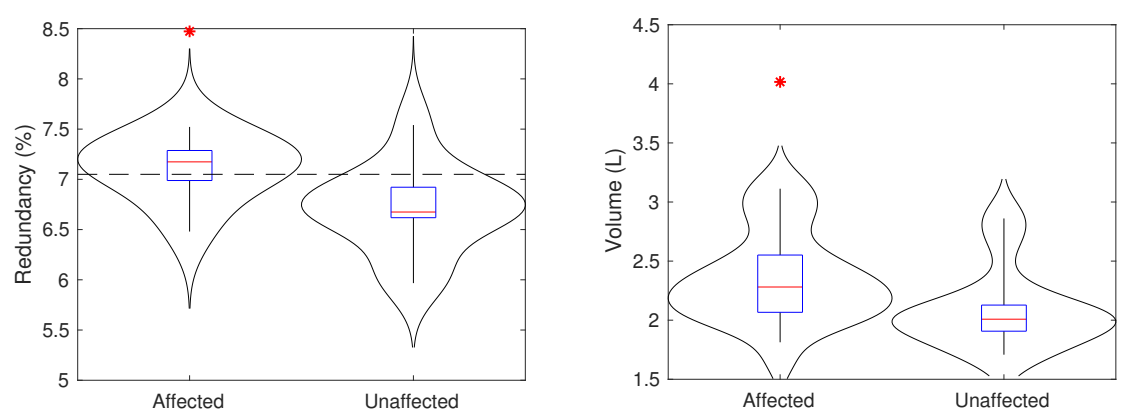

Figure 5.9: Violin plots for affected and unaffected limbs. Top: G2-redundancy. Bottom: volume. A significant difference exists for both. On the G2-redundancy plot a hypothetical threshold of $7.05 \%$ is shown as a horizontal dashed line.

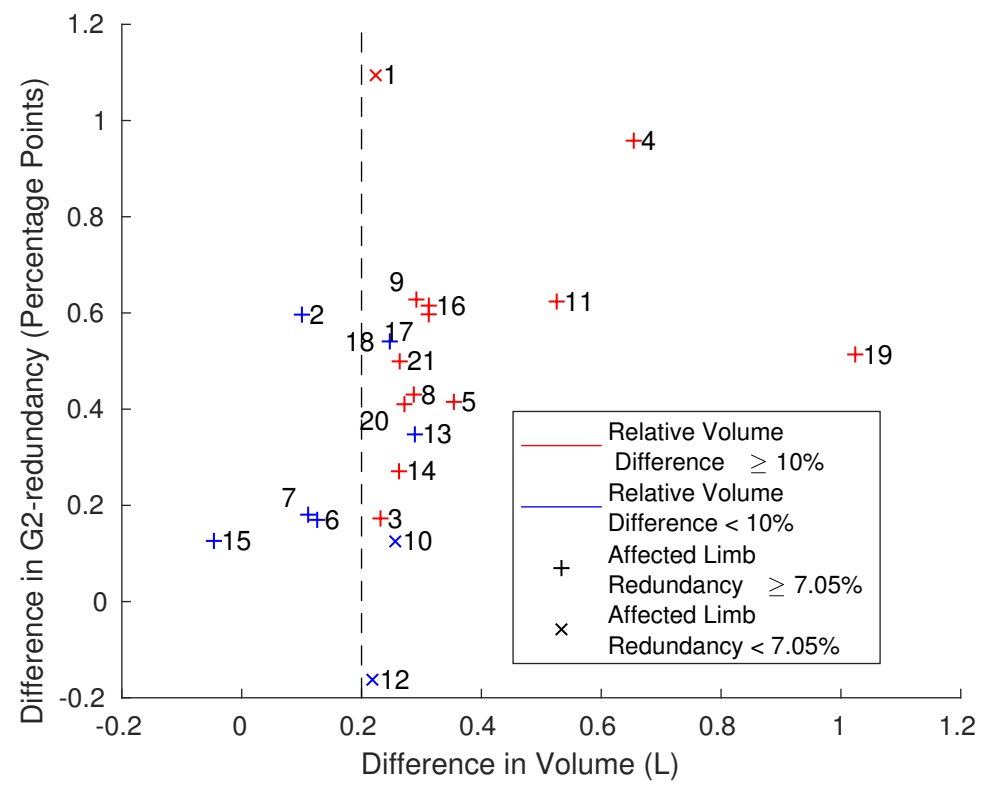

FIGURE 5.10: The signed difference between the affected and unaffected arm in G2redundancy and volume is plotted for each patient. Difference $=$ Affected-Unaffected. No statistically significant relationship was found between the two. 


\subsection{Discussion}

Whilst a statistically significant difference was observed in the affected vs unaffected means for both volume and G2-redundancy, there is a higher confidence in the relationship for G2-redundancy than there is for volume. The absence of a statistically significant relationship between limb volume and G2-redundancy suggests that this metric captures an aspect of shape which is independent of volume. Which implies that this metric could be used to allow greater understanding of disease state. As can be seen in Figure 5.10, some patients have large discrepancies between the values. For example, patient 19 has a $1 \mathrm{~L}$ difference in the volume between limbs, the largest observed, yet their G2-redundancy difference is within the 6th decile. Conversely, patient 2 has the second smallest observed difference in limb volume yet has a G2-redundancy difference comparable to patient 19. Further work should seek to relate these metrics to clinical outcomes to assess whether these discrepancies are of clinical value.

As can be seen in Figure 5.6 G2-entropy shows resistance to noise, with the standard deviation of error being smaller than the standard deviation of the applied noise. This is to be expected given the method of truncating the manifold harmonic transform such that high-frequency noise is rejected. Whilst volume is similarly robust to low-level additive noise due to the mean displacement of the nodes being zero, it is important to note that G2-entropy outperforms volume in the presence of large additive and multiplicative noise.

The assessment of entropy needs to be considered carefully in the use of a histogram method. Too coarse a sampling of the G2 range can over-smooth and obscure changes associated with lymphoedema whereas oversampling can emphasise noise over the underlying distribution.

If a threshold G2-redundancy of $7.05 \%$ were used as a criterion, considering the data-set series of limbs with and without lymphoedema, it would have a sensitivity of $76.6 \%(58.5-94.795 \% \mathrm{CI})$ and a specificity of $86.7 \%(72.2-100,95 \% \mathrm{CI})$, this is comparable, if not better, in performance to criteria defined as differences between limbs for currently 


\begin{tabular}{lcc}
\hline $\begin{array}{l}\text { Diagnostic } \\
\text { criteria }\end{array}$ & $\begin{array}{c}\text { Sensitivity } \\
(95 \% \mathrm{CI})\end{array}$ & $\begin{array}{c}\text { Specificity } \\
(95 \% \mathrm{CI})\end{array}$ \\
\hline $\begin{array}{l}\text { Volume Difference } \\
\quad \geq 200 \mathrm{ml}\end{array}$ & $0.39(0.27-0.51)$ & $1.00(0.95-1.00)$ \\
$\quad \geq 4 \%$ & $0.67(0.55-0.78)$ & $0.89(0.79-0.95)$ \\
$\begin{array}{l}\text { Circumference Difference } \\
\quad \geq 2 \mathrm{~cm}\end{array}$ & $0.36(0.25-0.48)$ & $0.94(0.86-0.98)$ \\
$\begin{array}{l}\text { Bioimpedance } \\
\quad \geq 1 \text { SD }\end{array}$ & $0.87(0.77-0.94)$ & $0.47(0.35-0.56)$ \\
$\geq 2$ SD & $0.51(0.39-0.64)$ & $0.99(0.92-1.00)$ \\
\hline
\end{tabular}

TABLE 5.3: Published diagnostic sensitivities and specificities for upper-limb breast cancer related lymphoedema [8]

available techniques, see Table 5.3 [108][8]. This is not to say that these values would be found in a pre-diagnosis population but it is indicative of the ability of this metric to diagnose lymphoedema based on the intrinsic shape of a limb, not in comparison to an unaffected limb. As far as the author is aware, there is no quantitative metric of lymphoedema that does not require the comparison between affected and unaffected limbs. This raises the possibility that this technique could be used to aid the diagnosis of lymphoedema when limbs cannot be compared, future work should seek to explore this.

\subsection{Conclusion}

A volume-independent shape-based metric for assessing lymphoedema has been proposed and evaluated against a sample of unilateral lymphoedema patients. A statistically significant correlation between G2 entropy and the presence of lymphoedema was found. As the quantification of volume offered benefit to the definition of swelling in lymphoedema. The quantification of shape may offer similar benefit both in diagnosis through a quantification of the aims of CTCv3 and further allowing the reversal of shape changes to be quantified when evaluating the efficiency of treatment. 
Many other phenomena play a role in limb shape such as age, hydration, obesity, and activity. Additionally, many unconsidered phenomena will effect the change in shape associated with lymphoedema such as changes in skin compliance, tissue remodelling, and changes in lymphatic capacity associated with the change in downstream pressure condition. The current study would appear to validate the decision to omit these behaviours as a statistically significant relationship was found, for this patient population, without a metric designed with these behaviours in mind. Rather than include these in the FEA modelling producing considerably more complex models and using these to adjust the metric; the authors propose that future work seeks to apply this metric to wider populations and debate any observed inconsistencies. Further work should seek to address whether this metric could augment volume-based methods in the diagnosis or treatment planning of lymphoedema and to establish the relationship between the severity of lymphoedema and G2-entropy. Further development of the methodology could explore more advanced methods to assess the entropy of the G2distributions instead of a histogram approach. This may increase the sensitivity of the method to lymphoedema without affecting sensitivity to discretisation. The work undertaken in Chapter 4 raises the possibility that specific injuries could be associated to specific shape changes. However, this would require coupling a 0D lumped model of the lymphatic network to a biphasic model of the tissues, and a mechanism for incorporating remodelling phenomena, future work should seek to address this. 



\section{Chapter 6}

\section{Concluding Remarks}

This work set out to numerically model the mechanics of lymph transport, with the aim of providing insight into lymphatic disease, formulating hypotheses of lymphatic physiology and identifying potential new avenues of research. Starting with a 3D analysis of a single subject-specific lymphatic valve. A mechanism was presented for previous experimental results showing the effect of trans-mural pressure on the pressure required to close lymphatic valves and the importance of wall motion in future FSI studies of lymphatic valve dynamics were identified.

The potential benefits of expanding the scale of lymphatic modelling were identified and a consideration of the steps necessary was undertaken. 0D Lumped modelling was identified as a way to reduce computational expense, previous approaches to lumped modelling were considered and modifications were proposed. A less-idealised valve model, incorporating trans-mural dependent bias, was proposed as well as a method of allowing self-organised contraction through a stretch-dependent frequency of contraction.

A network of the superficial lymphatics of the upper-limb was reconstructed from an anatomical sketch. The network was used in conjunction with the lumped model to produce a 421 vessel lymphatic model consisting of 17,706 lymphangions. The 
first lymphangion scale model which considered more than seven vessels. An attempt to study the fluid flows within this network revealed issues with the coordination of contractions at confluences which frustrated further progress. However, a potential metric of insufficiency based on the modality of the distribution of contraction durations and the reduce efficiency of contractions at higher adventitious pressure differences was identified.

A simplified patient-specific biphasic model of lymphoedema was proposed and used to develop a novel shape-based metric for lymphoedema. A statistically significant relationship between the metric and the presence of lymphoedema was found.

There are missed opportunities in the current state of the art that if undertaken could improve understanding and help convert current work into methods which improve patient outcomes. Firstly, there is limited understanding of the mechanics of lymphatic wall motion. In 2010, Arkil et al noted it was strange that the field had persisted for so long without such an understanding [36]. The subsequent decade has not yielded a constitutive model of the lymphangion wall and has done little to progress toward such a model. 3D computational models of valve behaviour, including this work continue to develop but offer limited insight until the mechanics of the wall are well understood. Secondly, a variety of species and sites make up the landmark studies that have discovered the phenomena which drive lymphatic pumping. These studies are almost universally not repeated in other species or at other sites.

Lumped modelling of lymphatics has benefits beyond those considered in this work. The ability to use large-scale lumped models of the lymphatic system, such as that presented in this work, to model the metastasis of cancer and the development of immunity could have profound implications for the treatment and prevention of disease. However, such efforts are hampered by the mixture of bovine, murine, rat and bat experimental models in the thoracic duct, mesentery and limb which make up the current state of the art. Future work toward coupling lumped models of the lymphatic system and biphasic models of the tissues could lead to allow more insight into the relationship between lymphatic injury and the development of lymphoedema. 


\section{Appendix A}

\section{Kálmán Filter Overview}

The ensemble Kálmán filter draws multiple observations of the state variables from a distribution, propagates these observations in time, then calculates the mean and covariance empirically. A function $G$ is defined to map bound state variable from unbound values such that the state can be considered $[-\infty, \infty]$ which avoids the complication of calculating the mean and covariance of a truncated multi-variate distribution by empirical observation.

$$
\begin{gathered}
G: R^{n_{p}}\left[\boldsymbol{r}_{\mathrm{high}}, \boldsymbol{r}_{\text {low }}\right] \rightarrow R^{n_{p}} \\
\overline{\boldsymbol{\Xi}}=G(\boldsymbol{\Xi})=\frac{1}{1+\exp (5 \boldsymbol{\Xi})}\left(\boldsymbol{r}_{\text {high }}-\boldsymbol{r}_{\text {low }}\right)+\boldsymbol{r}_{\text {low }} ;
\end{gathered}
$$

With $\boldsymbol{\Xi}$ representing the bound and $\overline{\boldsymbol{\Xi}}$ representing the unbound state. With $\boldsymbol{r}_{\text {high }}$ and $\boldsymbol{r}_{\text {low }}$ representing the range of values for $\boldsymbol{\Xi}$.

$$
\overline{\boldsymbol{\Xi}} \sim \mathcal{N}_{n_{p}, n_{o}}\left(\boldsymbol{\mu},{ }^{n} \mathbf{P}_{\boldsymbol{x} \boldsymbol{x}}\right)
$$

$\bar{\Xi}$ consists of $n_{o}$ members drawn from a $n_{p}$-variate Gaussian distribution whose mean is represented by the vector $\boldsymbol{\mu}$ and has the covariance ${ }^{n} \mathbf{P}_{\boldsymbol{x} \boldsymbol{x}}$

$$
\hat{\mathbf{x}}=\frac{1}{n_{o}} \sum(\boldsymbol{\Xi})=\frac{1}{n_{o}} \sum\left(G^{-1}(\overline{\boldsymbol{\Xi}})\right)
$$


The mean state $\hat{\mathbf{x}}$

$$
\hat{\mathbf{y}}=\frac{1}{n_{o}} \sum(\dagger)
$$

The state variables are propagated, in time, through the model.

$$
\mathbf{x}=f\left(\bar{\Xi}, t_{n}\right)
$$

After propagation the state variable can be transformed into the observation vector which will be compared to experimental data.

$$
\dagger=\mathcal{H}(\mathbf{x})
$$

The difference from the mean state and observation for each sample can be can be calculated

$$
\begin{aligned}
& e_{x}=\mathrm{x}-\hat{\mathrm{x}} \\
& e_{y}=\dagger-\hat{\mathrm{y}}
\end{aligned}
$$

From this the covariances ${ }^{n+1} \mathbf{P}_{\boldsymbol{y} \boldsymbol{y}}$ and ${ }^{n+1} \mathbf{P}_{\boldsymbol{x} \boldsymbol{y}}$ can be calculated

$$
\begin{aligned}
& { }^{n+1} \mathbf{P}_{\boldsymbol{y} \boldsymbol{y}}=\mathbf{e}_{\mathbf{y}} \mathbf{e}_{\mathbf{y}}{ }^{\mathrm{T}} \\
& { }^{n+1} \mathbf{P}_{\boldsymbol{x} \boldsymbol{y}}=\mathbf{e}_{\mathbf{x}} \mathbf{e}_{\mathbf{y}}{ }^{\mathrm{T}}
\end{aligned}
$$

The filter gain can now be found

$$
\mathbf{K}={ }^{n+1} \mathbf{P}_{\boldsymbol{x} \boldsymbol{y}}{ }^{n+1} \mathbf{P}_{\boldsymbol{y} \boldsymbol{y}}^{-1}
$$

This allows the error in mean observation to be used to adjust the mean state.

$$
\boldsymbol{\mu}=\hat{\boldsymbol{x}}+\mathbf{K}\left(\hat{\boldsymbol{y}}-\boldsymbol{y}_{\text {exp }}\right)
$$


Similarly the covariance in observation can be used to correct the covariance in state.

$$
{ }^{n+1} \mathbf{P}_{\boldsymbol{x} \boldsymbol{x}}={ }^{n+1} \mathbf{P}_{\boldsymbol{x} \boldsymbol{x}}^{-}-\mathbf{K} *{ }^{n+1} \mathbf{P}_{\boldsymbol{y} \boldsymbol{y}} * \mathbf{K}^{\mathrm{T}}
$$





\section{Appendix B}

\section{Contraction Function Derivation}

Assume that some function whose gradient is directly proportional to its state and alternates between two modes in time.

$$
\frac{d \hat{M}}{d t}=\left\{\begin{array}{l}
k_{c}(\hat{M(t)}-1), 0 \leq t<t_{c} \\
-k_{r} \hat{M(t)}, t_{c} \leq t<t_{r}
\end{array}\right.
$$

Boundary conditions

$$
\begin{aligned}
& \hat{M(0)}=\hat{M}_{b} \\
& M \hat{\left(t_{c}\right)}=\hat{M}_{u}
\end{aligned}
$$

solving the contraction

$$
\begin{gathered}
\log \left|\frac{M(t)-1}{M_{b}-1}\right|=k_{c} t \\
\log \left|\frac{M(t)}{M_{u}}\right|=k_{r}\left(t_{c}-t\right)
\end{gathered}
$$

find $t_{c}, M\left(t_{c}\right)=M_{u}$ for eq. B.4

$$
\frac{1}{k_{c}} \log \left|\frac{1-M_{u}}{1-M_{b}}\right|=t_{c}
$$


find $t_{r}, M\left(t_{r}\right)=M_{b}$ from eq. B.5 and eq. B.6

$$
\frac{1}{k_{c}} \log \left|\frac{1-M_{u}}{1-M_{b}}\right|-\frac{1}{k_{r}} \log \left|\frac{M_{b}}{M_{u}}\right|=t_{r}
$$

Introduce the contraction parameters

$$
\begin{gathered}
T=t_{c}+t_{r}=\frac{2}{k_{c}} \log \left|\frac{1-M_{u}}{1-M_{b}}\right|-\frac{1}{k_{r}} \log \left|\frac{M_{b}}{M_{u}}\right| \\
M_{\max }=M_{0} M_{u} \\
M_{\min }=M_{0} M_{b} \\
\phi=\frac{t_{c}}{t_{r}}=\frac{\log \left|\frac{1-M_{u}}{1-M_{b}}\right|}{\log \left|\frac{1-M_{u}}{1-M_{b}}\right|-\frac{k_{c}}{k_{r}} \log \left|\frac{M_{b}}{M_{u}}\right|}
\end{gathered}
$$

Isolate $k_{c}, k_{r}$ from B.11.

$$
\log _{\frac{M_{b}}{M_{u}}}\left|\frac{1-M_{u}}{1-M_{b}}\right| \frac{\phi-1}{\phi}=\frac{k_{c}}{k_{r}}
$$

substitute $k_{r}$ into B.8

$$
k_{c}=\frac{\phi+1}{T \phi} \log \left|\frac{1-M_{u}}{1-M_{b}}\right|
$$

substitute B.13 into B.12

$$
k_{r}=\frac{\phi+1}{T(\phi-1)} \log \left|\frac{M_{b}}{M_{u}}\right|
$$




\section{Appendix C}

\section{Upper Limb Network}

Presented below is the lymphatic network produced in Section 3.5.1. The following table presented in eleven parts lists each vessel, it's length, starting and ending positions and starting and ending diameter. The following table, presented in eleven parts, shows the connectivity between vessels with the end of each parent vessel being connected to the corresponding daughter vessel. 


\begin{tabular}{|c|c|c|c|c|c|c|c|c|c|}
\hline \multirow[t]{2}{*}{ Vessel Number } & \multirow{2}{*}{$\frac{\text { Length }}{(\mathrm{cm})}$} & \multicolumn{3}{|c|}{ Starting Position $(\mathrm{cm})$} & \multicolumn{3}{|c|}{ Ending Position $(\mathrm{cm})$} & \multirow{2}{*}{$\frac{\text { Starting }}{\text { Diameter }(\mu m)}$} & \multirow{2}{*}{$\begin{array}{c}\text { Ending } \\
\text { Diameter }(\mu m)\end{array}$} \\
\hline & & $x$ & $y$ & $z$ & $x$ & $y$ & $z$ & & \\
\hline 1 & 5.505 & -4.798 & 3.278 & 21.299 & -4.798 & 3.278 & 21.299 & 293.699 & 316.802 \\
\hline 2 & 4.176 & -3.351 & 4.010 & 24.536 & -3.351 & 4.010 & 24.536 & 459.356 & 442.848 \\
\hline 3 & 2.770 & -5.295 & 2.657 & 25.586 & -5.295 & 2.657 & 25.586 & 439.292 & 148.695 \\
\hline 4 & 1.732 & -5.936 & 1.946 & 25.499 & -5.936 & 1.946 & 25.499 & 129.173 & 148.695 \\
\hline 5 & 1.001 & -5.070 & 2.680 & 25.999 & -5.070 & 2.680 & 25.999 & 244.710 & 156.928 \\
\hline 6 & 3.478 & -6.300 & 1.405 & 23.986 & -6.300 & 1.405 & 23.986 & 342.223 & 284.077 \\
\hline 7 & 1.595 & -6.593 & 0.223 & 24.199 & -6.593 & 0.223 & 24.199 & 294.499 & 282.507 \\
\hline 8 & 2.844 & -6.580 & -0.353 & 21.999 & -6.580 & -0.353 & 21.999 & 406.305 & 350.517 \\
\hline 9 & 1.886 & -6.581 & 0.349 & 25.299 & -6.581 & 0.349 & 25.299 & 189.291 & 148.695 \\
\hline 10 & 1.023 & -6.532 & 0.639 & 25.699 & -6.532 & 0.639 & 25.699 & 129.173 & 148.695 \\
\hline 11 & 1.764 & -6.541 & -0.595 & 25.599 & -6.541 & -0.595 & 25.599 & 165.988 & 148.695 \\
\hline 12 & 8.161 & -1.794 & -4.595 & 20.399 & -1.794 & -4.595 & 20.399 & 186.642 & 244.967 \\
\hline 13 & 2.141 & -1.580 & 4.309 & 25.736 & -1.580 & 4.309 & 25.736 & 405.229 & 448.640 \\
\hline 14 & 4.325 & -5.446 & 2.007 & 26.949 & -5.446 & 2.007 & 26.949 & 408.260 & 467.052 \\
\hline 15 & 1.954 & -5.736 & 1.353 & 27.999 & -5.736 & 1.353 & 27.999 & 296.199 & 227.310 \\
\hline 16 & 2.845 & -6.272 & -0.486 & 26.649 & -6.272 & -0.486 & 26.649 & 363.592 & 348.250 \\
\hline 17 & 1.971 & -5.734 & -1.489 & 27.399 & -5.734 & -1.489 & 27.399 & 149.847 & 172.494 \\
\hline 18 & 1.779 & -5.589 & -1.998 & 26.549 & -5.589 & -1.998 & 26.549 & 329.557 & 436.681 \\
\hline 19 & 3.735 & -4.953 & -3.090 & 24.349 & -4.953 & -3.090 & 24.349 & 400.692 & 148.695 \\
\hline 20 & 3.657 & -3.146 & -3.998 & 25.249 & -3.146 & -3.998 & 25.249 & 400.692 & 256.956 \\
\hline 21 & 7.114 & -5.998 & 0.177 & 29.749 & -5.998 & 0.177 & 29.749 & 276.875 & 400.236 \\
\hline 22 & 6.074 & -2.164 & -3.606 & 27.649 & -2.164 & -3.606 & 27.649 & 307.755 & 255.466 \\
\hline 23 & 1.515 & -5.929 & -0.506 & 30.099 & -5.929 & -0.506 & 30.099 & 192.308 & 148.695 \\
\hline 24 & 1.472 & -5.702 & -1.037 & 30.949 & -5.702 & -1.037 & 30.949 & 181.471 & 148.695 \\
\hline 25 & 5.195 & -2.654 & -3.231 & 29.499 & -2.654 & -3.231 & 29.499 & 318.178 & 305.222 \\
\hline 26 & 2.299 & -1.559 & 3.726 & 27.799 & -1.559 & 3.726 & 27.799 & 405.312 & 349.627 \\
\hline 27 & 4.648 & -4.228 & 2.478 & 32.324 & -4.228 & 2.478 & 32.324 & 326.144 & 300.702 \\
\hline 28 & 1.624 & -5.139 & 1.853 & 32.799 & -5.139 & 1.853 & 32.799 & 287.226 & 148.695 \\
\hline 29 & 3.039 & -5.342 & 1.726 & 34.149 & -5.342 & 1.726 & 34.149 & 251.733 & 179.183 \\
\hline 30 & 1.101 & -5.069 & 1.886 & 32.199 & -5.069 & 1.886 & 32.199 & 199.584 & 148.695 \\
\hline 31 & 2.581 & -5.722 & 1.276 & 34.249 & -5.722 & 1.276 & 34.249 & 214.574 & 203.684 \\
\hline 32 & 4.423 & -4.935 & 1.979 & 30.861 & -4.935 & 1.979 & 30.861 & 328.243 & 302.429 \\
\hline 33 & 2.514 & -5.963 & 0.370 & 31.649 & -5.963 & 0.370 & 31.649 & 390.649 & 305.822 \\
\hline 34 & 1.119 & -5.991 & 0.273 & 31.249 & -5.991 & 0.273 & 31.249 & 159.222 & 148.695 \\
\hline 35 & 1.146 & -6.034 & -0.095 & 32.099 & -6.034 & -0.095 & 32.099 & 249.258 & 343.410 \\
\hline 36 & 0.447 & -6.035 & -0.073 & 32.299 & -6.035 & -0.073 & 32.299 & 277.832 & 180.919 \\
\hline 37 & 2.110 & -5.527 & -1.397 & 32.136 & -5.527 & -1.397 & 32.136 & 203.187 & 174.336 \\
\hline 38 & 0.575 & -5.522 & -1.449 & 32.499 & -5.522 & -1.449 & 32.499 & 223.564 & 148.695 \\
\hline 39 & 1.259 & -5.053 & -1.904 & 31.749 & -5.053 & -1.904 & 31.749 & 230.874 & 257.832 \\
\hline 40 & 2.046 & -5.726 & 1.162 & 32.649 & -5.726 & 1.162 & 32.649 & 469.027 & 378.829 \\
\hline
\end{tabular}




\begin{tabular}{|c|c|c|c|c|c|c|c|c|c|}
\hline Vessel Number & Length & Starti & g Positic & $(\mathrm{cm})$ & Endin & Positic & $(\mathrm{cm})$ & Starting & Ending \\
\hline & $(\mathrm{cm})$ & $x$ & $y$ & $z$ & $x$ & $y$ & $z$ & Diameter $(\mu m)$ & Diameter $(\mu m)$ \\
\hline 41 & 6.460 & -3.171 & -2.956 & 31.949 & -3.171 & -2.956 & 31.949 & 262.393 & 377.562 \\
\hline 42 & 2.928 & -6.163 & 0.325 & 35.349 & -6.163 & 0.325 & 35.349 & 331.082 & 334.265 \\
\hline 43 & 2.216 & -6.028 & -0.794 & 35.649 & -6.028 & -0.794 & 35.649 & 350.605 & 264.973 \\
\hline 44 & 2.141 & -6.187 & 0.095 & 36.899 & -6.187 & 0.095 & 36.899 & 269.214 & 237.684 \\
\hline 45 & 16.508 & -5.562 & -0.588 & 46.874 & -5.562 & -0.588 & 46.874 & 362.006 & 384.195 \\
\hline 46 & 7.515 & -4.854 & 2.182 & 37.211 & -4.854 & 2.182 & 37.211 & 357.984 & 307.152 \\
\hline 47 & 6.107 & -5.873 & 0.646 & 42.049 & -5.873 & 0.646 & 42.049 & 180.132 & 148.695 \\
\hline 48 & 5.200 & -5.514 & 1.184 & 42.536 & -5.514 & 1.184 & 42.536 & 338.141 & 377.624 \\
\hline 49 & 1.666 & -5.501 & 0.993 & 43.899 & -5.501 & 0.993 & 43.899 & 317.163 & 187.841 \\
\hline 50 & 4.854 & -5.639 & -0.347 & 46.999 & -5.639 & -0.347 & 46.999 & 336.276 & 359.413 \\
\hline 51 & 2.781 & -5.898 & 0.179 & 49.524 & -5.898 & 0.179 & 49.524 & 418.893 & 215.648 \\
\hline 52 & 5.982 & -3.617 & 2.861 & 36.499 & -3.617 & 2.861 & 36.499 & 283.199 & 201.581 \\
\hline 53 & 6.078 & -5.934 & -0.767 & 40.049 & -5.934 & -0.767 & 40.049 & 335.302 & 468.067 \\
\hline 54 & 3.408 & -5.864 & 0.439 & 42.749 & -5.864 & 0.439 & 42.749 & 417.507 & 189.320 \\
\hline 55 & 5.194 & -5.531 & -0.671 & 46.811 & -5.531 & -0.671 & 46.811 & 325.073 & 299.363 \\
\hline 56 & 6.081 & -5.512 & -0.721 & 46.805 & -5.512 & -0.721 & 46.805 & 348.269 & 400.905 \\
\hline 57 & 3.070 & -5.945 & -0.302 & 49.886 & -5.945 & -0.302 & 49.886 & 255.947 & 294.630 \\
\hline 58 & 6.836 & -5.596 & -1.158 & 41.899 & -5.596 & -1.158 & 41.899 & 332.647 & 197.011 \\
\hline 59 & 13.679 & -5.610 & 0.841 & 48.949 & -5.610 & 0.841 & 48.949 & 391.030 & 270.077 \\
\hline 60 & 1.904 & -0.902 & 3.407 & 39.511 & -0.902 & 3.407 & 39.511 & 388.807 & 422.784 \\
\hline 61 & 4.945 & -3.853 & 2.328 & 43.261 & -3.853 & 2.328 & 43.261 & 458.558 & 269.953 \\
\hline 62 & 1.616 & -2.174 & 3.233 & 39.261 & -2.174 & 3.233 & 39.261 & 247.807 & 335.664 \\
\hline 63 & 1.642 & -1.315 & 3.425 & 38.299 & -1.315 & 3.425 & 38.299 & 241.985 & 167.134 \\
\hline 64 & 2.584 & -3.735 & 2.620 & 40.549 & -3.735 & 2.620 & 40.549 & 336.149 & 207.981 \\
\hline 65 & 1.919 & 0.022 & 3.202 & 41.961 & 0.022 & 3.202 & 41.961 & 445.652 & 430.039 \\
\hline 66 & 3.037 & -2.222 & 2.968 & 41.949 & -2.222 & 2.968 & 41.949 & 324.152 & 148.695 \\
\hline 67 & 5.017 & -2.747 & 2.614 & 45.999 & -2.747 & 2.614 & 45.999 & 386.679 & 354.463 \\
\hline 68 & 4.529 & -3.671 & 2.342 & 47.349 & -3.671 & 2.342 & 47.349 & 310.511 & 294.753 \\
\hline 69 & 3.023 & -4.376 & 1.906 & 46.274 & -4.376 & 1.906 & 46.274 & 302.684 & 273.331 \\
\hline 70 & 1.241 & -4.908 & 1.520 & 46.349 & -4.908 & 1.520 & 46.349 & 461.251 & 519.117 \\
\hline 71 & 2.441 & -5.576 & 0.611 & 47.349 & -5.576 & 0.611 & 47.349 & 402.882 & 396.113 \\
\hline 72 & 1.404 & 0.018 & 3.008 & 44.599 & 0.018 & 3.008 & 44.599 & 222.026 & 285.749 \\
\hline 73 & 2.111 & -1.414 & 2.908 & 44.761 & -1.414 & 2.908 & 44.761 & 341.845 & 224.335 \\
\hline 74 & 1.975 & -1.746 & 2.841 & 45.949 & -1.746 & 2.841 & 45.949 & 129.173 & 148.695 \\
\hline 75 & 1.148 & -1.275 & 2.962 & 44.099 & -1.275 & 2.962 & 44.099 & 171.735 & 148.695 \\
\hline 76 & 0.731 & -1.943 & 2.836 & 44.749 & -1.943 & 2.836 & 44.749 & 214.488 & 148.695 \\
\hline 77 & 3.533 & -0.892 & 3.099 & 47.611 & -0.892 & 3.099 & 47.611 & 351.346 & 310.196 \\
\hline 78 & 2.481 & -4.979 & 1.596 & 48.224 & -4.979 & 1.596 & 48.224 & 427.378 & 630.028 \\
\hline 79 & 2.232 & -2.389 & 2.941 & 48.374 & -2.389 & 2.941 & 48.374 & 430.866 & 409.000 \\
\hline 80 & 1.215 & -3.081 & 2.577 & 47.436 & -3.081 & 2.577 & 47.436 & 374.067 & 408.504 \\
\hline
\end{tabular}




\begin{tabular}{|c|c|c|c|c|c|c|c|c|c|}
\hline \multirow[t]{2}{*}{ Vessel Number } & \multirow{2}{*}{$\frac{\text { Length }}{(\mathrm{cm})}$} & \multicolumn{3}{|c|}{ Starting Position $(\mathrm{cm})$} & \multicolumn{3}{|c|}{ Ending Position $(\mathrm{cm})$} & \multirow{2}{*}{$\frac{\text { Starting }}{\text { Diameter }(\mu m)}$} & \multirow{2}{*}{$\frac{\text { Ending }}{\text { Diameter }(\mu m)}$} \\
\hline & & $x$ & $y$ & $z$ & $x$ & $y$ & $z$ & & \\
\hline 81 & 3.087 & -2.362 & 3.224 & 50.111 & -2.362 & 3.224 & 50.111 & 322.023 & 308.435 \\
\hline 82 & 1.138 & -2.804 & 2.749 & 47.999 & -2.804 & 2.749 & 47.999 & 187.619 & 193.173 \\
\hline 83 & 1.801 & -3.406 & 2.716 & 48.999 & -3.406 & 2.716 & 48.999 & 383.559 & 418.871 \\
\hline 84 & 15.683 & -2.796 & 2.242 & 69.461 & -2.796 & 2.242 & 69.461 & 421.010 & 323.093 \\
\hline 85 & 5.800 & -6.218 & -0.348 & 51.474 & -6.218 & -0.348 & 51.474 & 257.288 & 400.392 \\
\hline 86 & 2.541 & 0.701 & 4.072 & 54.724 & 0.701 & 4.072 & 54.724 & 255.519 & 248.927 \\
\hline 87 & 1.866 & 0.912 & 4.147 & 56.474 & 0.912 & 4.147 & 56.474 & 298.554 & 343.676 \\
\hline 88 & 1.982 & 0.959 & 4.142 & 56.486 & 0.959 & 4.142 & 56.486 & 316.910 & 415.943 \\
\hline 89 & 9.452 & -1.976 & 2.788 & 67.349 & -1.976 & 2.788 & 67.349 & 380.192 & 326.206 \\
\hline 90 & 9.186 & -1.312 & 2.984 & 67.036 & -1.312 & 2.984 & 67.036 & 129.173 & 404.103 \\
\hline 91 & 0.799 & -1.785 & 2.847 & 67.246 & -1.785 & 2.847 & 67.246 & 370.693 & 301.735 \\
\hline 92 & 5.199 & -1.464 & 2.436 & 70.449 & -1.464 & 2.436 & 70.449 & 217.801 & 148.695 \\
\hline 93 & 3.370 & -0.270 & 2.610 & 70.011 & -0.270 & 2.610 & 70.011 & 352.118 & 286.615 \\
\hline 94 & 3.527 & -0.475 & 2.283 & 72.999 & -0.475 & 2.283 & 72.999 & 167.322 & 231.489 \\
\hline 95 & 6.384 & -0.728 & 1.900 & 78.161 & -0.728 & 1.900 & 78.161 & 210.699 & 271.171 \\
\hline 96 & 5.454 & -3.213 & 1.400 & 74.411 & -3.213 & 1.400 & 74.411 & 309.266 & 259.326 \\
\hline 97 & 2.014 & -3.647 & 1.598 & 71.211 & -3.647 & 1.598 & 71.211 & 315.582 & 242.185 \\
\hline 98 & 2.711 & -2.195 & 1.997 & 73.099 & -2.195 & 1.997 & 73.099 & 358.328 & 412.484 \\
\hline 99 & 1.581 & -3.443 & 1.550 & 72.399 & -3.443 & 1.550 & 72.399 & 344.042 & 303.748 \\
\hline 100 & 2.826 & -4.621 & 0.858 & 70.299 & -4.621 & 0.858 & 70.299 & 286.090 & 337.330 \\
\hline 101 & 2.321 & -0.151 & 1.808 & 79.899 & -0.151 & 1.808 & 79.899 & 207.800 & 302.574 \\
\hline 102 & 4.650 & 0.698 & 1.666 & 83.424 & 0.698 & 1.666 & 83.424 & 241.506 & 278.007 \\
\hline 103 & 10.024 & 0.543 & 1.612 & 87.162 & 0.543 & 1.612 & 87.162 & 254.160 & 402.103 \\
\hline 104 & 2.996 & -2.785 & 1.494 & 75.989 & -2.785 & 1.494 & 75.989 & 311.283 & 379.337 \\
\hline 105 & 5.381 & 0.706 & 3.783 & 60.849 & 0.706 & 3.783 & 60.849 & 396.554 & 358.098 \\
\hline 106 & 16.133 & -0.501 & -1.695 & 85.008 & -0.501 & -1.695 & 85.008 & 230.238 & 464.902 \\
\hline 107 & 7.269 & 1.149 & -1.543 & 85.124 & 1.149 & -1.543 & 85.124 & 306.374 & 241.935 \\
\hline 108 & 2.436 & -6.066 & -1.582 & 58.324 & -6.066 & -1.582 & 58.324 & 340.296 & 384.731 \\
\hline 109 & 13.467 & -2.941 & -0.977 & 78.811 & -2.941 & -0.977 & 78.811 & 253.631 & 344.082 \\
\hline 110 & 3.326 & -5.959 & -1.675 & 54.724 & -5.959 & -1.675 & 54.724 & 445.016 & 362.232 \\
\hline 111 & 2.088 & -6.332 & -1.187 & 56.824 & -6.332 & -1.187 & 56.824 & 243.119 & 279.863 \\
\hline 112 & 1.784 & -6.072 & -1.574 & 58.336 & -6.072 & -1.574 & 58.336 & 281.907 & 387.182 \\
\hline 113 & 5.201 & -1.939 & -1.330 & 82.500 & -1.939 & -1.330 & 82.500 & 281.434 & 259.175 \\
\hline 114 & 3.549 & -5.854 & -1.523 & 52.274 & -5.854 & -1.523 & 52.274 & 401.982 & 421.007 \\
\hline 115 & 2.790 & -6.335 & -1.051 & 54.774 & -6.335 & -1.051 & 54.774 & 387.286 & 460.016 \\
\hline 116 & 2.047 & -6.572 & -0.412 & 56.824 & -6.572 & -0.412 & 56.824 & 184.475 & 300.316 \\
\hline 117 & 5.737 & -6.045 & 0.358 & 63.549 & -6.045 & 0.358 & 63.549 & 242.720 & 318.569 \\
\hline 118 & 3.834 & -6.234 & -0.787 & 60.599 & -6.234 & -0.787 & 60.599 & 290.575 & 313.194 \\
\hline 119 & 7.850 & -3.992 & -0.215 & 75.774 & -3.992 & -0.215 & 75.774 & 300.280 & 310.242 \\
\hline 120 & 4.745 & -6.041 & 0.379 & 63.524 & -6.041 & 0.379 & 63.524 & 289.546 & 317.299 \\
\hline
\end{tabular}




\begin{tabular}{|c|c|c|c|c|c|c|c|c|c|}
\hline Vessel Number & Length & Starti & g Positic & $(\mathrm{cm})$ & Endin & Positio & $(\mathrm{cm})$ & Starting & Ending \\
\hline & $(\mathrm{cm})$ & $x$ & $y$ & $z$ & $x$ & $y$ & $z$ & Diameter $(\mu m)$ & Diameter $(\mu m)$ \\
\hline 121 & 2.248 & -5.501 & 1.443 & 63.961 & -5.501 & 1.443 & 63.961 & 440.326 & 301.434 \\
\hline 122 & 2.098 & -5.797 & 0.540 & 65.055 & -5.797 & 0.540 & 65.055 & 362.258 & 319.279 \\
\hline 123 & 3.651 & -1.909 & 1.571 & 78.699 & -1.909 & 1.571 & 78.699 & 176.853 & 197.846 \\
\hline 124 & 18.024 & -2.858 & -0.309 & 84.487 & -2.858 & -0.309 & 84.487 & 196.163 & 384.540 \\
\hline 125 & 2.619 & -3.668 & -0.361 & 77.399 & -3.668 & -0.361 & 77.399 & 257.722 & 185.330 \\
\hline 126 & 8.697 & -2.888 & -0.249 & 82.500 & -2.888 & -0.249 & 82.500 & 187.467 & 281.368 \\
\hline 127 & 3.020 & -4.478 & 1.967 & 66.161 & -4.478 & 1.967 & 66.161 & 451.889 & 355.006 \\
\hline 128 & 5.522 & -4.037 & 1.099 & 72.349 & -4.037 & 1.099 & 72.349 & 302.590 & 348.321 \\
\hline 129 & 2.774 & -4.077 & 0.457 & 74.899 & -4.077 & 0.457 & 74.899 & 311.434 & 183.606 \\
\hline 130 & 2.929 & -4.831 & 1.209 & 68.099 & -4.831 & 1.209 & 68.099 & 353.390 & 148.695 \\
\hline 131 & 4.181 & -3.785 & 2.138 & 67.636 & -3.785 & 2.138 & 67.636 & 256.606 & 148.695 \\
\hline 132 & 1.185 & -3.371 & 2.228 & 68.149 & -3.371 & 2.228 & 68.149 & 270.007 & 278.570 \\
\hline 133 & 4.653 & -3.453 & 0.538 & 78.074 & -3.453 & 0.538 & 78.074 & 268.080 & 262.257 \\
\hline 134 & 2.907 & -3.389 & 0.870 & 77.161 & -3.389 & 0.870 & 77.161 & 240.151 & 288.384 \\
\hline 135 & 1.350 & -3.453 & 0.536 & 78.036 & -3.453 & 0.536 & 78.036 & 324.187 & 438.282 \\
\hline 136 & 1.676 & -2.856 & 1.484 & 75.767 & -2.856 & 1.484 & 75.767 & 342.128 & 202.627 \\
\hline 137 & 1.322 & -3.188 & 1.225 & 75.899 & -3.188 & 1.225 & 75.899 & 129.173 & 148.695 \\
\hline 138 & 1.292 & -3.055 & 1.236 & 76.549 & -3.055 & 1.236 & 76.549 & 195.631 & 148.695 \\
\hline 139 & 3.687 & -2.608 & 1.020 & 80.549 & -2.608 & 1.020 & 80.549 & 402.900 & 256.086 \\
\hline 140 & 1.070 & -3.062 & 1.015 & 78.249 & -3.062 & 1.015 & 78.249 & 405.966 & 295.561 \\
\hline 141 & 3.684 & -2.831 & 0.704 & 80.999 & -2.831 & 0.704 & 80.999 & 192.838 & 148.911 \\
\hline 142 & 7.193 & 0.298 & 1.591 & 84.625 & 0.298 & 1.591 & 84.625 & 426.074 & 336.459 \\
\hline 143 & 4.620 & -2.867 & 0.378 & 83.424 & -2.867 & 0.378 & 83.424 & 413.357 & 312.904 \\
\hline 144 & 1.634 & -2.109 & 1.211 & 81.686 & -2.109 & 1.211 & 81.686 & 268.083 & 363.689 \\
\hline 145 & 5.496 & -2.159 & -1.130 & 82.500 & -2.159 & -1.130 & 82.500 & 336.281 & 387.105 \\
\hline 146 & 3.964 & -1.609 & 1.375 & 85.299 & -1.609 & 1.375 & 85.299 & 388.891 & 403.521 \\
\hline 147 & 0.611 & -5.796 & 0.547 & 65.055 & -5.796 & 0.547 & 65.055 & 129.173 & 148.695 \\
\hline 148 & 3.042 & -5.427 & 0.210 & 67.299 & -5.427 & 0.210 & 67.299 & 250.732 & 250.807 \\
\hline 149 & 2.541 & -3.668 & 1.876 & 69.099 & -3.668 & 1.876 & 69.099 & 143.525 & 165.217 \\
\hline 150 & 1.056 & -2.253 & 1.079 & 82.249 & -2.253 & 1.079 & 82.249 & 129.173 & 151.514 \\
\hline 151 & 1.644 & -1.762 & 1.335 & 83.024 & -1.762 & 1.335 & 83.024 & 303.139 & 424.521 \\
\hline 152 & 2.319 & -5.771 & 1.462 & 61.449 & -5.771 & 1.462 & 61.449 & 374.851 & 370.618 \\
\hline 153 & 3.649 & -5.083 & 1.979 & 63.449 & -5.083 & 1.979 & 63.449 & 308.184 & 236.507 \\
\hline 154 & 1.887 & -2.487 & 3.193 & 50.155 & -2.487 & 3.193 & 50.155 & 242.983 & 249.030 \\
\hline 155 & 1.085 & -2.581 & 3.182 & 50.202 & -2.581 & 3.182 & 50.202 & 412.429 & 351.186 \\
\hline 156 & 10.856 & -4.175 & 2.616 & 63.149 & -4.175 & 2.616 & 63.149 & 270.260 & 412.347 \\
\hline 157 & 8.458 & -4.313 & 3.167 & 56.599 & -4.313 & 3.167 & 56.599 & 320.374 & 368.794 \\
\hline 158 & 3.753 & -5.064 & 2.384 & 60.049 & -5.064 & 2.384 & 60.049 & 243.788 & 452.507 \\
\hline 159 & 2.502 & -6.220 & -0.333 & 51.486 & -6.220 & -0.333 & 51.486 & 272.919 & 517.289 \\
\hline 160 & 2.816 & -5.709 & -1.336 & 50.849 & -5.709 & -1.336 & 50.849 & 232.648 & 276.051 \\
\hline
\end{tabular}




\begin{tabular}{|c|c|c|c|c|c|c|c|c|c|}
\hline \multirow[t]{2}{*}{ Vessel Number } & \multirow{2}{*}{$\frac{\text { Length }}{(\mathrm{cm})}$} & \multicolumn{3}{|c|}{ Starting Position $(\mathrm{cm})$} & \multicolumn{3}{|c|}{ Ending Position $(\mathrm{cm})$} & \multirow{2}{*}{$\frac{\text { Starting }}{\text { Diameter }(\mu m)}$} & \multirow{2}{*}{$\frac{\text { Ending }}{\text { Diameter }(\mu m)}$} \\
\hline & & $x$ & $y$ & $z$ & $x$ & $y$ & $z$ & & \\
\hline 161 & 3.044 & -5.850 & -1.530 & 52.274 & -5.850 & -1.530 & 52.274 & 373.730 & 318.701 \\
\hline 162 & 3.032 & -1.150 & 3.521 & 50.574 & -1.150 & 3.521 & 50.574 & 287.460 & 330.905 \\
\hline 163 & 1.674 & -5.789 & -0.338 & 65.349 & -5.789 & -0.338 & 65.349 & 291.346 & 244.024 \\
\hline 164 & 1.672 & -5.928 & -0.460 & 64.449 & -5.928 & -0.460 & 64.449 & 252.688 & 174.527 \\
\hline 165 & 4.463 & -4.935 & -0.696 & 69.249 & -4.935 & -0.696 & 69.249 & 224.781 & 148.695 \\
\hline 166 & 3.654 & -5.237 & 2.020 & 51.499 & -5.237 & 2.020 & 51.499 & 339.795 & 388.130 \\
\hline 167 & 2.825 & -5.593 & 1.470 & 50.799 & -5.593 & 1.470 & 50.799 & 329.545 & 332.713 \\
\hline 168 & 2.945 & -6.313 & -0.803 & 52.899 & -6.313 & -0.803 & 52.899 & 172.988 & 193.187 \\
\hline 169 & 1.466 & -6.312 & -0.792 & 52.899 & -6.312 & -0.792 & 52.899 & 319.741 & 253.780 \\
\hline 170 & 6.526 & -6.441 & 0.691 & 58.949 & -6.441 & 0.691 & 58.949 & 401.758 & 387.799 \\
\hline 171 & 2.758 & -6.206 & 0.807 & 52.149 & -6.206 & 0.807 & 52.149 & 209.778 & 338.077 \\
\hline 172 & 2.726 & -6.209 & 0.798 & 52.149 & -6.209 & 0.798 & 52.149 & 393.410 & 442.575 \\
\hline 173 & 3.327 & -6.301 & 1.021 & 53.999 & -6.301 & 1.021 & 53.999 & 399.120 & 563.800 \\
\hline 174 & 2.200 & -6.302 & 1.018 & 53.999 & -6.302 & 1.018 & 53.999 & 341.240 & 245.508 \\
\hline 175 & 6.314 & -6.445 & 0.677 & 58.949 & -6.445 & 0.677 & 58.949 & 299.674 & 204.478 \\
\hline 176 & 6.441 & -3.493 & 3.536 & 55.949 & -3.493 & 3.536 & 55.949 & 335.329 & 366.200 \\
\hline 177 & 3.318 & -6.019 & 1.109 & 51.649 & -6.019 & 1.109 & 51.649 & 208.507 & 288.024 \\
\hline 178 & 2.653 & -6.197 & 0.831 & 52.149 & -6.197 & 0.831 & 52.149 & 364.958 & 420.116 \\
\hline 179 & 4.250 & -5.151 & 2.614 & 56.949 & -5.151 & 2.614 & 56.949 & 294.698 & 488.451 \\
\hline 180 & 2.293 & -5.224 & 2.555 & 57.099 & -5.224 & 2.555 & 57.099 & 263.177 & 302.952 \\
\hline 181 & 15.744 & 0.384 & 3.066 & 47.201 & 0.384 & 3.066 & 47.201 & 414.695 & 330.997 \\
\hline 182 & 2.027 & 4.362 & 2.915 & 26.517 & 4.362 & 2.915 & 26.517 & 469.959 & 487.953 \\
\hline 183 & 4.329 & 1.033 & 4.244 & 25.804 & 1.033 & 4.244 & 25.804 & 306.514 & 152.881 \\
\hline 184 & 3.929 & -0.281 & 3.774 & 27.976 & -0.281 & 3.774 & 27.976 & 361.314 & 399.468 \\
\hline 185 & 5.733 & 1.138 & 3.411 & 29.826 & 1.138 & 3.411 & 29.826 & 385.032 & 390.958 \\
\hline 186 & 5.829 & 0.748 & 3.396 & 32.476 & 0.748 & 3.396 & 32.476 & 348.180 & 298.337 \\
\hline 187 & 3.680 & 5.189 & 1.814 & 28.917 & 5.189 & 1.814 & 28.917 & 356.270 & 289.995 \\
\hline 188 & 7.634 & 0.686 & 3.321 & 40.250 & 0.686 & 3.321 & 40.250 & 329.387 & 351.215 \\
\hline 189 & 5.118 & 1.293 & 3.339 & 34.026 & 1.293 & 3.339 & 34.026 & 511.587 & 367.518 \\
\hline 190 & 7.293 & 4.598 & 2.356 & 36.779 & 4.598 & 2.356 & 36.779 & 357.168 & 445.953 \\
\hline 191 & 3.143 & 3.564 & 2.728 & 39.817 & 3.564 & 2.728 & 39.817 & 454.906 & 390.228 \\
\hline 192 & 1.480 & 3.915 & 2.717 & 37.548 & 3.915 & 2.717 & 37.548 & 159.215 & 158.312 \\
\hline 193 & 1.991 & 3.616 & 2.790 & 38.854 & 3.616 & 2.790 & 38.854 & 135.166 & 201.386 \\
\hline 194 & 2.563 & 4.169 & 2.605 & 36.004 & 4.169 & 2.605 & 36.004 & 129.743 & 148.695 \\
\hline 195 & 1.277 & 3.344 & 2.940 & 37.954 & 3.344 & 2.940 & 37.954 & 259.252 & 148.695 \\
\hline 196 & 2.763 & 3.162 & 2.648 & 42.317 & 3.162 & 2.648 & 42.317 & 298.505 & 241.441 \\
\hline 197 & 1.432 & 2.630 & 2.753 & 43.154 & 2.630 & 2.753 & 43.154 & 187.416 & 190.198 \\
\hline 198 & 2.596 & 2.667 & 2.674 & 43.854 & 2.667 & 2.674 & 43.854 & 309.108 & 244.924 \\
\hline 199 & 4.215 & 0.528 & 3.028 & 43.751 & 0.528 & 3.028 & 43.751 & 318.095 & 359.761 \\
\hline 200 & 4.064 & 5.612 & 1.270 & 31.392 & 5.612 & 1.270 & 31.392 & 369.913 & 371.276 \\
\hline
\end{tabular}




\begin{tabular}{|c|c|c|c|c|c|c|c|c|c|}
\hline Vessel Number & Length & Startin & Positi & $\mathrm{n}(\mathrm{cm})$ & Endin & Positic & $(\mathrm{cm})$ & Starting & Ending \\
\hline & $(\mathrm{cm})$ & $x$ & $y$ & $z$ & $x$ & $y$ & $z$ & Diameter $(\mu m)$ & Diameter $(\mu m)$ \\
\hline 201 & 7.744 & 5.813 & 0.963 & 40.067 & 5.813 & 0.963 & 40.067 & 380.095 & 318.657 \\
\hline 202 & 3.991 & 5.652 & 0.668 & 44.042 & 5.652 & 0.668 & 44.042 & 277.803 & 328.989 \\
\hline 203 & 5.507 & 5.236 & 1.879 & 36.567 & 5.236 & 1.879 & 36.567 & 278.912 & 499.453 \\
\hline 204 & 2.573 & 5.278 & 1.672 & 39.879 & 5.278 & 1.672 & 39.879 & 445.619 & 479.717 \\
\hline 205 & 2.014 & 4.901 & 2.089 & 38.617 & 4.901 & 2.089 & 38.617 & 356.633 & 303.697 \\
\hline 206 & 7.910 & 3.175 & 2.505 & 46.454 & 3.175 & 2.505 & 46.454 & 457.127 & 312.936 \\
\hline 207 & 2.977 & 4.611 & 2.107 & 41.104 & 4.611 & 2.107 & 41.104 & 282.481 & 169.279 \\
\hline 208 & 10.516 & 0.438 & 3.505 & 49.704 & 0.438 & 3.505 & 49.704 & 583.544 & 323.552 \\
\hline 209 & 2.780 & 5.089 & 1.591 & 42.548 & 5.089 & 1.591 & 42.548 & 335.529 & 288.357 \\
\hline 210 & 5.937 & 6.133 & 0.244 & 33.454 & 6.133 & 0.244 & 33.454 & 363.276 & 404.650 \\
\hline 211 & 2.382 & 6.072 & 0.118 & 28.154 & 6.072 & 0.118 & 28.154 & 398.337 & 346.356 \\
\hline 212 & 6.266 & 6.037 & 0.696 & 34.054 & 6.037 & 0.696 & 34.054 & 401.366 & 375.686 \\
\hline 213 & 3.174 & 5.931 & 1.008 & 36.554 & 5.931 & 1.008 & 36.554 & 163.490 & 185.015 \\
\hline 214 & 4.500 & 6.050 & 0.436 & 39.254 & 6.050 & 0.436 & 39.254 & 326.310 & 341.816 \\
\hline 215 & 4.161 & 5.921 & 0.333 & 42.154 & 5.921 & 0.333 & 42.154 & 261.275 & 151.422 \\
\hline 216 & 1.496 & 6.037 & 0.266 & 40.354 & 6.037 & 0.266 & 40.354 & 216.328 & 163.492 \\
\hline 217 & 4.165 & 5.202 & 1.274 & 44.467 & 5.202 & 1.274 & 44.467 & 389.023 & 206.065 \\
\hline 218 & 2.360 & 4.969 & 1.453 & 45.804 & 4.969 & 1.453 & 45.804 & 226.470 & 151.130 \\
\hline 219 & 2.912 & 5.188 & 1.234 & 46.554 & 5.188 & 1.234 & 46.554 & 187.884 & 179.610 \\
\hline 220 & 2.703 & 5.558 & 0.598 & 46.455 & 5.558 & 0.598 & 46.455 & 427.488 & 461.026 \\
\hline 221 & 2.977 & 5.541 & 0.696 & 47.042 & 5.541 & 0.696 & 47.042 & 320.067 & 349.587 \\
\hline 222 & 2.012 & 5.172 & 1.423 & 48.104 & 5.172 & 1.423 & 48.104 & 132.348 & 148.695 \\
\hline 223 & 1.943 & 5.624 & 0.883 & 48.929 & 5.624 & 0.883 & 48.929 & 362.920 & 352.401 \\
\hline 224 & 4.974 & 3.821 & 2.215 & 45.504 & 3.821 & 2.215 & 45.504 & 264.342 & 148.695 \\
\hline 225 & 4.498 & 4.410 & 1.997 & 47.554 & 4.410 & 1.997 & 47.554 & 314.327 & 315.987 \\
\hline 226 & 7.572 & 3.358 & 3.044 & 50.554 & 3.358 & 3.044 & 50.554 & 169.887 & 260.914 \\
\hline 227 & 4.376 & 2.116 & 3.288 & 49.754 & 2.116 & 3.288 & 49.754 & 181.785 & 205.074 \\
\hline 228 & 5.886 & 1.845 & 3.630 & 51.751 & 1.845 & 3.630 & 51.751 & 323.181 & 348.095 \\
\hline 229 & 3.370 & 5.468 & 2.000 & 59.967 & 5.468 & 2.000 & 59.967 & 330.271 & 292.333 \\
\hline 230 & 6.627 & 2.236 & 3.064 & 65.154 & 2.236 & 3.064 & 65.154 & 246.471 & 317.210 \\
\hline 231 & 9.920 & 1.407 & 2.972 & 66.651 & 1.407 & 2.972 & 66.651 & 229.095 & 235.878 \\
\hline 232 & 6.320 & 3.777 & 1.905 & 68.517 & 3.777 & 1.905 & 68.517 & 361.866 & 372.579 \\
\hline 233 & 4.676 & 4.276 & 2.088 & 66.104 & 4.276 & 2.088 & 66.104 & 400.632 & 268.652 \\
\hline 234 & 3.557 & 4.741 & 2.312 & 62.404 & 4.741 & 2.312 & 62.404 & 284.636 & 235.242 \\
\hline 235 & 5.184 & 0.134 & 2.552 & 70.004 & 0.134 & 2.552 & 70.004 & 307.597 & 324.792 \\
\hline 236 & 7.370 & -0.264 & 2.103 & 75.004 & -0.264 & 2.103 & 75.004 & 312.302 & 477.914 \\
\hline 237 & 8.847 & 0.020 & 1.840 & 79.054 & 0.020 & 1.840 & 79.054 & 252.410 & 380.125 \\
\hline 238 & 7.723 & 3.045 & 3.215 & 61.617 & 3.045 & 3.215 & 61.617 & 329.460 & 325.207 \\
\hline 239 & 2.100 & 1.906 & 3.440 & 62.454 & 1.906 & 3.440 & 62.454 & 298.345 & 332.700 \\
\hline 240 & 3.990 & 1.726 & 3.264 & 64.454 & 1.726 & 3.264 & 64.454 & 268.169 & 155.817 \\
\hline
\end{tabular}




\begin{tabular}{|c|c|c|c|c|c|c|c|c|c|}
\hline \multirow[t]{2}{*}{ Vessel Number } & \multirow{2}{*}{$\frac{\text { Length }}{(\mathrm{cm})}$} & \multicolumn{3}{|c|}{ Starting Position $(\mathrm{cm})$} & \multicolumn{3}{|c|}{ Ending Position $(\mathrm{cm})$} & \multirow{2}{*}{$\begin{array}{c}\text { Starting } \\
\text { Diameter }(\mu m)\end{array}$} & \multirow{2}{*}{$\begin{array}{c}\text { Ending } \\
\text { Diameter }(\mu m)\end{array}$} \\
\hline & & $x$ & $y$ & $z$ & $x$ & $y$ & $z$ & & \\
\hline 241 & 0.949 & 5.439 & 0.901 & 66.067 & 5.439 & 0.901 & 66.067 & 363.872 & 264.914 \\
\hline 242 & 10.257 & 1.432 & 2.100 & 73.554 & 1.432 & 2.100 & 73.554 & 339.270 & 342.531 \\
\hline 243 & 2.847 & 4.763 & 1.428 & 67.404 & 4.763 & 1.428 & 67.404 & 290.639 & 180.168 \\
\hline 244 & 6.442 & 1.694 & 1.776 & 76.892 & 1.694 & 1.776 & 76.892 & 255.316 & 251.169 \\
\hline 245 & 3.046 & 0.633 & 1.752 & 79.854 & 0.633 & 1.752 & 79.854 & 394.136 & 356.678 \\
\hline 246 & 5.544 & 0.756 & 1.737 & 79.754 & 0.756 & 1.737 & 79.754 & 382.691 & 361.550 \\
\hline 247 & 1.871 & 1.711 & 1.640 & 78.154 & 1.711 & 1.640 & 78.154 & 220.145 & 214.897 \\
\hline 248 & 1.629 & 0.361 & 1.747 & 81.254 & 0.361 & 1.747 & 81.254 & 298.446 & 330.161 \\
\hline 249 & 2.004 & 4.437 & 2.306 & 49.529 & 4.437 & 2.306 & 49.529 & 378.759 & 295.298 \\
\hline 250 & 2.100 & 3.869 & 2.993 & 51.542 & 3.869 & 2.993 & 51.542 & 309.703 & 357.563 \\
\hline 251 & 6.667 & 2.293 & 3.915 & 56.401 & 2.293 & 3.915 & 56.401 & 248.596 & 266.743 \\
\hline 252 & 4.881 & 1.591 & 3.987 & 55.426 & 1.591 & 3.987 & 55.426 & 200.057 & 275.085 \\
\hline 253 & 10.408 & 2.535 & 3.707 & 58.701 & 2.535 & 3.707 & 58.701 & 315.139 & 345.294 \\
\hline 254 & 3.729 & -5.437 & -2.523 & 25.105 & -5.437 & -2.523 & 25.105 & 363.305 & 284.877 \\
\hline 255 & 3.571 & -3.982 & -3.134 & 26.418 & -3.982 & -3.134 & 26.418 & 350.672 & 403.671 \\
\hline 256 & 6.929 & -2.635 & -3.151 & 29.955 & -2.635 & -3.151 & 29.955 & 275.811 & 363.071 \\
\hline 257 & 5.231 & -2.910 & -3.134 & 29.205 & -2.910 & -3.134 & 29.205 & 288.963 & 210.702 \\
\hline 258 & 4.488 & -3.888 & -2.699 & 34.141 & -3.888 & -2.699 & 34.141 & 269.980 & 148.695 \\
\hline 259 & 5.924 & -3.054 & -3.058 & 35.505 & -3.054 & -3.058 & 35.505 & 248.657 & 385.360 \\
\hline 260 & 2.496 & -3.944 & -2.716 & 36.805 & -3.944 & -2.716 & 36.805 & 254.200 & 148.695 \\
\hline 261 & 5.142 & -3.914 & -2.603 & 39.827 & -3.914 & -2.603 & 39.827 & 273.685 & 189.320 \\
\hline 262 & 3.136 & -2.431 & -3.182 & 32.305 & -2.431 & -3.182 & 32.305 & 174.585 & 165.725 \\
\hline 263 & 7.763 & -2.099 & -3.308 & 35.555 & -2.099 & -3.308 & 35.555 & 293.352 & 311.333 \\
\hline 264 & 8.451 & -2.099 & -3.308 & 35.580 & -2.099 & -3.308 & 35.580 & 329.996 & 214.887 \\
\hline 265 & 5.800 & -3.697 & -2.433 & 43.702 & -3.697 & -2.433 & 43.702 & 283.681 & 303.199 \\
\hline 266 & 13.735 & -2.605 & -2.687 & 43.955 & -2.605 & -2.687 & 43.955 & 334.443 & 344.345 \\
\hline 267 & 4.071 & -4.086 & -2.463 & 49.271 & -4.086 & -2.463 & 49.271 & 331.036 & 345.002 \\
\hline 268 & 3.206 & -2.618 & -2.686 & 46.405 & -2.618 & -2.686 & 46.405 & 197.806 & 148.695 \\
\hline 269 & 5.615 & 1.279 & -3.394 & 31.855 & 1.279 & -3.394 & 31.855 & 238.292 & 312.757 \\
\hline 270 & 6.060 & -0.200 & -3.414 & 39.330 & -0.200 & -3.414 & 39.330 & 213.221 & 272.298 \\
\hline 271 & 1.111 & 1.774 & -3.319 & 32.218 & 1.774 & -3.319 & 32.218 & 188.197 & 173.913 \\
\hline 272 & 5.552 & 1.191 & -3.460 & 36.055 & 1.191 & -3.460 & 36.055 & 178.918 & 149.857 \\
\hline 273 & 3.551 & 2.789 & -3.133 & 29.855 & 2.789 & -3.133 & 29.855 & 235.584 & 202.133 \\
\hline 274 & 3.024 & -1.208 & -3.203 & 41.055 & -1.208 & -3.203 & 41.055 & 164.771 & 148.695 \\
\hline 275 & 1.290 & -1.804 & -3.073 & 41.455 & -1.804 & -3.073 & 41.455 & 158.861 & 173.487 \\
\hline 276 & 1.926 & -1.459 & -3.034 & 42.255 & -1.459 & -3.034 & 42.255 & 129.173 & 148.695 \\
\hline 277 & 6.692 & -2.410 & -2.958 & 48.218 & -2.410 & -2.958 & 48.218 & 258.339 & 340.071 \\
\hline 278 & 2.640 & -3.765 & -2.774 & 50.055 & -3.765 & -2.774 & 50.055 & 373.598 & 198.535 \\
\hline 279 & 1.186 & -4.118 & -2.512 & 49.548 & -4.118 & -2.512 & 49.548 & 241.956 & 379.806 \\
\hline 280 & 3.466 & -4.242 & -2.891 & 52.005 & -4.242 & -2.891 & 52.005 & 221.142 & 148.695 \\
\hline
\end{tabular}




\begin{tabular}{|c|c|c|c|c|c|c|c|c|c|}
\hline Vessel Number & Length & Starti & g Positic & $(\mathrm{cm})$ & Endin & Positic & $(\mathrm{cm})$ & Starting & Ending \\
\hline & $(\mathrm{cm})$ & $x$ & $y$ & $z$ & $x$ & $y$ & $z$ & Diameter $(\mu m)$ & Diameter $(\mu m)$ \\
\hline 281 & 5.753 & -4.773 & -2.780 & 53.705 & -4.773 & -2.780 & 53.705 & 284.326 & 263.876 \\
\hline 282 & 10.740 & 2.668 & -3.183 & 36.655 & 2.668 & -3.183 & 36.655 & 307.073 & 363.730 \\
\hline 283 & 13.424 & -3.891 & -3.293 & 53.955 & -3.891 & -3.293 & 53.955 & 380.971 & 290.578 \\
\hline 284 & 2.599 & -5.009 & -2.688 & 55.802 & -5.009 & -2.688 & 55.802 & 195.028 & 186.798 \\
\hline 285 & 2.489 & -5.019 & -2.696 & 55.605 & -5.019 & -2.696 & 55.605 & 290.001 & 250.373 \\
\hline 286 & 3.471 & 3.452 & -2.999 & 28.455 & 3.452 & -2.999 & 28.455 & 363.802 & 296.126 \\
\hline 287 & 4.210 & 3.441 & -3.004 & 28.455 & 3.441 & -3.004 & 28.455 & 430.710 & 443.920 \\
\hline 288 & 6.135 & 1.864 & -3.363 & 36.205 & 1.864 & -3.363 & 36.205 & 288.620 & 332.241 \\
\hline 289 & 4.450 & 4.419 & -2.438 & 29.030 & 4.419 & -2.438 & 29.030 & 205.869 & 255.239 \\
\hline 290 & 4.541 & 4.404 & -2.446 & 29.043 & 4.404 & -2.446 & 29.043 & 320.001 & 235.821 \\
\hline 291 & 2.247 & 3.868 & -2.667 & 31.555 & 3.868 & -2.667 & 31.555 & 314.112 & 471.450 \\
\hline 292 & 4.474 & 1.854 & -3.364 & 36.230 & 1.854 & -3.364 & 36.230 & 314.112 & 314.300 \\
\hline 293 & 12.784 & -3.944 & -3.355 & 55.918 & -3.944 & -3.355 & 55.918 & 262.212 & 381.802 \\
\hline 294 & 2.659 & -4.925 & -2.714 & 57.905 & -4.925 & -2.714 & 57.905 & 415.019 & 226.179 \\
\hline 295 & 4.009 & -4.582 & -2.791 & 58.905 & -4.582 & -2.791 & 58.905 & 316.288 & 151.180 \\
\hline 296 & 1.288 & 2.859 & -3.118 & 37.455 & 2.859 & -3.118 & 37.455 & 376.773 & 245.467 \\
\hline 297 & 3.102 & 2.425 & -3.133 & 39.355 & 2.425 & -3.133 & 39.355 & 129.173 & 192.215 \\
\hline 298 & 1.663 & 3.397 & -2.947 & 36.355 & 3.397 & -2.947 & 36.355 & 173.576 & 164.767 \\
\hline 299 & 2.352 & 3.945 & -2.675 & 33.355 & 3.945 & -2.675 & 33.355 & 170.548 & 148.695 \\
\hline 300 & 10.966 & 5.953 & -0.388 & 41.655 & 5.953 & -0.388 & 41.655 & 445.040 & 324.008 \\
\hline 301 & 10.985 & 2.598 & -2.807 & 42.518 & 2.598 & -2.807 & 42.518 & 222.948 & 229.548 \\
\hline 302 & 2.742 & 1.839 & -2.835 & 44.855 & 1.839 & -2.835 & 44.855 & 327.473 & 266.555 \\
\hline 303 & 4.950 & 1.486 & -3.244 & 48.818 & 1.486 & -3.244 & 48.818 & 257.975 & 296.964 \\
\hline 304 & 2.601 & 0.117 & -3.629 & 50.518 & 0.117 & -3.629 & 50.518 & 485.757 & 387.716 \\
\hline 305 & 7.298 & -3.811 & -3.270 & 58.718 & -3.811 & -3.270 & 58.718 & 280.171 & 322.515 \\
\hline 306 & 2.953 & -0.595 & -4.013 & 53.255 & -0.595 & -4.013 & 53.255 & 169.136 & 174.144 \\
\hline 307 & 2.420 & -4.499 & -2.694 & 60.405 & -4.499 & -2.694 & 60.405 & 477.462 & 361.430 \\
\hline 308 & 9.450 & -2.843 & -3.347 & 60.955 & -2.843 & -3.347 & 60.955 & 374.601 & 241.057 \\
\hline 309 & 2.281 & -3.737 & -2.986 & 61.305 & -3.737 & -2.986 & 61.305 & 302.569 & 273.227 \\
\hline 310 & 3.867 & -4.463 & -2.377 & 63.327 & -4.463 & -2.377 & 63.327 & 326.968 & 376.385 \\
\hline 311 & 5.472 & 5.656 & -1.214 & 31.730 & 5.656 & -1.214 & 31.730 & 386.442 & 220.315 \\
\hline 312 & 3.541 & 4.838 & -2.199 & 35.693 & 4.838 & -2.199 & 35.693 & 353.108 & 367.041 \\
\hline 313 & 2.603 & 4.130 & -2.545 & 39.018 & 4.130 & -2.545 & 39.018 & 318.740 & 309.501 \\
\hline 314 & 2.808 & 4.885 & -2.113 & 38.355 & 4.885 & -2.113 & 38.355 & 260.406 & 255.239 \\
\hline 315 & 8.775 & 1.607 & -3.701 & 51.718 & 1.607 & -3.701 & 51.718 & 279.553 & 366.913 \\
\hline 316 & 6.377 & 4.376 & -2.400 & 49.793 & 4.376 & -2.400 & 49.793 & 239.171 & 205.210 \\
\hline 317 & 4.482 & 4.251 & -3.019 & 53.055 & 4.251 & -3.019 & 53.055 & 431.923 & 148.695 \\
\hline 318 & 14.935 & 4.500 & 0.879 & 70.580 & 4.500 & 0.879 & 70.580 & 334.253 & 344.149 \\
\hline 319 & 3.063 & 1.674 & -3.956 & 53.955 & 1.674 & -3.956 & 53.955 & 148.426 & 148.695 \\
\hline 320 & 1.989 & 1.188 & -3.988 & 53.618 & 1.188 & -3.988 & 53.618 & 308.152 & 424.550 \\
\hline
\end{tabular}




\begin{tabular}{|c|c|c|c|c|c|c|c|c|c|}
\hline \multirow[t]{2}{*}{ Vessel Number } & \multirow{2}{*}{$\frac{\text { Length }}{(\mathrm{cm})}$} & \multicolumn{3}{|c|}{ Starting Position $(\mathrm{cm})$} & \multicolumn{3}{|c|}{ Ending Position $(\mathrm{cm})$} & \multirow{2}{*}{$\frac{\text { Starting }}{\text { Diameter }(\mu m)}$} & \multirow{2}{*}{$\begin{array}{c}\text { Ending } \\
\text { Diameter }(\mu m)\end{array}$} \\
\hline & & $x$ & $y$ & $z$ & $x$ & $y$ & $z$ & & \\
\hline 321 & 2.709 & -0.157 & -4.186 & 55.818 & -0.157 & -4.186 & 55.818 & 288.029 & 307.846 \\
\hline 322 & 7.374 & 2.463 & -3.103 & 64.343 & 2.463 & -3.103 & 64.343 & 289.127 & 315.745 \\
\hline 323 & 1.697 & 0.176 & -4.147 & 57.155 & 0.176 & -4.147 & 57.155 & 186.295 & 237.912 \\
\hline 324 & 2.718 & -0.869 & -4.043 & 58.168 & -0.869 & -4.043 & 58.168 & 284.045 & 286.775 \\
\hline 325 & 2.771 & -1.427 & -3.657 & 60.755 & -1.427 & -3.657 & 60.755 & 393.994 & 344.744 \\
\hline 326 & 3.405 & -2.381 & -3.124 & 64.518 & -2.381 & -3.124 & 64.518 & 386.015 & 381.032 \\
\hline 327 & 2.477 & -3.517 & -2.481 & 66.105 & -3.517 & -2.481 & 66.105 & 321.722 & 307.556 \\
\hline 328 & 6.787 & -2.407 & -2.276 & 69.755 & -2.407 & -2.276 & 69.755 & 268.235 & 371.101 \\
\hline 329 & 2.556 & -2.295 & -2.043 & 71.805 & -2.295 & -2.043 & 71.805 & 186.103 & 338.727 \\
\hline 330 & 2.904 & -2.388 & -2.773 & 66.605 & -2.388 & -2.773 & 66.605 & 184.677 & 148.695 \\
\hline 331 & 2.207 & -0.143 & -3.774 & 60.518 & -0.143 & -3.774 & 60.518 & 173.778 & 266.722 \\
\hline 332 & 5.110 & -0.382 & -2.985 & 66.930 & -0.382 & -2.985 & 66.930 & 321.220 & 269.045 \\
\hline 333 & 5.280 & -1.557 & -2.038 & 73.805 & -1.557 & -2.038 & 73.805 & 307.096 & 392.184 \\
\hline 334 & 2.721 & -1.790 & -1.819 & 76.202 & -1.790 & -1.819 & 76.202 & 399.608 & 335.281 \\
\hline 335 & 4.194 & -1.184 & -1.658 & 79.943 & -1.184 & -1.658 & 79.943 & 174.383 & 301.108 \\
\hline 336 & 2.077 & -1.837 & -1.398 & 81.105 & -1.837 & -1.398 & 81.105 & 314.112 & 361.586 \\
\hline 337 & 2.048 & -1.219 & -1.538 & 82.055 & -1.219 & -1.538 & 82.055 & 307.073 & 437.150 \\
\hline 338 & 5.007 & 1.784 & -2.093 & 72.830 & 1.784 & -2.093 & 72.830 & 470.750 & 383.179 \\
\hline 339 & 4.600 & 3.010 & -1.344 & 75.755 & 3.010 & -1.344 & 75.755 & 262.706 & 251.620 \\
\hline 340 & 4.697 & 2.488 & -1.302 & 78.280 & 2.488 & -1.302 & 78.280 & 331.812 & 393.716 \\
\hline 341 & 7.450 & 5.255 & -1.693 & 39.730 & 5.255 & -1.693 & 39.730 & 346.185 & 398.506 \\
\hline 342 & 3.780 & 3.928 & -2.204 & 44.255 & 3.928 & -2.204 & 44.255 & 379.868 & 383.520 \\
\hline 343 & 4.898 & 4.476 & -2.044 & 48.055 & 4.476 & -2.044 & 48.055 & 300.277 & 193.229 \\
\hline 344 & 3.095 & 3.209 & -2.591 & 47.355 & 3.209 & -2.591 & 47.355 & 334.421 & 362.948 \\
\hline 345 & 4.058 & 2.544 & -3.637 & 52.718 & 2.544 & -3.637 & 52.718 & 364.831 & 288.097 \\
\hline 346 & 17.519 & 5.489 & 0.719 & 66.155 & 5.489 & 0.719 & 66.155 & 340.288 & 175.182 \\
\hline 347 & 6.905 & 3.453 & -3.552 & 55.268 & 3.453 & -3.552 & 55.268 & 251.999 & 345.022 \\
\hline 348 & 4.648 & 4.107 & -2.698 & 62.368 & 4.107 & -2.698 & 62.368 & 383.481 & 353.151 \\
\hline 349 & 3.675 & 4.260 & -2.900 & 59.668 & 4.260 & -2.900 & 59.668 & 347.808 & 297.390 \\
\hline 350 & 3.484 & 4.755 & -2.340 & 61.955 & 4.755 & -2.340 & 61.955 & 250.896 & 148.695 \\
\hline 351 & 2.321 & 4.103 & -2.700 & 62.368 & 4.103 & -2.700 & 62.368 & 270.786 & 406.423 \\
\hline 352 & 4.674 & 1.080 & -3.773 & 60.055 & 1.080 & -3.773 & 60.055 & 227.667 & 314.975 \\
\hline 353 & 3.231 & 1.698 & -3.400 & 63.355 & 1.698 & -3.400 & 63.355 & 143.517 & 221.649 \\
\hline 354 & 7.822 & 0.086 & -2.322 & 72.418 & 0.086 & -2.322 & 72.418 & 292.855 & 357.566 \\
\hline 355 & 5.452 & -0.243 & -1.992 & 77.155 & -0.243 & -1.992 & 77.155 & 240.988 & 148.695 \\
\hline 356 & 4.320 & 0.299 & -1.785 & 79.155 & 0.299 & -1.785 & 79.155 & 320.195 & 204.456 \\
\hline 357 & 3.271 & 1.047 & -3.422 & 63.830 & 1.047 & -3.422 & 63.830 & 409.677 & 368.253 \\
\hline 358 & 9.843 & 1.492 & -1.765 & 77.818 & 1.492 & -1.765 & 77.818 & 271.480 & 279.517 \\
\hline 359 & 4.448 & 1.193 & -2.751 & 67.955 & 1.193 & -2.751 & 67.955 & 281.758 & 148.695 \\
\hline 360 & 6.756 & 4.341 & -0.880 & 71.330 & 4.341 & -0.880 & 71.330 & 372.815 & 743.476 \\
\hline
\end{tabular}




\begin{tabular}{|c|c|c|c|c|c|c|c|c|c|}
\hline Vessel Number & Length & Starti & g Positi & $\mathrm{n}(\mathrm{cm})$ & Endir & Positi & $(\mathrm{cm})$ & Starting & Ending \\
\hline & $(\mathrm{cm})$ & $x$ & $y$ & $z$ & $x$ & $y$ & $z$ & Diameter $(\mu m)$ & Diameter $(\mu m)$ \\
\hline 361 & 11.039 & 4.754 & -0.573 & 70.118 & 4.754 & -0.573 & 70.118 & 272.644 & 369.876 \\
\hline 362 & 1.643 & 4.492 & -2.343 & 63.630 & 4.492 & -2.343 & 63.630 & 281.556 & 366.688 \\
\hline 363 & 3.118 & 2.735 & -2.482 & 67.530 & 2.735 & -2.482 & 67.530 & 199.814 & 306.684 \\
\hline 364 & 7.298 & 3.877 & -0.721 & 74.718 & 3.877 & -0.721 & 74.718 & 278.013 & 253.006 \\
\hline 365 & 2.877 & 2.228 & -2.274 & 70.055 & 2.228 & -2.274 & 70.055 & 187.245 & 152.413 \\
\hline 366 & 6.029 & 3.559 & -0.655 & 76.730 & 3.559 & -0.655 & 76.730 & 287.006 & 348.253 \\
\hline 367 & 4.084 & 5.265 & -0.985 & 66.455 & 5.265 & -0.985 & 66.455 & 274.017 & 223.043 \\
\hline 368 & 6.761 & 4.196 & 0.925 & 72.030 & 4.196 & 0.925 & 72.030 & 281.468 & 204.921 \\
\hline 369 & 3.076 & 4.494 & -0.100 & 72.355 & 4.494 & -0.100 & 72.355 & 134.824 & 155.201 \\
\hline 370 & 4.210 & 3.716 & 1.004 & 74.080 & 3.716 & 1.004 & 74.080 & 235.514 & 428.660 \\
\hline 371 & 2.070 & 3.698 & 1.021 & 74.118 & 3.698 & 1.021 & 74.118 & 215.090 & 234.892 \\
\hline 372 & 5.287 & 0.063 & 1.794 & 80.055 & 0.063 & 1.794 & 80.055 & 260.364 & 315.926 \\
\hline 373 & 4.107 & 3.968 & 0.311 & 75.355 & 3.968 & 0.311 & 75.355 & 228.272 & 195.859 \\
\hline 374 & 4.395 & 3.023 & 0.917 & 78.030 & 3.023 & 0.917 & 78.030 & 272.166 & 260.681 \\
\hline 375 & 3.462 & 3.025 & 0.914 & 78.018 & 3.025 & 0.914 & 78.018 & 336.678 & 173.323 \\
\hline 376 & 8.039 & 0.060 & 1.772 & 80.355 & 0.060 & 1.772 & 80.355 & 196.585 & 357.805 \\
\hline 377 & 7.650 & 6.153 & 0.374 & 36.455 & 6.153 & 0.374 & 36.455 & 367.405 & 331.776 \\
\hline 378 & 1.988 & 5.985 & 0.828 & 37.655 & 5.985 & 0.828 & 37.655 & 508.605 & 231.315 \\
\hline 379 & 4.360 & 5.795 & 0.830 & 41.455 & 5.795 & 0.830 & 41.455 & 265.520 & 299.714 \\
\hline 380 & 3.270 & 5.985 & -0.461 & 28.655 & 5.985 & -0.461 & 28.655 & 365.110 & 545.273 \\
\hline 381 & 10.456 & 5.539 & 0.704 & 45.555 & 5.539 & 0.704 & 45.555 & 297.299 & 228.154 \\
\hline 382 & 1.886 & 5.916 & -0.555 & 30.430 & 5.916 & -0.555 & 30.430 & 444.629 & 397.767 \\
\hline 383 & 16.429 & 5.705 & -2.036 & 54.993 & 5.705 & -2.036 & 54.993 & 268.033 & 308.543 \\
\hline 384 & 6.659 & 5.487 & -1.251 & 41.893 & 5.487 & -1.251 & 41.893 & 339.386 & 349.434 \\
\hline 385 & 3.978 & 5.239 & -1.164 & 45.855 & 5.239 & -1.164 & 45.855 & 304.810 & 306.834 \\
\hline 386 & 2.995 & 5.664 & 0.287 & 46.055 & 5.664 & 0.287 & 46.055 & 368.748 & 303.199 \\
\hline 387 & 2.206 & 5.873 & -0.011 & 43.155 & 5.873 & -0.011 & 43.155 & 425.653 & 296.824 \\
\hline 388 & 1.769 & 5.830 & -0.248 & 43.255 & 5.830 & -0.248 & 43.255 & 370.564 & 503.833 \\
\hline 389 & 1.975 & 5.623 & 0.490 & 45.555 & 5.623 & 0.490 & 45.555 & 316.644 & 270.744 \\
\hline 390 & 10.062 & 6.444 & 0.475 & 58.855 & 6.444 & 0.475 & 58.855 & 229.717 & 426.389 \\
\hline 391 & 7.439 & 6.450 & 0.684 & 54.405 & 6.450 & 0.684 & 54.405 & 369.546 & 284.818 \\
\hline 392 & 3.603 & 6.289 & -0.521 & 51.593 & 6.289 & -0.521 & 51.593 & 406.894 & 334.564 \\
\hline 393 & 4.517 & 6.552 & 0.467 & 56.655 & 6.552 & 0.467 & 56.655 & 194.028 & 299.659 \\
\hline 394 & 4.761 & 6.529 & -0.581 & 56.155 & 6.529 & -0.581 & 56.155 & 312.840 & 259.687 \\
\hline 395 & 3.612 & 5.721 & -2.067 & 57.555 & 5.721 & -2.067 & 57.555 & 226.052 & 205.719 \\
\hline 396 & 3.499 & 6.246 & -1.042 & 58.955 & 6.246 & -1.042 & 58.955 & 351.655 & 286.238 \\
\hline 397 & 3.223 & 6.225 & 0.603 & 60.905 & 6.225 & 0.603 & 60.905 & 434.343 & 197.637 \\
\hline 398 & 1.775 & 6.227 & -0.667 & 60.518 & 6.227 & -0.667 & 60.518 & 295.637 & 322.235 \\
\hline 399 & 2.642 & 6.048 & 0.593 & 62.505 & 6.048 & 0.593 & 62.505 & 294.550 & 259.604 \\
\hline 400 & 1.965 & 6.159 & -0.140 & 62.355 & 6.159 & -0.140 & 62.355 & 349.894 & 209.393 \\
\hline
\end{tabular}




\begin{tabular}{|c|c|c|c|c|c|c|c|c|c|}
\hline \multirow[t]{2}{*}{ Vessel Number } & \multirow{2}{*}{$\frac{\text { Length }}{(\mathrm{cm})}$} & \multicolumn{3}{|c|}{ Starting Position $(\mathrm{cm})$} & \multicolumn{3}{|c|}{ Ending Position $(\mathrm{cm})$} & \multirow{2}{*}{$\begin{array}{c}\text { Starting } \\
\text { Diameter }(\mu m)\end{array}$} & \multirow{2}{*}{$\begin{array}{c}\text { Ending } \\
\text { Diameter }(\mu m)\end{array}$} \\
\hline & & $x$ & $y$ & $z$ & $x$ & $y$ & $z$ & & \\
\hline 401 & 1.543 & 3.024 & -1.048 & 77.768 & 3.024 & -1.048 & 77.768 & 358.677 & 583.909 \\
\hline 402 & 1.840 & 3.449 & -0.012 & 78.349 & 3.449 & -0.012 & 78.349 & 312.307 & 409.902 \\
\hline 403 & 2.646 & -0.672 & -1.676 & 81.280 & -0.672 & -1.676 & 81.280 & 520.917 & 305.761 \\
\hline 404 & 2.984 & 1.598 & 1.547 & 80.055 & 1.598 & 1.547 & 80.055 & 224.664 & 417.010 \\
\hline 405 & 3.631 & -0.093 & 1.759 & 81.055 & -0.093 & 1.759 & 81.055 & 385.500 & 251.030 \\
\hline 406 & 1.375 & 2.508 & -1.288 & 78.343 & 2.508 & -1.288 & 78.343 & 421.309 & 338.727 \\
\hline 407 & 3.622 & 3.138 & -0.178 & 79.955 & 3.138 & -0.178 & 79.955 & 426.029 & 303.248 \\
\hline 408 & 3.146 & 0.708 & -1.710 & 80.968 & 0.708 & -1.710 & 80.968 & 453.097 & 282.864 \\
\hline 409 & 1.738 & 2.797 & -0.825 & 79.730 & 2.797 & -0.825 & 79.730 & 376.067 & 359.508 \\
\hline 410 & 1.963 & 0.710 & -1.710 & 80.955 & 0.710 & -1.710 & 80.955 & 645.863 & 534.076 \\
\hline 411 & 2.051 & 1.454 & -1.587 & 79.955 & 1.454 & -1.587 & 79.955 & 217.979 & 264.496 \\
\hline 412 & 3.098 & 2.083 & -1.343 & 79.855 & 2.083 & -1.343 & 79.855 & 391.284 & 418.205 \\
\hline 413 & 2.334 & 3.152 & 0.054 & 80.055 & 3.152 & 0.054 & 80.055 & 516.679 & 412.397 \\
\hline 414 & 1.415 & -1.216 & -1.539 & 82.055 & -1.216 & -1.539 & 82.055 & 404.845 & 466.031 \\
\hline 415 & 1.573 & -2.055 & -1.175 & 82.978 & -2.055 & -1.175 & 82.978 & 206.083 & 302.410 \\
\hline 416 & 4.058 & 1.180 & 1.578 & 81.005 & 1.180 & 1.578 & 81.005 & 283.082 & 271.136 \\
\hline 417 & 2.364 & -1.730 & 1.348 & 82.255 & -1.730 & 1.348 & 82.255 & 344.124 & 237.680 \\
\hline 418 & 1.184 & 2.543 & -1.005 & 80.355 & 2.543 & -1.005 & 80.355 & 338.069 & 348.078 \\
\hline 419 & 1.330 & -0.844 & -1.602 & 82.500 & -0.844 & -1.602 & 82.500 & 263.433 & 168.211 \\
\hline 420 & 1.719 & 1.457 & -1.451 & 82.255 & 1.457 & -1.451 & 82.255 & 322.236 & 668.716 \\
\hline 421 & 2.378 & -0.237 & -1.677 & 82.500 & -0.237 & -1.677 & 82.500 & 234.876 & 191.183 \\
\hline
\end{tabular}




\begin{tabular}{|c|c|c|c|c|c|}
\hline $\begin{array}{l}\text { Parent } \\
\text { Index }\end{array}$ & $\begin{array}{l}\text { Daughter } \\
\text { Index }\end{array}$ & $\begin{array}{c}\text { Parent } \\
\text { Index }\end{array}$ & $\begin{array}{c}\text { Daughter } \\
\text { Index }\end{array}$ & $\begin{array}{c}\text { Parent } \\
\text { Index }\end{array}$ & $\begin{array}{c}\text { Daughter } \\
\text { Index }\end{array}$ \\
\hline 3 & 2 & 47 & 46 & 96 & 91 \\
\hline 4 & 3 & 48 & 46 & 97 & 84 \\
\hline 5 & 3 & 49 & 48 & 98 & 84 \\
\hline 6 & 2 & 50 & 48 & 99 & 97 \\
\hline 7 & 6 & 51 & 45 & 100 & 97 \\
\hline 8 & 6 & 51 & 50 & 101 & 95 \\
\hline 9 & 7 & 54 & 53 & 102 & 95 \\
\hline 10 & 9 & 55 & 53 & 103 & 98 \\
\hline 11 & 9 & 57 & 45 & 104 & 98 \\
\hline 12 & 7 & 57 & 55 & 109 & 108 \\
\hline 14 & 13 & 57 & 56 & 109 & 112 \\
\hline 15 & 14 & 61 & 60 & 111 & 110 \\
\hline 16 & 14 & 62 & 60 & 112 & 111 \\
\hline 17 & 16 & 63 & 62 & 113 & 109 \\
\hline 18 & 16 & 64 & 62 & 115 & 114 \\
\hline 19 & 18 & 66 & 65 & 161 & 115 \\
\hline 20 & 18 & 67 & 65 & 116 & 115 \\
\hline 21 & 13 & 68 & 61 & 117 & 116 \\
\hline 22 & 21 & 69 & 61 & 118 & 111 \\
\hline 23 & 21 & 70 & 69 & 119 & 117 \\
\hline 24 & 23 & 71 & 70 & 119 & 120 \\
\hline 25 & 23 & 73 & 72 & 120 & 170 \\
\hline 27 & 26 & 74 & 73 & 120 & 175 \\
\hline 28 & 27 & 75 & 73 & 121 & 152 \\
\hline 29 & 27 & 76 & 73 & 122 & 121 \\
\hline 30 & 28 & 77 & 72 & 123 & 104 \\
\hline 31 & 28 & 78 & 69 & 124 & 121 \\
\hline 32 & 26 & 79 & 67 & 125 & 119 \\
\hline 33 & 32 & 80 & 67 & 126 & 119 \\
\hline 34 & 33 & 81 & 77 & 127 & 156 \\
\hline 35 & 33 & 82 & 80 & 128 & 127 \\
\hline 36 & 35 & 83 & 80 & 129 & 128 \\
\hline 37 & 35 & 87 & 86 & 130 & 127 \\
\hline 38 & 37 & 89 & 87 & 131 & 156 \\
\hline 39 & 37 & 89 & 88 & 132 & 131 \\
\hline 40 & 32 & 91 & 90 & 133 & 128 \\
\hline 41 & 40 & 92 & 90 & 134 & 96 \\
\hline 42 & 40 & 94 & 93 & 135 & 134 \\
\hline 43 & 42 & 95 & 93 & 136 & 96 \\
\hline 44 & 42 & 96 & 89 & 137 & 104 \\
\hline
\end{tabular}




\begin{tabular}{|c|c|c|c|c|c|}
\hline $\begin{array}{l}\text { Parent } \\
\text { Index }\end{array}$ & $\begin{array}{c}\text { Daughter } \\
\text { Index }\end{array}$ & $\begin{array}{c}\text { Parent } \\
\text { Index }\end{array}$ & $\begin{array}{c}\text { Daughter } \\
\text { Index }\end{array}$ & $\begin{array}{c}\text { Parent } \\
\text { Index }\end{array}$ & $\begin{array}{c}\text { Daughter } \\
\text { Index }\end{array}$ \\
\hline 137 & 136 & 173 & 159 & 219 & 217 \\
\hline 138 & 104 & 174 & 171 & 220 & 202 \\
\hline 138 & 136 & 174 & 172 & 221 & 202 \\
\hline 139 & 104 & 174 & 178 & 222 & 221 \\
\hline 140 & 134 & 175 & 173 & 223 & 221 \\
\hline 141 & 140 & 175 & 174 & 224 & 209 \\
\hline 142 & 140 & 176 & 162 & 225 & 209 \\
\hline 143 & 133 & 177 & 59 & 226 & 209 \\
\hline 143 & 135 & 178 & 166 & 227 & 206 \\
\hline 144 & 139 & 179 & 173 & 228 & 206 \\
\hline 145 & 109 & 179 & 174 & 230 & 229 \\
\hline 146 & 139 & 180 & 157 & 234 & 229 \\
\hline 147 & 122 & 183 & 182 & 235 & 230 \\
\hline 148 & 122 & 184 & 182 & 236 & 232 \\
\hline 149 & 131 & 188 & 187 & 237 & 232 \\
\hline 150 & 144 & 189 & 187 & 239 & 238 \\
\hline 151 & 144 & 191 & 190 & 240 & 238 \\
\hline 152 & 158 & 192 & 190 & 242 & 241 \\
\hline 153 & 158 & 193 & 192 & 243 & 241 \\
\hline 154 & 79 & 194 & 192 & 245 & 244 \\
\hline 155 & 81 & 195 & 192 & 247 & 244 \\
\hline 156 & 154 & 196 & 191 & 249 & 225 \\
\hline 156 & 155 & 197 & 196 & 250 & 249 \\
\hline 157 & 83 & 198 & 196 & 251 & 250 \\
\hline 158 & 157 & 199 & 191 & 252 & 250 \\
\hline 159 & 57 & 201 & 200 & 253 & 223 \\
\hline 162 & 77 & 202 & 201 & 257 & 255 \\
\hline 163 & 122 & 203 & 200 & 258 & 256 \\
\hline 164 & 163 & 204 & 203 & 259 & 255 \\
\hline 165 & 163 & 205 & 203 & 260 & 259 \\
\hline 166 & 78 & 206 & 205 & 261 & 259 \\
\hline 167 & 78 & 207 & 205 & 262 & 256 \\
\hline 168 & 160 & 208 & 204 & 265 & 263 \\
\hline 169 & 114 & 209 & 204 & 265 & 264 \\
\hline 161 & 169 & 213 & 212 & 267 & 266 \\
\hline 170 & 168 & 214 & 212 & 268 & 266 \\
\hline 170 & 169 & 215 & 214 & 270 & 271 \\
\hline 171 & 51 & 216 & 214 & 271 & 269 \\
\hline 172 & 57 & 217 & 201 & 272 & 271 \\
\hline 173 & 85 & 218 & 217 & 273 & 271 \\
\hline
\end{tabular}




\begin{tabular}{|c|c|c|c|c|c|}
\hline $\begin{array}{l}\text { Parent } \\
\text { Index }\end{array}$ & $\begin{array}{c}\text { Daughter } \\
\text { Index }\end{array}$ & $\begin{array}{c}\text { Parent } \\
\text { Index }\end{array}$ & $\begin{array}{c}\text { Daughter } \\
\text { Index }\end{array}$ & $\begin{array}{c}\text { Parent } \\
\text { Index }\end{array}$ & $\begin{array}{c}\text { Daughter } \\
\text { Index }\end{array}$ \\
\hline 274 & 270 & 319 & 315 & 360 & 348 \\
\hline 275 & 274 & 320 & 315 & 360 & 351 \\
\hline 276 & 274 & 321 & 320 & 361 & 345 \\
\hline 277 & 270 & 322 & 320 & 362 & 348 \\
\hline 278 & 277 & 323 & 321 & 362 & 351 \\
\hline 279 & 278 & 324 & 321 & 363 & 322 \\
\hline 280 & 278 & 325 & 324 & 364 & 322 \\
\hline 281 & 277 & 326 & 325 & 365 & 363 \\
\hline 283 & 282 & 327 & 326 & 366 & 363 \\
\hline 284 & 283 & 328 & 325 & 367 & 362 \\
\hline 285 & 283 & 329 & 328 & 368 & 362 \\
\hline 288 & 286 & 330 & 326 & 369 & 361 \\
\hline 288 & 287 & 331 & 324 & 370 & 361 \\
\hline 291 & 289 & 332 & 331 & 371 & 368 \\
\hline 291 & 290 & 333 & 332 & 372 & 370 \\
\hline 292 & 291 & 334 & 333 & 372 & 371 \\
\hline 293 & 288 & 335 & 333 & 373 & 360 \\
\hline 293 & 292 & 336 & 335 & 374 & 364 \\
\hline 294 & 293 & 337 & 335 & 375 & 364 \\
\hline 295 & 293 & 338 & 332 & 376 & 360 \\
\hline 296 & 282 & 339 & 338 & 378 & 377 \\
\hline 297 & 296 & 340 & 338 & 379 & 377 \\
\hline 298 & 296 & 342 & 341 & 381 & 380 \\
\hline 299 & 291 & 343 & 342 & 382 & 380 \\
\hline 302 & 301 & 344 & 342 & 383 & 382 \\
\hline 303 & 301 & 345 & 344 & 384 & 382 \\
\hline 304 & 303 & 346 & 341 & 385 & 384 \\
\hline 305 & 304 & 347 & 344 & 386 & 384 \\
\hline 306 & 304 & 348 & 347 & 387 & 300 \\
\hline 307 & 305 & 349 & 347 & 388 & 300 \\
\hline 308 & 303 & 350 & 349 & 393 & 392 \\
\hline 309 & 305 & 351 & 349 & 394 & 392 \\
\hline 310 & 308 & 352 & 345 & 395 & 383 \\
\hline 312 & 311 & 353 & 352 & 396 & 383 \\
\hline 313 & 312 & 354 & 352 & 397 & 396 \\
\hline 314 & 312 & 355 & 354 & 398 & 396 \\
\hline 315 & 313 & 356 & 354 & 399 & 398 \\
\hline 316 & 313 & 357 & 331 & 400 & 398 \\
\hline 317 & 316 & 358 & 357 & 401 & 366 \\
\hline 318 & 316 & 359 & 357 & 402 & 366 \\
\hline
\end{tabular}




\begin{tabular}{|c|c|c|c|}
\hline $\begin{array}{l}\text { Parent } \\
\text { Index }\end{array}$ & $\begin{array}{c}\text { Daughter } \\
\text { Index }\end{array}$ & \multirow{11}{*}{$\begin{array}{l}\text { Parent } \\
\text { Index }\end{array}$} & \multirow{11}{*}{$\begin{array}{c}\text { Daughte } \\
\text { Index }\end{array}$} \\
\hline 403 & 356 & & \\
\hline 404 & 402 & & \\
\hline 405 & 374 & & \\
\hline 405 & 375 & & \\
\hline 406 & 401 & & \\
\hline 407 & 401 & & \\
\hline 408 & 358 & & \\
\hline 409 & 340 & & \\
\hline 409 & 406 & & \\
\hline 410 & 356 & & \\
\hline 711 & 000 & 58 & 258 \\
\hline 412 & 358 & 56 & 261 \\
\hline 413 & 402 & 85 & 265 \\
\hline 414 & 403 & 114 & 267 \\
\hline 415 & 337 & 110 & 267 \\
\hline 415 & 414 & 241 & 346 \\
\hline 416 & 402 & 107 & 334 \\
\hline 417 & 416 & 106 & 310 \\
\hline 418 & 409 & 160 & 267 \\
\hline 419 & 337 & 88 & 252 \\
\hline 419 & 414 & 105 & 251 \\
\hline 420 & 408 & 90 & 253 \\
\hline 420 & 410 & 86 & 228 \\
\hline 121 & 410 & 391 & 220 \\
\hline 721 & T10 & 93 & 231 \\
\hline 40 & 104 & 108 & 284 \\
\hline 46 & 185 & 110 & 279 \\
\hline 52 & 186 & 248 & 416 \\
\hline 59 & 189 & 246 & 371 \\
\hline 60 & 188 & & \\
\hline 65 & 188 & & \\
\hline 72 & 199 & & \\
\hline 84 & 181 & & \\
\hline 238 & 391 & & \\
\hline 229 & 393 & & \\
\hline 244 & 318 & & \\
\hline 232 & 397 & & \\
\hline 233 & 399 & & \\
\hline 231 & 390 & & \\
\hline 53 & 258 & & \\
\hline
\end{tabular}




\section{Bibliography}

[1] E. Rahbar, J. Weimer, H. Gibbs, A. T. Yeh, C. D. Bertram, M. J. Davis, M. A. Hill, D. C. Zawieja, and J. E. Moore Jr., "Passive pressure-diameter relationship and structural composition of rat mesenteric lymphangions," Lymphat Res Biol, vol. 10, no. 4, pp. 152-63, 2012.

[2] M. J. Davis, E. Rahbar, A. A. Gashev, D. C. Zawieja, and J. E. Moore Jr., "Determinants of valve gating in collecting lymphatic vessels from rat mesentery," Am J Physiol Heart Circ Physiol, vol. 301, no. 1, pp. H48-60, 2011.

[3] N. G. McHale and I. C. Roddie, "The effect of transmural pressure on pumping activity in isolated bovine lymphatic vessels.," J. Physiol, vol. 261, pp. 255-269, 1976.

[4] M. P. C. Sappey, Anatomie, Physiologie, Pathologie des vaisseaux lymphatiques considérés ches l’homme et les vertébrés. Paris: Biusanté, 1834.

[5] J. Seki, Y. Satomura, and Y. Ooi, "Velocity pulse advances pressure pulse by close to 45 degrees in the rat pial arterioles.," Biorheology, vol. 41, 2004.

[6] M. Meyer, M. Desbrun, P. Schrader, and A. H. Barr, Discrete DifferentialGeometry Operators for Triangulated 2-Manifolds, pp. 35-57. Mathematics and Visualization, Springer Berlin Heidelberg, 2003.

[7] A. Cheville, C. McGarvey, J. Petrek, S. Russo, S. Thiadens, and M. Taylor, "The grading of lymphedema in oncology clinical trials," Seminars in Radiation Oncology, vol. 13, no. 3, pp. 214-225, 2003. 
[8] B. J. Smoot, J. F. Wong, and M. J. Dodd, "Comparison of diagnostic accuracy of clinical measures of breast cancer-related lymphedema: area under the curve," Arch Phys Med Rehabil, vol. 92, no. 4, pp. 603-10, 2011.

[9] M. Potente and T. Makinen, "Vascular heterogeneity and specialization in development and disease," Nat Rev Mol Cell Biol, vol. 18, no. 8, pp. 477-494, 2017.

[10] G. S. István Rusznyák, Mihály Földi, Lymphatics and Lymph Circulation. Pergamon Press, 1967.

[11] C. C. Clement, W. Wang, M. Dzieciatkowska, M. Cortese, K. C. Hansen, A. Becerra, S. Thangaswamy, I. Nizamutdinova, J. Y. Moon, L. J. Stern, A. A. Gashev, D. Zawieja, and L. Santambrogio, "Quantitative profiling of the lymph node clearance capacity," Sci Rep, vol. 8, no. 1, p. 11253, 2018.

[12] M. A. Swartz, "Immunomodulatory roles of lymphatic vessels in cancer progression," Cancer Immunol Res, vol. 2, no. 8, pp. 701-7, 2014.

[13] K. Alitalo, "The lymphatic vasculature in disease," Nat Med, vol. 17, no. 11, pp. 1371-80, 2011.

[14] M. A. Swartz, J. A. Hubbell, and S. T. Reddy, "Lymphatic drainage function and its immunological implications: from dendritic cell homing to vaccine design," Semin Immunol, vol. 20, no. 2, pp. 147-56, 2008.

[15] R. Carola, C. P. Noback, and J. P. Hartley, Human Anatomy and Physiology. New York: McGraw-Hill, international ed., 1990.

[16] M. Permanyer, B. Bosnjak, and R. Forster, "Dendritic cells, t cells and lymphatics: dialogues in migration and beyond," Curr Opin Immunol, vol. 53, pp. 173-179, 2018.

[17] L. E. Wineski and R. S. Snell, Snell's Clinical Anatomy by Regions. Philadelphia: Wolters Kluwer, 9 ed., 2018. 
[18] O. Catalano, A. Nunziata, P. P. Saturnino, and A. Siani, "Epitrochlear lymph nodes: Anatomy, clinical aspects, and sonography features. pictorial essay(), $J$ Ultrasound, vol. 13, no. 4, pp. 168-74, 2010.

[19] M. A. Aydin, B. Okudan, S. Nasir, M. Ozbek, A. Karagoz, and S. Altuntas, "Lymphoscintigraphic drainage of acral limb skin to interval sentinel lymph nodes in healthy subjects," J Surg Oncol, vol. 93, no. 4, pp. 286-93, 2006.

[20] S. Roberto, B. Valeria, V. Roberto del, M. Raffaella, F. A. Chiara, and R. Leopoldo, "Analysis by high resolution ultrasound of superficial lymph nodes: anatomical, morphological and structural variations," Clin Imaging, vol. 38, no. 2, pp. $96-9,2014$.

[21] S. K. Bains, J. Ballinger, S. Allen, A. W. Stanton, J. R. Levick, P. S. Mortimer, A. D. Purushotham, and A. M. Peters, "An investigation of lymphovenous communications in the upper limbs of breast cancer patients," Eur J Surg Oncol, vol. 41, no. 3, pp. 433-8, 2015.

[22] G. Natale, G. Bocci, and D. Ribatti, "Scholars and scientists in the history of the lymphatic system," J Anat, vol. 231, no. 3, pp. 417-429, 2017.

[23] H. Florey, "Observations on the contractility of lacteals," J Physiol, 1928.

[24] L. Ranvier, "The développement des vaisseaux lymphatiques," C. R. Acad. Sci., vol. 121, pp. 1105-1109, 1895.

[25] O. F. Kampmeier, "The genetic history of the valve in the lymphatic system of man," American Journal of Anatomy, vol. 40, no. 3, pp. 413-457, 1928.

[26] K. Aukland, "Arnold heller and the lymph pump," Acta Physiol Scand, vol. 185, pp. 171-180, 2005.

[27] J. Sheldon, On the Absorbent System. 1784.

[28] R. O. Smith, "Lymphatic contracility: A possible intrinsic mechanism of lymphatic vessels for the transport of lymph," J Exp Med, vol. 90, pp. 497-509, 1949. 
[29] D. A. Zhdanov, "Sanitarno-gigienicheskogo,"

[30] H. J. D. Mawhinney and I. Roddie, "Spontaneous activity in isolated bovine mesenteric lymphatics," J. Physiol, vol. 229, pp. 339-345, 1973.

[31] T. Ohhashi, T. Azuma, and M. Sakaguchi, "Active and passive mechanical characteristics," Am. J. Physiol, vol. 239, pp. 88-95, 1980.

[32] N. P. Reddy, T. A. Krouskop, and P. H. Newell, "A computer model of the lymphatic system," Comput. Biol. Med, vol. 7, pp. 181-197, 1976.

[33] C. M. Quick, A. M. Venugopal, A. A. Gashev, D. C. Zawieja, and R. H. Stewart, "Intrinsic pump-conduit behavior of lymphangions," Am J Physiol Regul Integr Comp Physiol, vol. 292, no. 4, pp. R1510-8, 2007.

[34] A. J. Macdonald, K. P. Arkill, G. R. Tabor, N. G. McHale, and C. P. Winlove, "Modeling flow in collecting lymphatic vessels: one-dimensional flow through a series of contractile elements," Am J Physiol Heart Circ Physiol, vol. 295, no. 1, pp. H305-13, 2008.

[35] C. Contarino and E. F. Toro, "A one-dimensional mathematical model of collecting lymphatics coupled with an electro-fluid-mechanical contraction model and valve dynamics," Biomech Model Mechanobiol, vol. 17, no. 6, pp. 1687-1714, 2018.

[36] K. P. Arkill, J. Moger, and C. P. Winlove, "The structure and mechanical properties of collecting lymphatic vessels: an investigation using multimodal nonlinear microscopy," Journal of Anatomy, vol. 216, pp. 547-555, 2010.

[37] A. W. Caulk, Z. V. Nepiyushchikh, R. Shaw, J. B. Dixon, and J. Gleason, R. L., "Quantification of the passive and active biaxial mechanical behaviour and microstructural organization of rat thoracic ducts," $J R$ Soc Interface, vol. 12, no. 108, p. 20150280, 2015.

[38] T. Ohhashi, "Comparison of viscoelastic properties of walls and functional characteristics of valves in lymphatic and venous vessels.," Lymphology, vol. 20, pp. 219223, 1987. 
[39] E. Rahbar and J. E. Moore Jr., "A model of a radially expanding and contracting lymphangion," J Biomech, vol. 44, no. 6, pp. 1001-7, 2011.

[40] C. D. Bertram, C. Macaskill, M. J. Davis, and J. E. Moore Jr., "Development of a model of a multi-lymphangion lymphatic vessel incorporating realistic and measured parameter values," Biomechanics and Modeling in Mechanobiology, vol. 13, no. 2, pp. 401-16, 2014.

[41] C. D. Bertram, C. Macaskill, and J. E. Moore Jr., "Pump function curve shape for a model lymphatic vessel," Med Eng Phys, vol. 38, no. 7, pp. 656-663, 2016.

[42] S. Jamalian, C. D. Bertram, W. J. Richardson, and J. E. Moore Jr., "Parameter sensitivity analysis of a lumped-parameter model of a chain of lymphangions in series," Am J Physiol Heart Circ Physiol, vol. 305, no. 12, pp. H1709-17, 2013.

[43] S. Jamalian, M. J. Davis, D. C. Zawieja, and J. E. Moore Jr., "Network scale modeling of lymph transport and its effective pumping parameters," PLoS One, vol. 11, no. 2, p. e0148384, 2016.

[44] S. Jamalian, M. Jafarnejad, S. D. Zawieja, C. D. Bertram, A. A. Gashev, D. C. Zawieja, M. J. Davis, and J. E. Moore Jr, "Demonstration and analysis of the suction effect for pumping lymph from tissue beds at subatmospheric pressure," Sci Rep, vol. 7, no. 1, p. 12080, 2017.

[45] M. Takada, "The ultrastructure of lymphatic valves in rabbits and mice," $A m J$ Anat, vol. 132, pp. 207-218, 1971.

[46] J. Vajda and M. Tomcsik, "The structure of the valves of the lymphatic vessels.," Acta Anat, vol. 78, pp. 521-531, 1971.

[47] M. C. Mazzoni, T. C. Skalak, and G. W. Schmid-Schönbein, "Structure of lymphatic valves in the spinotrapezium muscle of the rat," Blood Vessels, vol. 24, pp. 304-312, 1987.

[48] C. Bellini and R. C. Hennekam, Clinical Disorders of Primary Malfunctioning of the Lymphatic System, pp. 187-204. Vienna: Springer Vienna, 2014. 
[49] J. T. Wilson, W. Wang, A. H. Hellerstedt, D. C. Zawieja, and J. E. Moore Jr., "Confocal image-based computational modeling of nitric oxide transport in a rat mesenteric lymphatic vessel," J Biomech Eng, vol. 135, no. 5, p. 51005, 2013.

[50] K. N. Margaris and R. A. Black, "Modelling the lymphatic system: challenges and opportunities," J R Soc Interface, vol. 9, no. 69, pp. 601-12, 2012.

[51] J. T. Wilson, R. van Loon, W. Wang, D. C. Zawieja, and J. E. Moore Jr., "Determining the combined effect of the lymphatic valve leaflets and sinus on resistance to forward flow," J Biomech, vol. 48, no. 13, pp. 3584-90, 2015.

[52] A. Macdonald, The Computational Modelling of Collecting Lymphatic Vessels. PhD thesis, 2008.

[53] J. T. Wilson, T. L. Edgar, S. Prabhakar, M. Horner, R. van Loon, and J. E. Moore Jr., "A fully coupled fluid-structure interaction model of the secondary lymphatic valve," Computer Methods in Biomechanics and Biomedical Engineering, 2018.

[54] C. D. Bertram, C. Macaskill, and J. E. Moore Jr., "Simulation of a chain of collapsible contracting lymphangions with progressive valve closure," J Biomech Eng, vol. 133, no. 1, p. 011008, 2011.

[55] J. M. Carson, Development of a cardiovascular and lymphatic network model during pregnancy. Thesis, 2018.

[56] W. Yao, S.-Z. Wang, G.-H. Ding, and X.-Y. Shen, "A linear dynamic model describing lymph circulation," Journal of Hydrodynamics, vol. 21, no. 1, pp. 118-123, 2009.

[57] L. L. Munn, "Mechanobiology of lymphatic contractions," Semin Cell Dev Biol, vol. 38, pp. 67-74, 2015.

[58] R. Tretyakova, R. Savinkov, G. Lobov, and G. Bocharov, "Developing computational geometry and network graph models of human lymphatic system," Computation, vol. 6, no. 1, 2017. 
[59] H. Suami, G. I. Taylor, and W. R. Pan, "The lymphatic territories of the upper limb: anatomical study and clinical implications," Plast Reconstr Surg, vol. 119, no. 6, pp. 1813-22, 2007.

[60] S. Strandring, N. R. Borley, and H. Gray, Gray's Anatomy: the anatomical basis of clinical practice. Edinburgh: Churchill Livingstone, 2008.

[61] J. C. Rasmussen, S. Kwon, E. M. Sevick-Muraca, and J. N. Cormier, "The role of lymphatics in cancer as assessed by near-infrared fluorescence imaging," Ann Biomed Eng, vol. 40, no. 2, pp. 408-21, 2012.

[62] P. S. Mortimer, "Therapy approaches for lymphedema," Angiology, vol. 48, no. 1, pp. 87-91, 1997.

[63] C. J. Chang and J. N. Cormier, "Lymphedema interventions: exercise, surgery, and compression devices," Semin Oncol Nurs, vol. 29, no. 1, pp. 28-40, 2013.

[64] A. L. Moseley, C. J. Carati, and N. B. Piller, "A systematic review of common conservative therapies for arm lymphoedema secondary to breast cancer treatment," Ann Oncol, vol. 18, no. 4, pp. 639-46, 2007.

[65] F. Pianosi, F. Sarrazin, and T. Wagener, "A matlab toolbox for global sensitivity analysis,," Enviromental Modelling \& Software, 2015.

[66] G. E. B. Archer, A. Saltelli, and I. M. Sobol, "Sensitivity measures,anova-like techniques and the use of bootstrap," Journal of Statistical Computation and Simulation, vol. 58, no. 2, pp. 99-120, 1997.

[67] A. Saltelli, P. Annoni, I. Azzini, F. Campolongo, M. Ratto, and S. Tarantola, "Variance based sensitivity analysis of model output. design and estimator for the total sensitivity index," Computer Physics Communications, vol. 181, no. 2, pp. 259-270, 2010.

[68] S. Pant, C. Corsini, C. Baker, T. Y. Hsia, G. Pennati, and I. E. Vignon-Clementel, "Inverse problems in reduced order models of cardiovascular haemodynamics: aspects of data assimilation and heart rate variability," $J R$ Soc Interface, vol. 14, no. $126,2017$. 
[69] R. F. Uren, R. Howman-Giles, and J. F. Thompson, "Patterns of lymphatic drainage from the skin in patients with melanoma," J Nucl Med, vol. 44, pp. 570$582,2003$.

[70] J. C. Rasmussen, I. C. Tan, M. V. Marshall, K. E. Adams, S. Kwon, C. E. Fife, E. A. Maus, L. A. Smith, K. R. Covington, and E. M. Sevick-Muraca, "Human lymphatic architecture and dynamic transport imaged using near-infrared fluorescence," Transl Oncol, vol. 3, no. 6, pp. 362-72, 2010.

[71] N. Leung, D. Furniss, and H. Giele, "Modern surgical management of breast cancer therapy related upper limb and breast lymphoedema," Maturitas, vol. 80, no. 4, pp. $384-90,2015$.

[72] A. G. Warren, H. Brorson, L. J. Borud, and S. A. Slavin, "Lymphedema: a comprehensive review," Ann Plast Surg, vol. 59, no. 4, pp. 464-72, 2007.

[73] B. Lee, M. Andrade, J. Bergan, F. Boccardo, C. Campisi, R. Damstra, M. Flour, P. Gloviczki, J. Laredo, N. Piller, S. Michelini, P. Mortimer, and J. Villavicencio, "Diagnosis and treatment of primary lymphedema. consensus document of the international union of phlebology(iup)," International Angiology, 2009.

[74] "The diagnosis and treatment of peripheral lymphedema: 2016 consensus document of the international society of lymphology," report, The Executive Committee of the International Society of Lymphology, 2016.

[75] M. Bernas, "Assessment and risk reduction in lymphedema," Semin Oncol Nurs, vol. 29, no. 1, pp. 12-9, 2013.

[76] M. Mihara, H. Hara, J. Araki, K. Kikuchi, M. Narushima, T. Yamamoto, T. Iida, H. Yoshimatsu, N. Murai, K. Mitsui, T. Okitsu, and I. Koshima, "Indocyanine green (icg) lymphography is superior to lymphoscintigraphy for diagnostic imaging of early lymphedema of the upper limbs," PLoS One, vol. 7, no. 6, p. e38182, 2012. 
[77] S. Akita, R. Nakamura, N. Yamamoto, H. Tokumoto, T. Ishigaki, Y. Yamaji, Y. Sasahara, Y. Kubota, N. Mitsukawa, and K. Satoh, "Early detection of lymphatic disorder and treatment for lymphedema following breast cancer," Plast Reconstr Surg, vol. 138, no. 2, pp. 192e-202e, 2016.

[78] J. M. Armer, "The problem of post-breast cancer lymphedema: impact and measurement issues," Cancer Invest, vol. 23, no. 1, pp. 76-83, 2005.

[79] J. P. Belgrado, P. Bracale, J. Bates, N. Rö, R. Rosiello, A. Cangiano, and J. Moraine, "Lymphoedema: What can be measured and how: Overview," The European Journal of Lymphology, vol. 21, 2010.

[80] J. A. Petrek, R. T. Senie, M. Peters, and P. P. Rosen, "Lymphedema in a cohort of breast carcinoma survivors 20 years after diagnosis," American Cancer Society, vol. 92, no. 6, pp. 1368-1377, 2001.

[81] L. Gerber, M. Lampert, C. Wood, M. Duncan, T. D'angelo, W. Schain, H. McDonald, D. Danforth, P. Findlay, E. Glatstein, M. E. Lippman, S. M. Steinberg, C. Gorrell, A. Lichter, and E. Demoss, "Comparison of pain, motion and edema after modified radical mastectomy vs. local excision with axillary dissection and radiation," Breast Cancer Research and Treatment, vol. 21, pp. 139-145, 1992.

[82] N. L. Stout Gergich, L. A. Pfalzer, C. McGarvey, B. Springer, L. H. Gerber, and P. Soballe, "Preoperative assessment enables the early diagnosis and successful treatment of lymphedema," Cancer, vol. 112, no. 12, pp. 2809-19, 2008.

[83] K. Segerström, P. Bjerle, and Å. Nyström, "Importance of time in assessing arm and hand function after treatment of breast cancer," Scandinavian Journal of Plastic and Reconstructive Surgery and Hand Surgery, vol. 25, no. 3, pp. 241-244, 1991.

[84] P. Schrenk, R. Reiger, A. Shamiyeh, and W. Wayland, "Morbidity following sentinel lymph node biopsy versus axillary lymph node dissection for patients with breast carcinoma," American Cancer Society, vol. 88, pp. 608-614, 1998. 
[85] G. Liljegren and L. Holmberg, "Arm morbidity after sector resection and axillary dissection with or without postoperative radiotherapy in breast cancer stage i. results from a randomised trail," European Journal of Cancer, vol. 33, no. 2, pp. 193-199, 1997.

[86] K. D. Kiel and A. W. Rademacker, "Early-stage breast cancer: Arm edema after wide excision and breast irradiation," Therapeutic Radiology, vol. 198, pp. 279$283,1996$.

[87] A. Keramopoulos, C. Rsionou, D. Minaretzis, S. Michalas, and D. Aravantinos, "Arm morbidity following treatment of breast cancer with total axillary dissection: A multivariated approach," Oncology, vol. 50, pp. 445-449, 1993.

[88] R. S. Werner, B. McCormick, J. Petrek, L. Cox, C. Cirrincione, J. R. Gray, and J. Yahalom, "Arm edema in conservatively managed breast cancer: Obesity is a major predicitve factor," Theraputic Radiology, vol. 180, pp. 177-184, 1991.

[89] P. P. Lin, D. C. Allison, J. Wainstock, K. D. Miller, W. C. Dooley, N. Friedman, and R. R. Baker, "Impact of axillary lymph node dissection on the therapy of breast cancer patients," Journal of Clinical Oncology, vol. 11, no. 8, pp. 1536$1544,1993$.

[90] G. K. Sylvia M. Ramos, Linda S. O'Donnell, "Edema volume, not timing, is the key to success in lymphedema treatment," American Journal of Surgery, vol. 178, p. 4, 1999 .

[91] J. R. Casley-Smith, "Alterations of untreated lymphedema and its grades over time," Lymphology, vol. 28, 1995.

[92] N. Adriaenssens, R. Buyl, P. Lievens, C. Fontaine, and J. Lamote, "Comparative study between mobile infrared optoelectronic volumetry with a perometer and two commonly used methods for the evaluation of arm volume in patients with breast cancer related lymphedema of the arm.," Lymphology, vol. 46, 2013.

[93] B. Cornish, L. Ward, B. Thomas, and I. Bunce, "Quantification of lymphoedema using multi-frequency bioimpedance," Appl. Radiat. Isop., vol. 49, p. 2, 1998. 
[94] K. Karakashian, L. Shaban, C. Pike, and R. van Loon, "Investigation of shape with patients suffering from unilateral lymphoedema," Ann Biomed Eng, vol. 46, no. 1, pp. 108-121, 2018.

[95] K. C. Johnson, A. G. Kennedy, and S. M. Henry, "Clinical measurements of lymphedema," Lymphat Res Biol, vol. 12, no. 4, pp. 216-21, 2014.

[96] M. Reuter, S. Biasotti, D. Giorgi, G. Patanéš, and M. Spagnuolo, "Discrete laplacebeltrami operators for shape analysis and segmentation," Computers $\&$ Graphics, vol. 33, no. 3, pp. 381-390, 2009.

[97] B. Levy, "Laplace-beltrami eigenfunctions towards an algorithm that understands geometry," in IEEE International Conference on Shape Modeling and Applications, pp. 13-26.

[98] A. J. Chaudhari, R. M. Leahy, B. L. Wise, N. E. Lane, R. D. Badawi, and A. A. Joshi, "Global point signature for shape analysis of carpal bones," Phys Med Biol, vol. 59, no. 4, pp. 961-73, 2014.

[99] M. Wardetzky, S. Mathur, F. Kälberer, and E. Grinspun, "Discrete laplace operators: No free lunch," in Eurographics Symposium on Geometry Processing (A. Belyaev and M. Garland, eds.).

[100] W. N. P. Martin, Reuter; Franz-Erich, "Laplace-spectra as fingerprints for shape matching," Proceedings of the 2005 ACM conference, 2005.

[101] R. M. Rustamov, "Laplace-beltrami eigenfunctions for deformation invariant shape representation," Eurographics Symposium on Geometry Processing, 2007.

[102] D. G. Duffy, Green's Functions with Applications, book section What Is A Green's Function, p. 446. St. Louis, Washington: Chapman \& Hall, 2001.

[103] M. M. Bronstein and A. M. Bronstein, "Shape recognition with spectral distances," IEEE Trans Pattern Anal Mach Intell, vol. 33, no. 5, pp. 1065-71, 2011.

[104] C. E. Shannon and W. Weaver, The Mathematical Theory of Communication. Urbana: The University of Illinois Press, 1949. 
[105] J. T. Livarinen, R. K. Korhonen, P. Julkunen, and J. S. Jurvelin, "Experimental and computational analysis of soft tissue mechanical response under negative pressure in forearm," Skin Res Technol, vol. 19, no. 1, pp. e356-65, 2013.

[106] J. M. Godoy, S. H. Silva, and M. F. Godoy, "Sensitivity and specificity of combined perimetric and volumetric evaluations in the diagnosis of arm lymphedema," Prague Med Rep, vol. 108, no. 3, pp. 243-7, 2007. 